\title{
1 Mobilization of antibiotic resistance genes differ by resistance mechanism
}

2 Tue Kjærgaard Nielsen ${ }^{1 *}$, Patrick Denis Browne ${ }^{1}$, Lars Hestbjerg Hansen ${ }^{{ }^{*}}$

$3{ }^{1}$ Department of Plant and Environmental Sciences, University of Copenhagen, Thorvaldsensvej 40,

4 Frederiksberg C, 1871, Denmark

$5 \quad{ }^{*}$ Corresponding author e-mail: tkn@plen.ku.dk; Ihha@plen.ku.dk

List of abbreviations

$9 \quad$ ARG: Antibiotic Resistance Gene

10 MGE: Mobile Genetic Element

11 Tn: Transposon

12 IS: Insertion Sequence

13 AE: Antibiotic Efflux

14 Al: Antibiotic Inactivation

15 ATA: Antibiotic Target Alteration

16 ATR: Antibiotic Target Replacement

17 ATP: Antibiotic Target Protection

RPA: Reduced Permeability to Antibiotic

Abstract

The degree to which antibiotic resistance genes (ARGs) are mobilized by insertion sequence (IS) elements, plasmids, and integrons has a strong association with their likelihood to function as resistance determinants. This stems from genetic decontextualization where strong promoters often present in IS elements and integrons and the copy number effect of plasmids contribute to high expression of accessory genes. Here, we screen all complete bacterial RefSeq genomes for ARGs. The genetic contexts of detected ARGs are investigated for IS elements, integrons, plasmids, and phylogenetic dispersion. The ARG-MOB scale is proposed which indicates how mobilized detected ARGs are in bacterial genomes. Antibiotic efflux genes are rarely mobilized and it is concluded that these are often housekeeping genes that are not decontextualized to confer 
resistance through overexpression. Even $80 \%$ of $\beta$-lactamases have never, or very rarely, been mobilized in the 15,790 studied genomes. However, some ARGs are indeed mobilized and co-occur with IS elements, plasmids, and integrons. These results have consequences for the design and interpretation of studies screening for resistance determinants, as mobilized ARGs pose a more concrete risk to human health, especially under heterologous expression, than groups of ARGs that have only been shown to confer resistance in cloning experiments.

\section{Main}

\section{Resistance determinants and context}

Health and environmental challenges imposed by antibiotic resistance has sparked enormous research efforts into characterising genetic resistance determinants. Combined with broad availability of 2 nd and $3 r$ generation sequencing technologies, studying the presence and prevalence of putative ARGs in the environment has become popular in recent years.

Antibiotic resistance remains a significant global issue despite numerous studies into understanding spread of genes via mobile genetic elements (MGEs) and devising and implementing mitigation strategies. But, most classes of putative ARGs are intrinsic to bacterial genomes and can be considered housekeeping genes. These do not confer resistance until they become 'decontextualized' by mobilization ${ }^{1-3}$. Many putative ARGs have only been shown to provide resistance when cloned into cloning vectors with significant overexpression or with constitutive expression in mutants. Examples of mobilization events include (i) transfer from chromosomes to plasmids via transposons (Tn), (ii) integration with integrative conjugative elements (ICE), and (iii) capture by integrons as a gene cassette ${ }^{4}$. This aspect is often ignored in culture-independent studies using (meta)genomics and/or (q)PCR-based detection where resistance is rarely experimentally verified. Thus, screening the environment for ARGs may give impressions that "resistance is everywhere" or that widespread resistance predates the use of antibiotics ${ }^{5-7}$, although the native roles of found ARGs may not be related to antibiotic resistance ${ }^{1,2}$. 


\section{Native roles of ARGs}

When coupling (meta)genomic predictions with culturing-based detection of resistant strains, it is often found that the two approaches do not agree ${ }^{8-12}$, partially attributable to the fact that gene expression is rarely considered in these studies ${ }^{13}$. Screening a genome for resistance markers against an ARG database often results in copious false-positive resistance predictions ${ }^{10}$. This issue is most pronounced for efflux-related markers where the specificity of prediction has been reported to be only 0.12 . The balanced accuracy of resistance marker prediction against two widely used databases was only 0.52 and 0.66 , showing that finding ARGs in a genome does not necessarily equate phenotypic resistance ${ }^{14}$. Almost all ARGs likely have native roles unrelated to resistance to clinical concentrations of antibiotics ${ }^{1}$. Many antibiotics are natural secondary metabolites, occurring at clinically insignificant concentrations, that are involved in inter-cellular communication, regulation of metabolism, and other housekeeping functions ${ }^{15}$. Putative ARGs have been found in and cloned from susceptible bacteria ${ }^{8}$, where they are simply performing their original nonresistance roles. For instance, efflux pumps ${ }^{2,16}, \beta$-lactamases ${ }^{17,18}$ and lipid A modifying proteins $(\mathrm{MCR})^{19}$ are encoded by genes which have housekeeping functions but may be decontextualized to confer resistance.

Functional metagenomic approaches, essentially cloning fragmented DNA into expression vectors followed by screening for antibiotic resistance ${ }^{6,7,20,21}$, has led to the identification of many putative ARGs. Such genes are obviously decontextualized in the experiment and their native roles may not be related to resistance. This has resulted in a problematic dissemination of resistance-related annotations in gene databases. Thus, sequence homology is a poor proxy for resistance and cultureindependent techniques will often yield misleading results if genetic contexts of ARGs are not considered. With recent advances in long-read sequencing, high-quality metagenome-assembled genomes can be achieved ${ }^{22}$ and it is now possible to include consideration of the genetic context of ARGs.

\section{Classifying ARGs and their risk} The association between ARGs and MGEs have profound effects on phenotypic resistance $3,4,23-25$ and it has been argued, e.g. in the "RESCon" framework ${ }^{26}$, that multiple aspects should be included in risk assessment of $A R G s^{27,28}$. Here, we initiate the route to more accurate ARG predictions by 
categorizing associations between ARGs and MGEs in all completed RefSeq genomes. Decontextualization of ARGs is explored here by association with I) IS elements, II) plasmids, III) integrons, and IV) their dispersal across distinct genera.

\section{Results}

\section{Screening RefSeq genomes for ARGs and data processing}

The curated CARD database for ARGs was used to find putative ARGs in all completed bacterial genomes from the RefSeq database $(n=15,790) .12,170$ bp up- and downstream of predicted ARGs were analysed for IS elements and integrons, while the type of replicon (plasmid or chromosome) was also considered. For more details, see Methods and Supplementary Information (Supplementary Note 1). All databases are assumed to be heavily biased, especially towards humanassociated bacteria of which many almost identical genomes have been uploaded to RefSeq, leading to overrepresentation of these compared to e.g. environmental bacteria. In order to ameliorate these biases (Supplementary Note 3), highly similar genetic loci with predicted ARGs $(n=176,888)$ were clustered to 53,895 Clustered Resistance Loci (CRLs), representing 1,176 Antibiotic Resistance Ontology (ARO) terms from CARD (Fig. 1). This reduced the taxonomic Euclidean distance between CARD and RefSeq databases from 30.89 to 10.26, showing that many ARG loci in RefSeq are highly similar (Supplementary Note 4). Loci with efflux-associated ARGs were more compressed by clustering than all other types, indicating that these are more conserved and undergo fewer genetic rearrangements. The antibiotic efflux (AE) mechanism is the most abundant category and its CRL count is more than two times more numerous than the second-largest category, antibiotic inactivation (AI), although Al has over 3 times as many AROs as AE (Fig. 1).

As expected, loci in human-associated genera were especially compressed by clustering, showing that these are indeed overrepresented in the RefSeq database (Supplementary Fig. 8).

Four mobilization parameter ratios were explored for each CRL (Fig. 2): A) replicon type, B) IS element-association, C) integron-association, and D) dispersal of CRL across genera (Simpson diversity). All parameters were calculated on a scale of $0-1$, with 1 indicating that a CRL is highly associated with the given parameter. The mean of the four ratios is termed the ARG-MOB score and 
121 indicates how much genes of a given ARO are mobilized on a scale of $0-1$. This is described further 122 in later sections and Methods (Supplementary Fig. 9). Prophages in genomes are not explored for 123 ARGs, since these are not thought to be common vectors ${ }^{29}$. Neither are ICEs explored, although they 124 are likely important in resistance development ${ }^{30}$, since they are underexplored and may be difficult 125 to predict in genomes.

\section{Association of ARGs with IS elements and plasmids}

Major resistance mechanisms (non-hybrid) are associated with IS elements and plasmids in varying degrees (Fig. 3). Furthermore, individual mechanisms were associated with different families of IS elements (Supplementary Note 5). AE AROs generally have very low IS and Replicon ratios, which indicates that ARGs of this mechanism are rarely mobilized by either IS elements or plasmids. Only a handful of AE AROs have both high IS and high Replicon ratios, including ARO3002693 (transposonencoded cmIA1 chloramphenicol exporter), AR03003836 (qacH subunit of fluoroquinolone exporter), and ARO3000165 (tetracycline efflux pump tetA). Many AE AROs contain a large number of unique CRLs, as is also reflected by AE CRL count in Fig. 1. Therefore, AE ARGs are rarely associated with either IS elements or plasmids. Distances, in terms of nucleotides, between ARGs and IS elements are larger for AE AROs than for other mechanisms, indicating that efflux ARGs are more "loosely" associated with IS elements than other mechanisms (Mann-Whitney U-test (MWU) P < 0.01; Supplementary Note 6). Contrary to AE, the Al mechanism has many AROs that have been mobilized by both IS elements and plasmids, but also AROs that are hardly mobilized at all (Fig. 3). With some exceptions, antibiotic target alteration (ATA) AROs have low IS and Replicon ratios while also exhibiting a low number of unique CRLs, indicating that ATA CRLs are conserved and often not decontextualized. On the other hand, antibiotic target replacement (ATR) AROs are more mobilized by IS elements and plasmids (Fig. 3).

Putative ARGs are more decontextualized in clinically relevant genera (Supplementary Figs. 12-14). As expected from database biases (Supplementary Figs. 6-8), Proteobacteria harbour $88.18 \%$ of unclustered ARG loci (Supplementary Fig. 2) and Proteobacteria have a higher median IS ratio than Firmicutes, Actinobacteria, and Bacteriodetes (Supplementary Note 7; MWU test; $p<0.05$ ). Within Proteobacteria, multiple families with clinically relevant members, such as Enterobacteriaceae, are highly associated with ARGs mobilized by IS elements and plasmids. 
Phyla whose members are more associated with the environment, such as Actinobacteria and Bacteriodetes, have lower median IS ratios than Proteobacteria. Generally, diving into specific families and genera shows that ARGs in human-associated bacteria are more mobilized than in others (Supplementary Table 3) and persistent fixation of mobilized ARGs are likely a consequence of human interference with pathogenic bacteria ${ }^{23}$. This is described in more detail in Supplementary Note 7.

\section{Integron-association varies across ARG classes}

Under selective pressure for resistance, ARGs may be decontextualized into integrons, where a strong promoter confers overexpression of said ARGs, resulting in phenotypic resistance. Using IntegronFinder ${ }^{31}$ on CRL sequences, 3,723 ARGs were identified as gene cassettes in integrons or clusters of attC sites lacking integron-integrases (CALIN). The most abundant major mechanism was Al with 2,684 unique CRL occurrences. ATR and AE were found in association with integrons in 694 and 310 CRLs, respectively (Supplementary Fig. 15). Interestingly, canonical sulfonamide resistance genes associated with Tn402 Class 1 integrons ${ }^{32}$, sul1-4, were not the most frequent submechanism associated with integrons and was here found associated with integrons in only 123 unique CRLS out of 2,017 total sul1-4 CRLs. Trimethoprim resistance $d f r$ genes associated with Class 2 integrons and $\mathrm{Tn} 7$ transposon $^{32}$ were here found in high abundance in association with integrons. The most abundant submechanism was the antibiotic inactivation ANT( $\left.3^{\prime \prime}\right)$ category for nucleotidylylation of aminoglycosides at the hydroxyl group at position 3" with 1,009 CRLs associated with integrons. ANT genes are often found in association with integrons ${ }^{33}$ and subgroups of ANT genes are discussed further in Supplementary Note 8. OXA-9 and OXA-1 $\beta$-lactamases are found in integrons in 98.51\% and $63.90 \%$ of the 67 and 277 CRLs, respectively, emphasising that these ARGs are of concern (Supplementary Table 4).

From these observations, it is evident that screening an environment with PCR for e.g. sulfonamide resistance genes will result in prediction of many unmobilized genes that are not overexpressed through an integron promoter. On the other hand, OXA $\beta$-lactamases are often associated with integrons and thus likely to confer resistance, but nevertheless these results emphasize the importance of considering the genetic contexts of ARGs. 


\section{Mobilization assessment based on four parameters}

Inspired by previous work ${ }^{1}$, we calculated a mobilization scale for each ARO, termed the ARG-MOB scale, which is based on IS and plasmid ratios, integron-associations, and dispersal across genera, as calculated using Simpson's diversity index. The ARG-MOB ratio is calculated as the mean of the four mobilization parameters (MOB) and ranges from 0 to 1 with 1 representing very high mobilization, signified by very high IS and plasmid ratios, frequent association with integrons, and a wide phylogenetic dispersal across genera. Fig. 4 shows the MOB parameters and ARG-MOB scale. For each parameter, boxplots with MWU test results are also shown.

The median ARG-MOB per major mechanism is highest for ATR $(p<0.0001)$, while AE has a low median ARG-MOB but not significantly different from ATA and Al groups. Antibiotic target protection (ATP) and Al groups are not significantly different (Fig. 4a).

\section{Efflux genes are rarely mobilized}

The AE mechanism has the lowest median ARG-MOB (although only significantly lower than ATR and ATP), which is reflected by median Replicon, IS, and Integron ratios that are lower than most other groups (Fig. 4), i.e. efflux genes are rarely mobilized by these MGEs. It is therefore likely that most identified AE ARGs are housekeeping efflux pumps located in conserved loci of chromosomes, with a few exceptions (e.g. multidrug efflux genes oqxAB ${ }^{34-38}$ ). This warrants caution to include considerations of genetic context when screening environments for efflux-associated ARGs.

The highest median ARG-MOB mechanism, ATR, is characterized by AROs with a high degree of mobilization by IS elements and plasmids (Fig. 4). The high ARG-MOB ATR AROs are furthermore strongly associated with integrons and are taxonomically more wide-spread than ATP, AI, and AE groups. Likewise, some ATP AROs are highly mobilized and widespread but they are to a lesser degree associated with integrons. Generally, ATR is significantly more associated with integrons than other categories, although the median of Al is higher than ATA and AE.

AROs of Al mechanism are the least phylogenetically dispersed but are instead conserved within few genera, as indicated by the lowest median Simpson index. Possibly, many genes and/or proteins under the Al mechanism only function in specific genera, whereas those of other mechanisms can function in wider ranges of genera. While there certainly are AI AROs that have been mobilized by 
plasmids, transposons, and integrons, there are many others that have not been decontextualized

213 (Fig. 4). All major mechanisms have exceptions in the form of AROs with elevated ARG-MOB, as evaluated on all four parameters, although ATA, ATP, and AE have few or no AROs with ARG-MOB higher than 0.75 .

\section{The ARG-MOB scale proficiently describes decontextualization of putative ARGs}

218 The four MOB parameters all correlate significantly with each other, showing that they covary and are appropriate for calculating the ARG-MOB scale (Fig. 5a,b). Hierarchical clustering of AROs from all five major mechanisms in a heatmap shows apparent mechanism-specific profiles of ARG-MOB scores, as well as each of the four MOB parameters (Fig. 5c).Two major branches are formed from clustering: I) a high-ARG-MOB branch dominated by Al, as well as other individual AROs from other mechanisms and II) a low-MOB branch mostly populated by AE AROs.

The highest correlation coefficient is seen for IS-Replicon ratios, showing that ARGs placed on plasmids are likely mobilized by IS elements prior to insertion on plasmids (Fig. 5a,b). The second highest correlation is found between IS and integron ratios, indicating that ARGs, found as gene cassettes in integrons, are likely to have been mobilized (as part of integrons) by IS elements (Fig. $5 a, b)$ which has been often reported and discussed ${ }^{4,24,32}$. To a lesser degree, ARGs found on plasmids are correlated with integrons.

Perhaps not surprisingly, the Simpson diversity index correlates positively with IS, replicon, and integron ratios (Fig. 5a). This shows directly that highly mobilized ARGs and those found in integrons are also likely to be phylogenetically widespread. On the basis of these correlations, we conclude that the ARG-MOB ratio proficiently describes decontextualization of putative ARGs. Pearson correlation coefficients and MGE co-occurrences were also calculated per mechanism (Supplementary Note 9).

\section{Some AROs are highly divergent in mobilization}

Many AROs can be defined as either highly mobilized or only to a very little degree. Still, some AROs have a very large spread from their ARG-MOB score, showing that they are most often sitting unmobilized on a chromosome, but have one or times been mobilized and widely dispersed 
242 (Supplementary Figs. 22,23). This is exemplified by the efflux pump encoded by genes oqxAB (ARO3003922-3) 34,35 (Supplementary Note 10). These genes are found on essentially all Klebsiella pneumoniae chromosomes where they do not confer resistance unless highly overexpressed ${ }^{36-38}$. However, they were shown to be mobilized by IS elements on plasmid pOLA52 where they confer resistance to multiple antibiotics ${ }^{34}$. The oqx AROs show high spread across their mean IS and Replicon ratios (oqxA has ratios of 0.35 and 0.16 , respectively), showing that mean ratios are low due to Klebsiella chromosomes but that there are many outliers due to variants in Escherichia and Salmonella that are only found mobilized by IS elements and usually on plasmids (Supplementary Fig. 22; Supplementary Table 5).

Outliers from the mean of IS and Replicon ratios can also be considered per genus instead of ARO, in order to highlight that ARGs in some genera are much more mobilized than in others. For example, efflux pump genes in Shigella are much more associated with IS elements compared to the global average, but they are not found on plasmids more than on average (Supplementary Fig. 23). Likewise, many Al ARGs are found more on plasmids in Escherichia, Salmonella, Klebsiella, Citrobacter, and Enterobacter than their respective average placements per ARO. Other genera including Proteus, Pseudomonas, Acinetobacter, and Morganella tend to have some Al ARGs more located on chromosomes than the given ARO average, indicating that chromosomes in these genera may be considered reservoirs of genes with potential as resistance determinants. This highlights the complexity of the ARG issue and emphasises the importance of considering genetic context of ARGs before predicting resistance.

\section{Defining ARG-MOB categories}

265 Smoothed kernel density estimates of AROs and their ARG-MOB values are shown in Fig. 6a per mechanism and cumulatively for all mechanisms. The following five ARG-MOB groupings were defined computationally: Very low (ARG-MOB =0), Low $(0<\mathrm{ARG-MOB}<0.182)$, Medium $(0.182<$ ARG-MOB < 0.378), High (0.378 < ARG-MOB < 0.681), Very high (ARG-MOB > 0.681). These definitions are largely the same when estimating per mechanism individually (Supplementary Fig. 24). Numerically, AI has the highest number of High and Very high ARG-MOB AROs (144 and 54, 
respectively), while ATR has the highest percentage of High and Very high ARG-MOB AROs with these categories representing $64 \%$ of ATR AROs (Fig. 6b).

\section{High ARG-MOB AROs correspond with high-risk ARGs}

High and Very high ARG-MOB AROs (Fig. 6c) are mainly ARGs that were initially identified in very serious and resistant pathogens where they indeed confer resistance. Vice versa, many low ARGMOB AROs have only been shown to confer resistance when placed on high-expression cloning vectors but not in any natural wild-type isolate. A few examples are described below and in Supplementary Note 11. A comprehensive table for all 1,176 AROs can be found as an interactive table in Supplementary Table 6.

For all major mechanisms, many AROs are classified as Very low or Low ARG-MOB (Fig. 6c) and ATA does not have any Very high ARG-MOB AROs, while ATP has two (AR03002803 and 3002801; quinolone resistance genes qnrVC6 and qnrVC4). Al has many AROs with High and Very high ARGMOB, which include infamous $\beta$-lactamases, aminoglycoside nucleotidyltransferases (ANT), and others (Fig. 6c). With a median ARG-MOB of 0.73 , the Verone integron-encoded metallo- $\beta$ lactamase (VIM) is the $\beta$-lactamase with the highest median ARG-MOB. There are three VIM $\beta$ lactamase AROs, of which ARO3002271 has the highest ARG-MOB of any AI ARO at 0.91. In RefSeq complete genomes, it is only found inserted in integrons, and is located close to IS elements and on plasmids in $95 \%$ of the CRLs found $(n=21)$. It is dispersed across 6 unique genera for a Simpson index of 0.75 (Pseudomonas, Salmonella, Escherichia, Klebsiella, Citrobacter, and Enterobacter). VIM-1 was isolated from a multiresistant $E$. coli from a patient. It was inserted in a Class 1 integron and found on a conjugative plasmid ${ }^{39}$. It has since been seen in multiple Enterobacteriaceae, typically in association with MGEs, and is globally spread ${ }^{40}$. The highest ARG-MOB ATR AROs belong to the trimethoprim resistant dihydrofolate reductase $d f r$ submechanism. The ARO3003013 within this submechanism has the highest ARG-MOB of any ARO at 0.95. A Class 1 integron with dfrA15 is widespread in Vibrio cholera isolates in Africa and was found on a conjugative plasmid ${ }^{41}$. It is the ARO with the highest ARG-MOB, since it was only found to be associated with IS elements, integrons, and plasmids (all ratios $=1$ ). It has a Simpson index of 2990.82 and the 7 CRLs are dispersed across 6 genera (Vibrio, Salmonella, Enterobacter, Leclercia, Klebsiella and Escherichia). 
Based on examples of high and low ARG-MOB AROs (Supplementary Note 11), a pattern emerges that high ARG-MOB AROs were originally isolated from and identified in already virulent, pathogenic bacteria that had indeed been tested as resistant, often by acquisition of new genetic material. On the other hand, low ARG-MOB AROs were generally identified in susceptible bacteria and/or only shown to cause resistance when cloned into vectors with strong gene expression. This warrants caution when choosing ARGs of interest in either targeted (q)PCR screening or metagenomic sequencing of environmental samples.

\section{Discussion}

Ideally, the genetic context is always included in ARG predictions, since even the highest ARGMOB scoring genes will have representatives that are not decontextualized and may not confer resistance. This study documents how genes from even the most mobilized categories of ARGs can be found unmobilized on chromosomes. Our results clearly demonstrate that the validity of using PCR-based screening to assess the abundance and distribution of putative ARGs is questionable at best (e.g. for antibiotic inactivation ARGs) and almost certainly miscalling others (antibiotic efflux ARGs). This is in agreement with previous studies finding discrepancies between genotypic and phenotypic resistance predictions ${ }^{8-12}$, with especially efflux-related markers producing a high number of false-positive predictions ${ }^{14}$. Therefore, it is necessary to consider the genetic context of putative ARGs when trying to assess the relevance of $A R G s^{1,3,26}$. This could be achieved by applying PCR primers that target regions spanning both an $A R G$ and an associated MGE ${ }^{42}$. For more accurate ARG calling, metagenomic sequencing using long-read platforms is a prerequisite to enable the detection of ARGs and their genetic contexts.

\section{Methods}

\section{Databases and ARG prediction}

All code for data processing was written in BASH scripts and statistics and plotting were primarily done in RStudio. All scripts and a searchable table of results (Supplementary Table 6) are available at https://github.com/tueknielsen/ARG-MOB. The details of the methods are described below. 
All complete bacterial genomes (15,790 entries with 16,785 chromosomes and 14,280 plasmids) were downloaded from RefSeq on Dec. 122019 using the ncbi-genome-download tool v0.2.11 (available from https://github.com/kblin/ncbi-genome-download). In order to ensure uniform prediction of genes across all bacterial genomes, Prodigal ${ }^{43}(\mathrm{v} 2.6 .3)$ was used to predict genes from nucleotide sequences and write corresponding amino acid sequences from all RefSeq genomes. Since Prodigal first trains itself based on the input sequence, gene prediction was performed on subsets of each genus present in RefSeq genomes. Per genus, two rounds of Prodigal were performed with the -meta flag enabled in the second run, as it predicts some genes that are missed in single genome mode and vice versa. Results from the "single" and "meta" gene predictions were combined and redundant annotations found with both methods were merged.

Several ARG databases and tools for predicting ARGs have been produced, including CARD ${ }^{44}$, ARDB $^{45}$, MEGARes $^{46}$, ResFinder ${ }^{47}$, SARG $^{48}$, ARG-ANNOT $^{49}$, DeepARG-DB $^{50}$, ARG-miner $^{51}$, FARME $^{52}$, and others. Some are discontinued while others receive updates occasionally. The CARD database is large, actively updated, well-curated, and widely used. Furthermore, it makes use of ontology terms (Antibiotic Resistance Ontology: ARO) that allow for the grouping of resistance genes according to resistance mechanisms. Because of these advantages over other databases and the essential role of ontology terms, the CARD database was used in this study. The "protein homolog" models from CARD were used here, since they do not contain resistance determinants that are based on mutations. The main resistance mechanisms defined in the CARD database are "antibiotic efflux" (AE), "antibiotic inactivation" (Al), "antibiotic target alteration" (ATA), "antibiotic target protection" (ATP), "antibiotic target replacement" (ATR), and the less abundant "reduced permeability to antibiotic" (RPA). A few additional categories exist that are hybrids of two of the above mechanisms, but there are very few entries of these in CARD and are for most of the analyses not considered.

357 The Comprehensive Antibiotic Resistance Database (CARD v3.0.7) was downloaded and only the protein homolog model was used in this study, excluding resistance determinants related to sequence variants (e.g. SNPs). DIAMOND ${ }^{53}$ blastp was used to identify putative ARGs in all RefSeq genomes. For blastp against the CARD database, both query and subject coverages were set to a 
minimum of $80 \%$, while E-value cutoffs were set to $1 \mathrm{e}-10$, to limit the rate of spurious hits. For each query protein from all RefSeq genomes, only the single best CARD match was kept.

The CARD auxiliary tool, RGI ${ }^{54}$, for predicting ARGs in (meta)genomes uses curated blastp bitscore cutoffs unique to every ARG protein in the CARD database. The same bitscore cutoffs were applied here, with the exception that hits with bitscores lower than the RGI cutoff were included if they had an identity score and a query coverage of at least $80 \%$. These hits were included in order to keep more putative ARG hits from environmental bacteria that are not clinically relevant, since it is assumed that CARD and other ARG databases are biased towards genes that reside in anthropogenically relevant strains. Blastp hits with bitscores above the RGI cutoff were also only kept if query coverage was at least $80 \%$. The effects of these filters are further described in Supplementary Information (Supplementary Figs. 1,2,3; Supplementary Note 1).

Data tables were imported into $\mathrm{R}$ for statistics and visualization using the packages ggplot $2^{55}$, dplyr ${ }^{56}$, tidyr $^{57}$, gridExtra $^{58}$, ggpubr $^{59}$, ggExtra $^{60}$, reshape $^{61}$, knitr $^{62}$, kableExtra $^{63}$, vegan $^{64}$, PerformanceAnalytics ${ }^{65}$, ComplexHeatmap ${ }^{66}$, RColorBrewer $^{67}, \mathrm{DT}^{68}$, rstatix $^{69}$, tidyverse $^{70}$, broom ${ }^{71}$, and plotly ${ }^{72}$. All statistical tests on rank-sums of groupings were performed with unpaired MannWhitney U-tests (MWU) with Benjamin-Hochberg FDR correction for multiple testing.

\section{Extracting the genetic context of ARGs}

The average length of composite and unit transposons were calculated based on 449 entries in the Transposon Registry ${ }^{73}$. This average $(12.17 \mathrm{kbp})$ was used as the maximum allowed distance between an ARG and an IS element for classifying an association (Supplementary Table 1). However, since ARGs in transposons can be on either strand relative to the transposase, IS elements are identified within $12.17 \mathrm{kbp}$ of an ARG in both directions. This enables searching for transposons of up to $24.34 \mathrm{kbp}$ (plus the length of the identified ARG), which would include $77.73 \%$ of the 449 composite and unit transposons in The Transposon Registry ${ }^{73}$ (Supplementary Fig. 4).

For all filtered blastp ARO hits, up to 12,170 bp both up- and downstream of the hit were extracted from the respective RefSeq replicon using the faidx command from Samtools ${ }^{74}$ (v1.9-166-g74718c2). 
If an ARG was found within $12.17 \mathrm{kbp}$ of either terminus of a replicon, only sequence until the terminus was extracted and not continued from the other end of the sequence, since entries in RefSeq complete genomes may not be actually complete, due to low sequencing coverage regions stemming from e.g. GC-bias in sequencing ${ }^{75}$. Loci were categorized according to the ARO of the identified ARG. There are 9 ARO major mechanism categories of which three are less abundant "hybrids" merged by two other categories. The 6 non-hybrid categories Antibiotic efflux (AE), Antibiotic inactivation (Al), Antibiotic target alteration (ATA), Antibiotic target replacement (ATR), Antibiotic target protection (ATP), and Reduced permeability to antibiotic (RPA) are here considered the main categories and are the ones mainly investigated in this study. The mechanism "Reduced permeability to antibiotic" is only represented by three ARO categories and is excluded from some statistical analyses.

\section{IS elements in ARG loci and 16S rRNA as control}

IS elements in ARG loci were predicted using DIAMOND blastp against the ISfinder database ${ }^{76}$, as implemented in Prokka ${ }^{77}$ (v1.14.0). The same E-value cutoff for IS annotations, as Prokka applies during gene annotation (1e-30), was used here and the minimum query coverage accepted was 90\%. Only the top IS hit for each query protein was kept, since multiple "good" hits to distinct IS families may occur per query. The distance between a given putative ARG and its closest IS neighbour within $12.17 \mathrm{kbp}$ in either direction (if any) was calculated without considering the coding strand of the genes. ARGs not within $12.17 \mathrm{kbp}$ of an IS element were not considered when calculating the mean ARG-IS element distances.

Since $16 \mathrm{~S}$ rRNA genes are not expected to be often mobilized by IS elements, the distance between $16 S$ rRNA genes and IS elements were explored in all complete RefSeq bacterial genomes, in order to assess how many "false-positive" ARG-IS associations are expected to be identified using the predicted using barrnap ${ }^{78}$. Of these, $94.61 \%$ did not have identified IS elements within 12,170 bp in either direction, which can be seen as analogous to a $95 \%$ confidence interval for predicting 


\section{Clustering ARG loci to remove redundancy}

422 Extracted loci with putative ARGs were grouped based on the CARD ARO category of the loci ARGs. 423 In order to remove redundancy from database bias (Supplementary Notes 3 and 4; Supplementary 424 Figs. 6,7) towards oversampling of e.g. almost identical E. coli chromosomes, extracted loci were 425 clustered with USEARCH ${ }^{79}$ (v11.0.667_i86linux64) (Supplementary Fig. 8 and Supplementary Table 426 2). Per ARO group, sequence loci were clustered into what we refer to here as Clustered Resistance 427 Locus (CRL) using the "-cluster_fast" command with the criteria that sequences in a cluster are at 428 least 99\% similar over at least $90 \%$ of the length (both target- and query coverage) and only the 429 single best hit was allowed per sequence. The "-sort length" flag was also enabled to sort loci by 430 length before clustering, since loci vary in length (sum of $12.17 \mathrm{kbp}$ up- and downstream plus an 431 ARG of varying length). This ensures that loci of identical length (with the exact same ARG) are 432 merged into the same CRLs. For each CRL, the centroid sequence was used as representative 433 sequence for downstream analyses.

\section{Integron prediction}

Integrons and cassette arrays were predicted using IntegronFinder ${ }^{31}$ using the centroid CRL sequences as input. IntegronFinder can predict complete integrons including gene cassettes, In0 elements where only integrase is present, and CALINs (Cluster of attC sites Lacking Integrase nearby). All three classes of integrons are included in the analyses and no distinction is made, since a putative ARG observed in e.g. a CALIN has been previously associated with an integron and may still be in related strains.

\section{MOB metrics and the ARG-MOB scale}

444 Four main metrics (Fig. 2), or ratios (0 to 1), of mobilization were calculated per ARO that aim to quantify just how mobilized groups of ARGs are. These four ratios are A) the Replicon ratio B) the IS ratio C) the Integron ratio and D) the phylogenetic spread of an ARO across distinct genera, 447 quantified by the Simpson diversity index. Pearson correlation coefficients between MOB metrics were calculated. 
For each ARO category, the number of CRLs with and without identified IS elements were counted and the IS ratio was derived where an IS ratio of 1 indicates that all CRLs belonging to a given ARO have an IS element within 12,170 bp either up- or downstream of the ARG. Vice versa, an IS ratio of 0 indicates that none of the CRLs in an ARO have IS elements in proximity. Similarly, the Replicon ratio was calculated per ARO based on the CRLs' location on either plasmids or chromosomes. A Replicon ratio of 1 means that all CRLs in a given ARO are of plasmid origin and a 0 means that all CRLs are from chromosomes. The Integron ratio indicates how many CRLs are inserted in integrons per ARO. For measuring the taxonomic distribution of each ARO category, the Simpson diversity index (range 0 to 1 ) was calculated per ARO using unclustered sequences and the genera they were identified in.

The ARG-MOB scale (0-1) represents the mean of the four MOB metrics described above and serves as a ranking scheme to evaluate how the degree to which members of an AROs have been mobilized. Based on the smoothed kernel density estimates of all ARG-MOB scores, groupings were made to categorize AROs by their ARG-MOB score. An ARG-MOB score of 0 indicates that ARGs of the given ARO were not once found to be mobilized in the RefSeq genomes and a score of 0 is thus categorized as Very low. Valleys in the density distribution of ARG-MOB scores were used to computationally pinpoint thresholds between ARG-MOB categories. The Low group ranges ARG-MOB score from 0.0 to 0.182 , the Medium group ranges from 0.182 to 0.378 , High ranges from 0.378 to 0.685 , and Very high ranges from 0.685 to 1.0 . For the Low-Medium and Medium-High cutoffs, the low point in valleys was used to define values but no apparent valley is present between High and Very high. Instead, a linear model was fitted to the right-side slope of the High peak and another fitted to the approximately linear data range starting at ARG-MOB score 0.7. The intersection between the two linear models (0.685) was used as the cutoff between the High and Very high groups (Supplementary Fig. 9). 


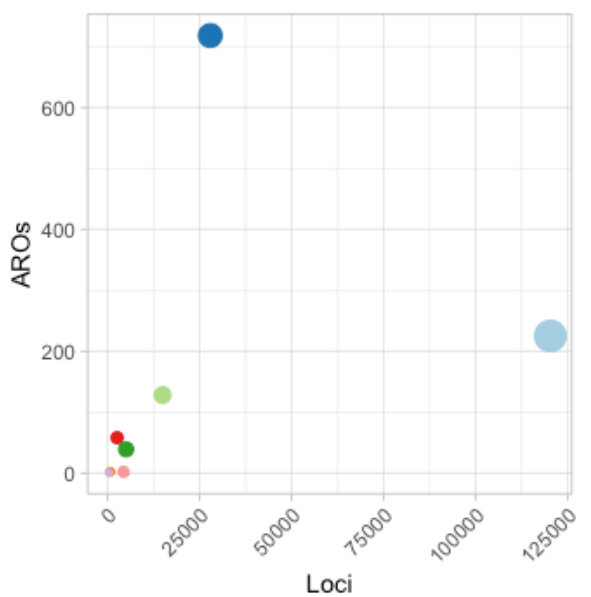

\begin{tabular}{|l|c|c|c|}
\hline \multicolumn{1}{|c|}{ Mechanism } & \multicolumn{1}{|c|}{ Loci } & \multicolumn{1}{|c|}{ CRLs } & \multicolumn{1}{|c|}{ AROs } \\
\hline Antibiotic efflux (AE) & 120336 & 28953 & 225 \\
\hline Antibiotic inactivation (AI) & 27889 & 13763 & 718 \\
\hline Antibiotic target alteration (ATA) & 14931 & 4856 & 128 \\
\hline Antibiotic target replacement (ATR) & 5009 & 3360 & 39 \\
\hline $\begin{array}{l}\text { Antibiotic efflux (AE); } \\
\text { Reduced permeability to antibiotic (RPA) }\end{array}$ & 4330 & 962 & 2 \\
\hline Antibiotic target protection (ATP) & 2559 & 1428 & 58 \\
\hline Reduced permeability to antibiotic (RPA) & 778 & 142 & 3 \\
\hline $\begin{array}{l}\text { Antibiotic target alteration (ATA); } \\
\text { Antibiotic target replacement (ATR) }\end{array}$ & 675 & 276 & 2 \\
\hline $\begin{array}{l}\text { Antibiotic target alteration (ATA); } \\
\text { Antibiotic efflux (AE) }\end{array}$ & 381 & 155 & 1 \\
\hline
\end{tabular}

479 Fig. 1: Overview of counts of ARG loci, Resistance Clustered Loci (CRLs), and CARD Antibiotic 480 Resistance Ontology terms (AROs). In the plot, points are sized according to their CRL count (see 481 table). Row colours in table correspond to their point colours in the left plot. The three hybrid mechanisms AE;RPA, ATA;ATR, and ATA;AE, as well as the low-ARO RPA mechanism, are excluded 483 from some analyses, as they are not considered "main mechanisms". 

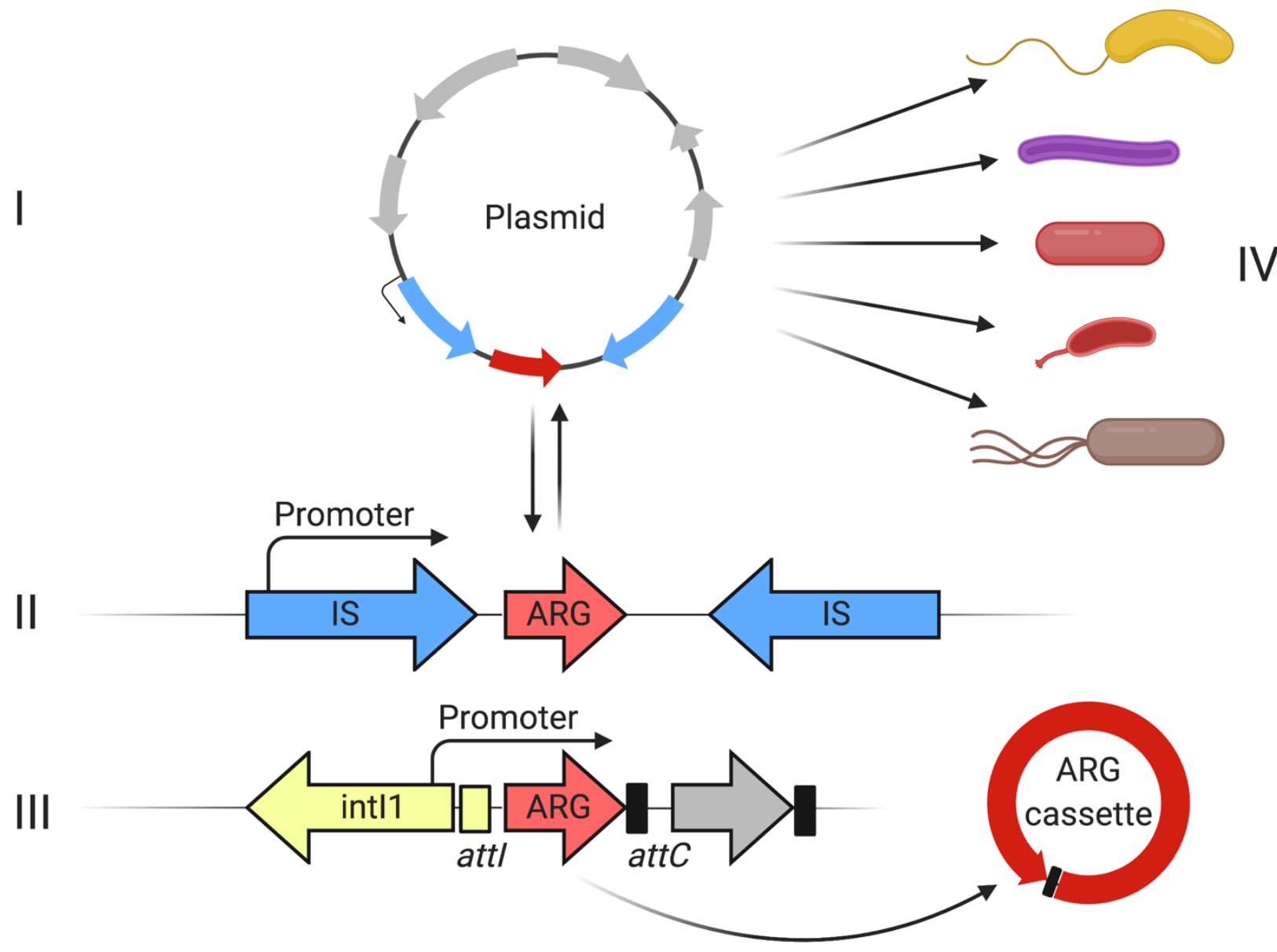

Fig. 2: The four investigated mobilization parameters. I) classification of the replicon type that an ARG loci was found on, II) presence of one or more IS elements within 12,170 bp either up- or downstream of ARG, III) association of found ARG with integrons, and IV) the phylogenetic spread across genera, calculated by the Simpson diversity index. ARGs residing on plasmids can be rapidly spread horizontally and, in the case of multicopy plasmids, may be under heterologous expression.

Many IS elements have either an internal promoter that can overexpress accessory genes or they may contain an outward-facing -35 component that can form a hybrid promoter, if the IS element is inserted close to a -10 box. If inserted as a gene cassette in an integron, the ARG is likely overexpressed by the common integron promoter. Furthermore, a gene cassette containing an ARG may form circular DNA molecules from the integron cassette array that can be shuffled to other locations. The final factor considered in this study with regards to mobilization of ARGs is the already observed phylogenetic dispersal of said ARGs across the genera represented in the RefSeq complete 


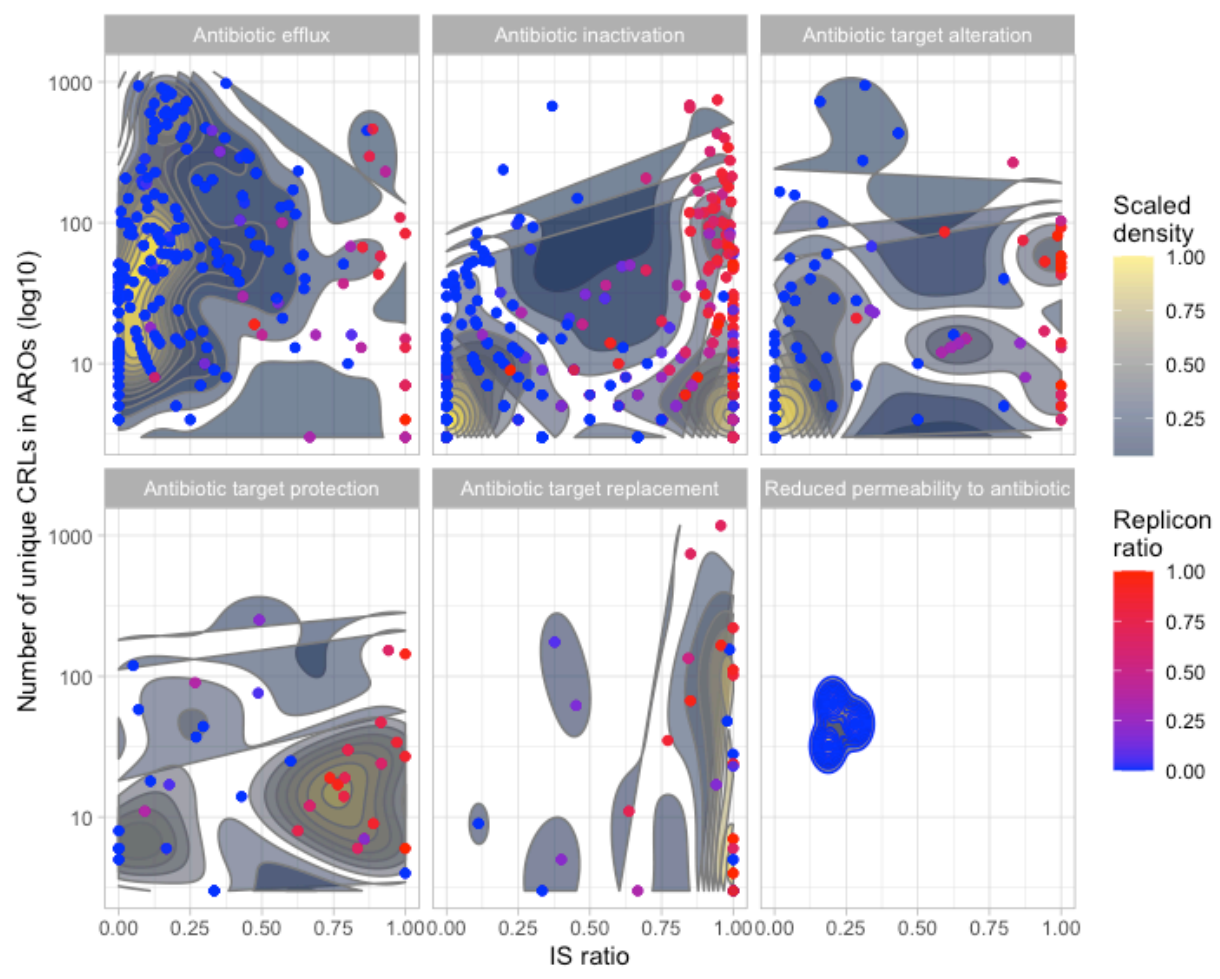

501 Fig. 3: Density plots of IS ratio against the number of unique clustered CRLs in a given ARO

502 category, represented by individual points. Plots are divided into the individual mechanisms and 503 are colored according to the Replicon ratio, where a high ratio (red) indicates that an ARO is more 504 often found on plasmids and a low ratio (blue) indicates that an ARO is more on chromosomes. 505 Density estimates are calculated with two-dimensional kernel density estimation, as implemented 506 in the stat_density_2d function under the ggplot R package. The hybrid mechanisms are not included. 

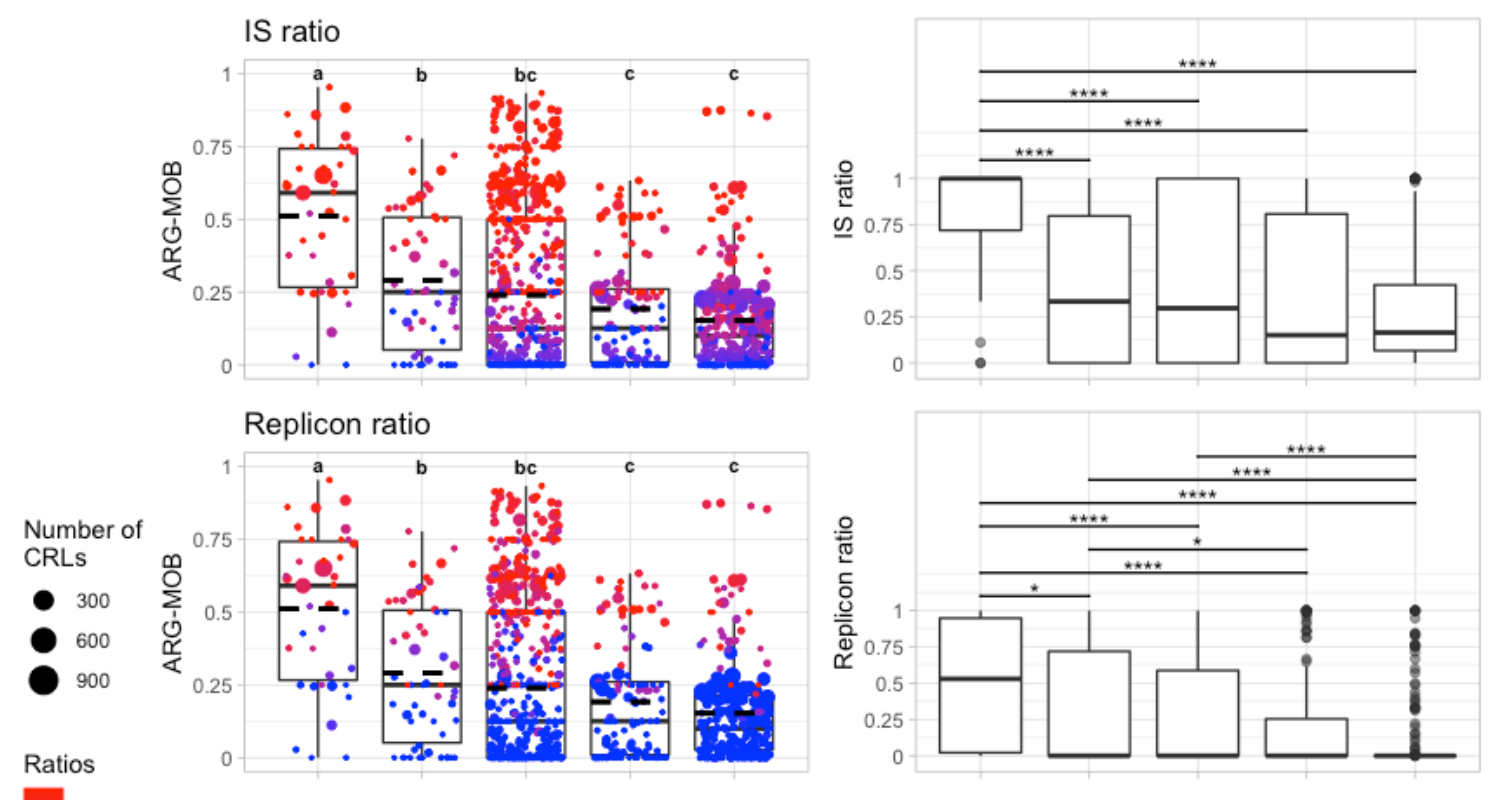

-0.8
-0.6
-0.4
-0.2
0.0
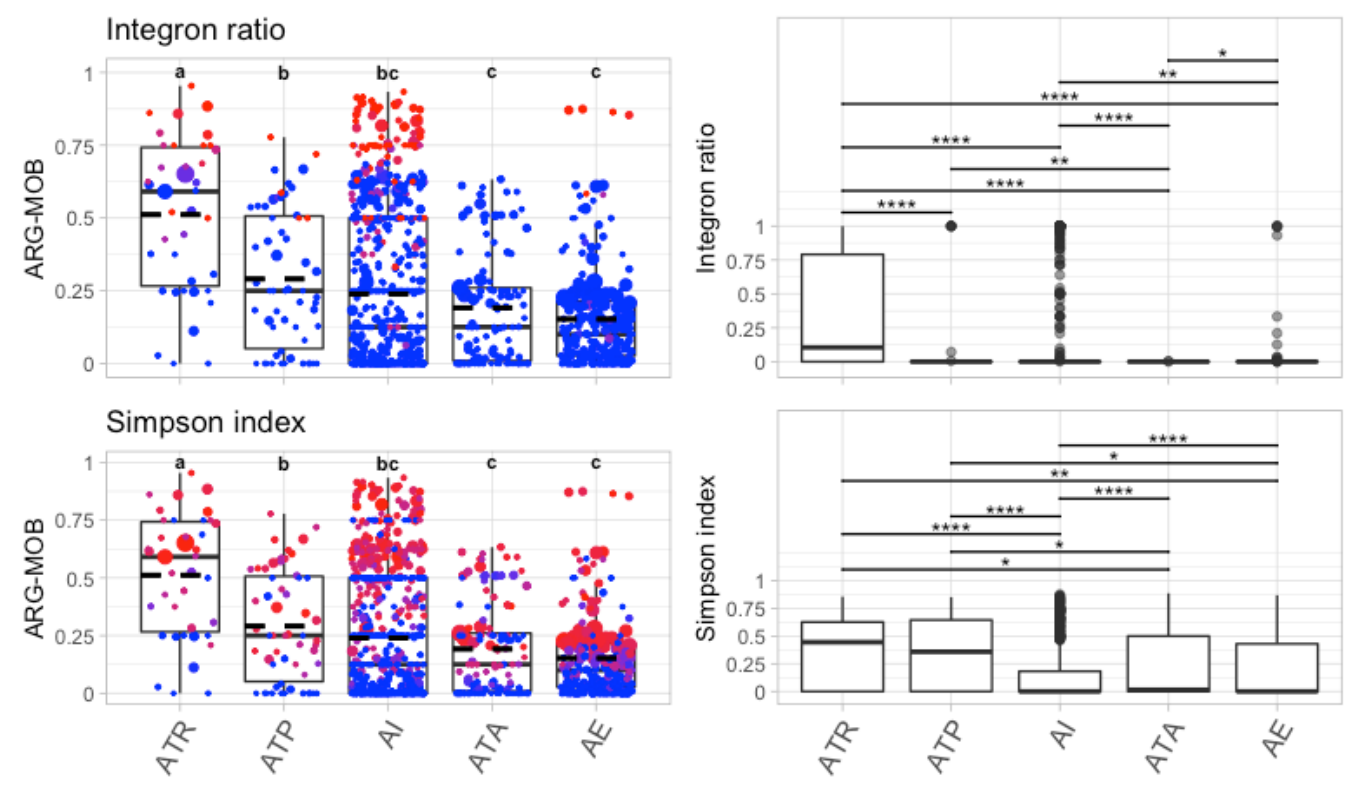

Fig. 4: ARG-MOB scores of major resistance mechanisms defined from the four MOB parameters. a, ARG-MOB score of AROs by major mechanism. Each point indicates a specific ARO and the size of 512 the point corresponds to the number of unique CRLs in that ARO. Each of the four plots shows one 513 of the individual MOB parameters as coloured gradients of the points. Points are horizontally 514 jittered but placed identically between the four plots in the left column. Mean is shown with dashed 515 lines. Boxes indicate first and third quartiles ( $25 \%$ and $75 \%$ of data) and horizontal line in box shows 516 the median. Whiskers extend to $1.5 *$ of the interquartile ranges. Letters above boxplots indicate 
significant differences between mechanism populations (Mann-Whitney U-test with FDR correction;

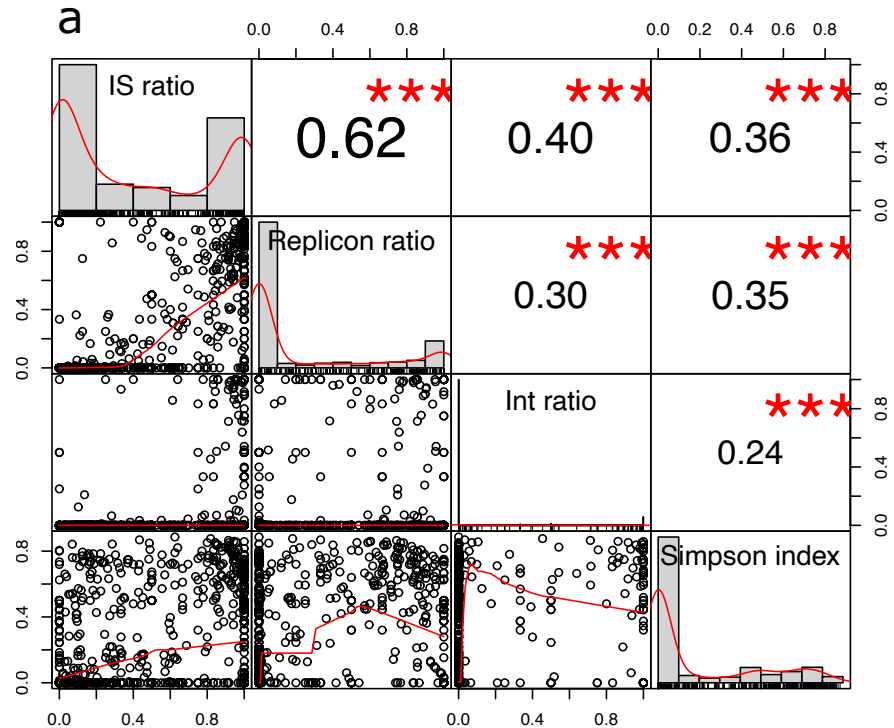

C

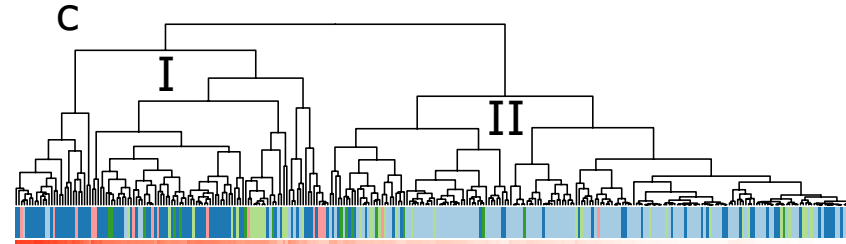

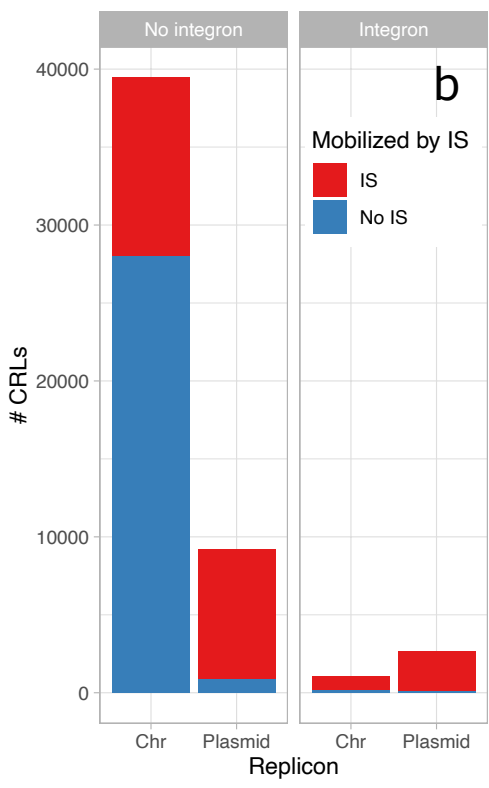

Mechanism ARG-MOB

IS ratio

Antibiotic target replacement

Antibiotic target alteratio

Antibiotic inactivation

Replicon ratio

ARG-MOB Ratio

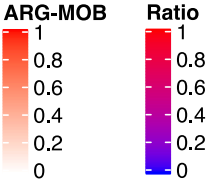

523 Fig. 5: Correlation and co-occurrence of MOB parameters. a, Pearson correlation coefficients between MOB parameters. Scatter plots between pairwise MOB parameters are shown in lower left corner. The diagonal shows histograms of distribution of each MOB parameter. The values in the upper right corner show the Pearson correlation coefficients with significance levels ( $\left.{ }^{* * *}: \mathrm{P}<0.001\right)$. b, Barplot of mobilization of unique CRLs by IS elements, plasmids, and integrons. c, Heatmap of highly abundant AROs with at least 20 CRLs. The dendrogram shows clustering of the AROs, based 
529 on the four MOB parameters, and was calculated using standard parameters in the 530 'ComplexHeatmap' package (complete hierarchical clustering on Euclidean distances).
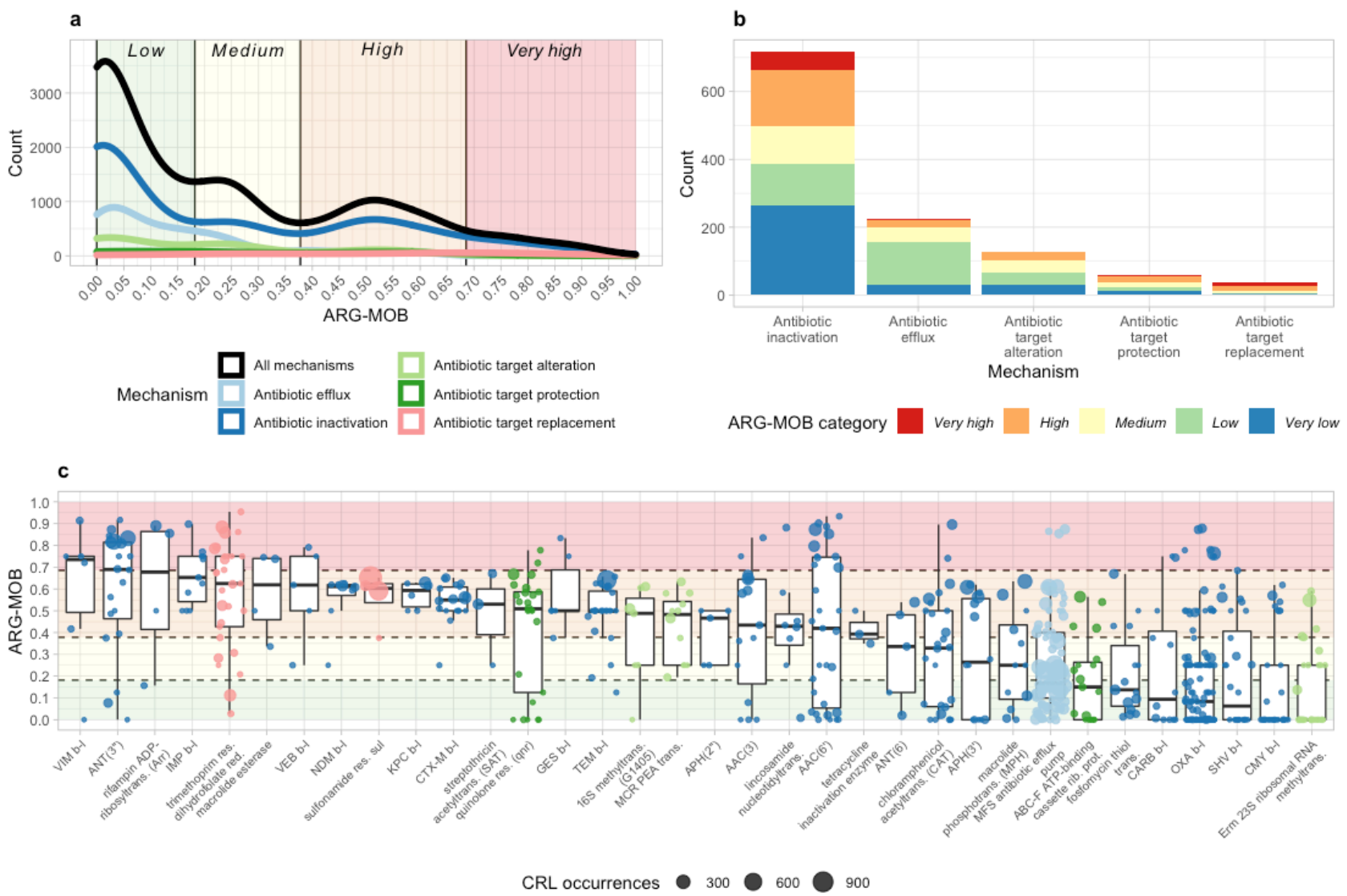

Fig. 6: Density of ARG-MOB categories and distribution per major resistance mechanism. a, Count density of ARG-MOB per mechanism. Only major non-hybrid mechanisms are shown. ARG-MOB categories were defined computationally by finding valleys in "All mechanisms" density estimates, except for the border between High and Very high where no valley is present. These calculations are described in more detail in Methods and Supplementary Fig. 9. b, Count of each ARG-MOB category per mechanism. c, All submechanisms that have AROs with High or Very high ARG-MOB scores. Points are coloured by major resistance mechanism as in Fig. $6 a$ and sized according to the number of CRLs of the given ARO. The background is coloured similarly to fig. 6a, representing Low, Medium, High, and Very high categories. The Very low category is for ARG-MOB $=0$ and does thus not have a colour in the graph. 


\section{References}

1. Martínez, J. L., Coque, T. M. \& Baquero, F. What is a resistance gene? Ranking risk in resistomes. Nature Reviews Microbiology (2015). doi:10.1038/nrmicro3399

2. Blanco, P. et al. Bacterial Multidrug Efflux Pumps: Much More Than Antibiotic Resistance Determinants. Microorganisms (2016). doi:10.3390/microorganisms4010014

3. Dantas, G. \& Sommer, M. O. A. Context matters - the complex interplay between resistome genotypes and resistance phenotypes. Current Opinion in Microbiology (2012). doi:10.1016/j.mib.2012.07.004

4. Partridge, S. R., Kwong, S. M., Firth, N. \& Jensen, S. O. Mobile genetic elements associated with antimicrobial resistance. Clinical Microbiology Reviews (2018). doi:10.1128/CMR.00088-17

5. Waglechner, N. \& Wright, G. D. Antibiotic resistance: It's bad, but why isn't it worse? BMC Biology (2017). doi:10.1186/s12915-017-0423-1

6. Dcosta, V. M. et al. Antibiotic resistance is ancient. Nature (2011). doi:10.1038/nature10388

7. Allen, H. K., Moe, L. A., Rodbumrer, J., Gaarder, A. \& Handelsman, J. Functional metagenomics reveals diverse B-lactamases in a remote Alaskan soil. ISME J. (2009). doi:10.1038/ismej.2008.86

8. Clemente, J. C. et al. The microbiome of uncontacted Amerindians. Sci. Adv. (2015). doi:10.1126/sciadv.1500183

9. Bengtsson-Palme, J. et al. The human gut microbiome as a transporter of antibiotic resistance genes between continents. Antimicrob. Agents Chemother. (2015). doi:10.1128/AAC.00933-15

10. Hughes, D. \& Andersson, D. I. Environmental and genetic modulation of the phenotypic expression of antibiotic resistance. FEMS Microbiology Reviews (2017). doi:10.1093/femsre/fux004

11. Moran, R. A., Anantham, S., Holt, K. E. \& Hall, R. M. Prediction of antibiotic resistance from antibiotic resistance genes detected in antibiotic-resistant commensal Escherichia coli using PCR or WGS. J. Antimicrob. Chemother. (2017). doi:10.1093/jac/dkw511

12. Thomas, M. et al. Whole genome sequencing-based detection of antimicrobial resistance and virulence in non-typhoidal Salmonella enterica isolated from wildlife. Gut Pathog. 
(2017). doi:10.1186/s13099-017-0213-x

13. Kos, V. N. et al. The resistome of Pseudomonas seudomonas aeruginosa in relationship to phenotypic susceptibility. Antimicrob. Agents Chemother. (2015). doi:10.1128/AAC.0395414

14. Mahfouz, N., Ferreira, I., Beisken, S., von Haeseler, A. \& Posch, A. E. Large-scale assessment of antimicrobial resistance marker databases for genetic phenotype prediction: a systematic review. J. Antimicrob. Chemother. (2020). doi:10.1093/jac/dkaa257

15. Romero, D., Traxler, M. F., López, D. \& Kolter, R. Antibiotics as signal molecules. Chemical Reviews (2011). doi:10.1021/cr2000509

16. Hernando-Amado, S. et al. Multidrug efflux pumps as main players in intrinsic and acquired resistance to antimicrobials. Drug Resistance Updates (2016). doi:10.1016/j.drup.2016.06.007

17. Henderson, T. A., Young, K. D., Denome, S. A. \& Elf, P. K. AmpC and AmpH, proteins related to the class $C \beta$-lactamases, bind penicillin and contribute to the normal morphology of Escherichia coli. J. Bacteriol. (1997). doi:10.1128/jb.179.19.6112-6121.1997

18. Mickiewicz, K. M. et al. Possible role of L-form switching in recurrent urinary tract infection. Nat. Commun. (2019). doi:10.1038/s41467-019-12359-3

19. Khedher, M. Ben et al. Massive analysis of 64,628 bacterial genomes to decipher water reservoir and origin of mobile colistin resistance genes: is there another role for these enzymes? Sci. Rep. (2020). doi:10.1038/s41598-020-63167-5

20. Sommer, M. O. A., Dantas, G. \& Church, G. M. Functional characterization of the antibiotic resistance reservoir in the human microflora. Science (80-. ). (2009). doi:10.1126/science.1176950

21. Forsberg, K. J. et al. Bacterial phylogeny structures soil resistomes across habitats. Nature (2014). doi:10.1038/nature13377

22. Singleton, C. M. et al. Connecting structure to function with the recovery of over 1000 highquality activated sludge metagenome-assembled genomes encoding full-length rRNA genes using long-read sequencing. bioRxiv (2020). doi:10.1101/2020.05.12.088096

23. Gillings, M. R., Paulsen, I. T. \& Tetu, S. G. Genomics and the evolution of antibiotic resistance. Ann. N. Y. Acad. Sci. (2017). doi:10.1111/nyas.13268 
605

606

607

608

609

610

611

612

613

614

615

616

617

618

619

620

621

622

623

624

625

626

627

628

629

630

631

632

633

634

24. Siguier, P., Gourbeyre, E. \& Chandler, M. Bacterial insertion sequences: Their genomic impact and diversity. FEMS Microbiol. Rev. (2014). doi:10.1111/1574-6976.12067

25. Mahillon, J. \& Chandler, M. Insertion sequences. Microbiol. Mol. Biol. Rev. 62, 725-774 (1998).

26. Berendonk, T. U. et al. Tackling antibiotic resistance: The environmental framework. Nature Reviews Microbiology (2015). doi:10.1038/nrmicro3439

27. Sommer, M. O. A., Munck, C., Toft-Kehler, R. V. \& Andersson, D. I. Prediction of antibiotic resistance: Time for a new preclinical paradigm? Nature Reviews Microbiology (2017). doi:10.1038/nrmicro.2017.75

28. Bengtsson-Palme, J. The diversity of uncharacterized antibiotic resistance genes can be predicted from known gene variants-but not always. Microbiome (2018). doi:10.1186/s40168-018-0508-2

29. Enault, F. et al. Phages rarely encode antibiotic resistance genes: A cautionary tale for virome analyses. ISME J. (2017). doi:10.1038/ismej.2016.90

30. Botelho, J. \& Schulenburg, H. The Role of Integrative and Conjugative Elements in Antibiotic Resistance Evolution. Trends Microbiol. (2020). doi:10.1016/j.tim.2020.05.011

31. Cury, J., Jové, T., Touchon, M., Néron, B. \& Rocha, E. P. Identification and analysis of integrons and cassette arrays in bacterial genomes. Nucleic Acids Res. (2016). doi:10.1093/nar/gkw319

32. Gillings, M. R. Integrons: Past, Present, and Future. Microbiol. Mol. Biol. Rev. 78, 257 LP 277 (2014).

33. Ramirez, M. S. \& Tolmasky, M. E. Aminoglycoside modifying enzymes. Drug Resist. Updat. (2010). doi:10.1016/j.drup.2010.08.003

34. Norman, A., Hansen, L. H., She, Q. \& Sørensen, S. J. Nucleotide sequence of pOLA52: A conjugative IncX1 plasmid from Escherichia coli which enables biofilm formation and multidrug efflux. Plasmid (2008). doi:10.1016/j.plasmid.2008.03.003

35. Hansen, L. H., Johannesen, E., Burmølle, M., Sørensen, A. H. \& Sørensen, S. J. Plasmidencoded multidrug efflux pump conferring resistance to olaquindox in Escherichia coli. Antimicrob. Agents Chemother. (2004). doi:10.1128/AAC.48.9.3332-3337.2004

36. $\mathrm{Li}$, J. et al. The nature and epidemiology of OqxAB, a multidrug efflux pump. Antimicrobial 
Resistance and Infection Control (2019). doi:10.1186/s13756-019-0489-3

37. Perez, F. et al. OqxAB, a quinolone and olaquindox efflux pump, is widely distributed among multidrug-resistant Klebsiella pneumoniae isolates of human origin. Antimicrobial Agents and Chemotherapy (2013). doi:10.1128/AAC.00725-13

38. Yuan, J. et al. Prevalence of the oqxAB gene complex in Klebsiella pneumoniae and Escherichia coli clinical isolates. J. Antimicrob. Chemother. (2012). doi:10.1093/jac/dks086

39. Miriagou, V., Tzelepi, E., Gianneli, D. \& Tzouvelekis, L. S. Escherichia coli with a selftransferable, multiresistant plasmid coding for metallo- $\beta$-lactamase VIM-1. Antimicrob. Agents Chemother. (2003). doi:10.1128/AAC.47.1.395-397.2003

40. Bush, K. \& Bradford, P. A. Epidemiology of $\beta$-Lactamase-Producing Pathogens. Clin. Microbiol. Rev. 33, e00047-19 (2020).

41. Ceccarelli, D., Bani, S., Cappuccinelli, P. \& Colombo, M. M. Prevalence of aadA1 and dfrA15 class 1 integron cassettes and SXT circulation in Vibrio cholerae 01 isolates from Africa. Journal of Antimicrobial Chemotherapy (2006). doi:10.1093/jac/dkl352

42. Guillard, T. et al. Discrimination between native and Tn6010-associated oqxAB in Klebsiella spp., Raoultella spp., and other enterobacteriaceae by using a two-step strategy. Antimicrob. Agents Chemother. (2015). doi:10.1128/AAC.00669-15

43. Hyatt, D. et al. Prodigal: Prokaryotic gene recognition and translation initiation site identification. BMC Bioinformatics (2010). doi:10.1186/1471-2105-11-119

44. McArthur, A. G. et al. The comprehensive antibiotic resistance database. Antimicrob. Agents Chemother. (2013). doi:10.1128/AAC.00419-13

45. Liu, B. \& Pop, M. ARDB - Antibiotic resistance genes database. Nucleic Acids Res. (2009). doi:10.1093/nar/gkn656

46. Lakin, S. M. et al. MEGARes: An antimicrobial resistance database for high throughput sequencing. Nucleic Acids Res. (2017). doi:10.1093/nar/gkw1009

47. Zankari, E. et al. Identification of acquired antimicrobial resistance genes. J. Antimicrob. Chemother. (2012). doi:10.1093/jac/dks261

48. Yin, X. et al. ARGs-OAP v2.0 with an expanded SARG database and Hidden Markov Models for enhancement characterization and quantification of antibiotic resistance genes in environmental metagenomes. in Bioinformatics (2018). doi:10.1093/bioinformatics/bty053 
49. Gupta, S. K. et al. ARG-annot, a new bioinformatic tool to discover antibiotic resistance genes in bacterial genomes. Antimicrob. Agents Chemother. (2014). doi:10.1128/AAC.01310-13

50. Arango-Argoty, G. et al. DeepARG: A deep learning approach for predicting antibiotic resistance genes from metagenomic data. Microbiome (2018). doi:10.1186/s40168-0180401-z

51. Argoty, G. A. A. et al. ARG-miner: A web platform for crowdsourcing-based curation of antibiotic resistance genes. bioRxiv (2018). doi:10.1101/274282

52. Wallace, J. C., Port, J. A., Smith, M. N. \& Faustman, E. M. FARME DB: A functional antibiotic resistance element database. Database (2017). doi:10.1093/database/baw165

53. Buchfink, B., Xie, C. \& Huson, D. H. Fast and sensitive protein alignment using DIAMOND. Nature Methods (2014). doi:10.1038/nmeth.3176

54. Alcock, B. P. et al. CARD 2020: Antibiotic resistome surveillance with the comprehensive antibiotic resistance database. Nucleic Acids Res. (2020). doi:10.1093/nar/gkz935

55. Wickham, H. ggplot2: Elegant Graphics for Data Analysis. Springer-Verlag New York (2016).

56. Wickham, H., François, R., Henry, L. \& Müller, K. dplyr: A Grammar of Data Manipulation. R package version. Media (2019).

57. Wickham, H. \& Henry, L. tidyr: Tidy Messy Data. R Packag. version 1.0.0 (2019).

58. Auguie, B. gridExtra: functions in Grid graphics. R Package Version 2.3. CRAN Proj. (2017).

59. Kassambara, A. ggpubr: 'ggplot2' Based Publication Ready Plots. R package version 0.1.7. https://CRAN.R-project.org/package=ggpubr (2018).

60. Attali, D. \& Baker, C. ggExtra: Add Marginal Histograms to 'ggplot2', and More 'ggplot2' Enhancements. (2019).

61. Hadley Wickham. Reshaping Data with the \{reshape\} Package. J. Stat. Softw. (2007).

62. Xie, Y. knitr: A General-Purpose Package for Dynamic Report Generation in R. (2020).

63. Zhu, H. Create Awesome HTML Table with knitr::kable and kableExtra. Create Awesome HTML Table with knitr::kable and kableExtra (2018).

64. Oksanen, J. et al. vegan: Community Ecology Package. R package version 2.4-2. Community ecology package (2019).

65. Peterson, B. G. \& Carl, P. PerformanceAnalytics: Econometric Tools for Performance and 
Risk Analysis. R package version 2.0.4. (2020).

66. Gu, Z., Eils, R. \& Schlesner, M. Complex heatmaps reveal patterns and correlations in multidimensional genomic data. Bioinformatics (2016). doi:10.1093/bioinformatics/btw313

67. Neuwirth, E. RColorBrewer: ColorBrewer Palettes. R package version 1.1-2. (2014).

68. Xie, Y., Cheng, J. \& Tan, X. DT: A Wrapper of the JavaScript Library 'DataTables'. R package version 0.13. (2020).

69. Kassambara, A. rstatix: Pipe-Friendly Framework for Basic Statistical Tests. R package version 0.5.0. (2020).

70. Wickham, H. et al. Welcome to the Tidyverse. J. Open Source Softw. (2019). doi:10.21105/joss.01686

71. Robinson, D. \& Hayes, A. broom: Convert Statistical Analysis Objects into Tidy Tibbles. R package version 0.5.5. (2020).

72. Sievert, C. Interactive Web-Based Data Visualization with R, plotly, and shiny. Interactive Web-Based Data Visualization with R, plotly, and shiny (2020). doi:10.1201/9780429447273

73. Tansirichaiya, S., Rahman, M. A. \& Roberts, A. P. The Transposon Registry. Mobile DNA (2019). doi:10.1186/s13100-019-0182-3

74. Li, H. et al. The Sequence Alignment/Map format and SAMtools. Bioinformatics (2009). doi:10.1093/bioinformatics/btp352

75. Browne, P. D. et al. GC bias affects genomic and metagenomic reconstructions, underrepresenting GC-poor organisms. Gigascience (2020). doi:10.1093/gigascience/giaa008

76. Siguier, P. ISfinder: the reference centre for bacterial insertion sequences. Nucleic Acids Res. (2006). doi:10.1093/nar/gkj014

77. Seemann, T. Prokka: Rapid prokaryotic genome annotation. Bioinformatics (2014). doi:10.1093/bioinformatics/btu153

78. Seemann, T. barrnap 0.9 : rapid ribosomal RNA prediction. https://github.com/tseemann/barrnap

79. Edgar, R. C. Search and clustering orders of magnitude faster than BLAST. Bioinformatics (2010). doi:10.1093/bioinformatics/btq461 


\section{5 \\ Supplementary information}

727 Note: Supplementary information starts with information related to Methods and is followed by

728 information related to Results. Supplementary references are listed separately from main article. 
By default, all blastp hits with bitscores exceeding the per-ARG-curated RGI bitscore-cutoffs are accepted. A ratio (bitratio) is calculated by dividing bitscores with the RGI cutoffs where a bitratio of less than 1 indicates that the blastp hit has a lower bitscore than the RGI ARG cutoff. However, as can be seen in Supplementary Fig. 1, there are many blastp hits that have high percentage identity and high query coverage, although their bitscores are below 1 and would thus be discarded if only RGI cutoffs are considered. Considering the database biases described in the article and in further detail below, it is likely that these high-similarity but low-bitratio hits are actually true ARG homologs in strains that are not related to those highly abundant in the CARD database. To include these hits, another filter was introduced where hits with bitratio lower than 1 are still included if their \% identity and \% query coverage are above 80\% (Supplementary Fig. 1).

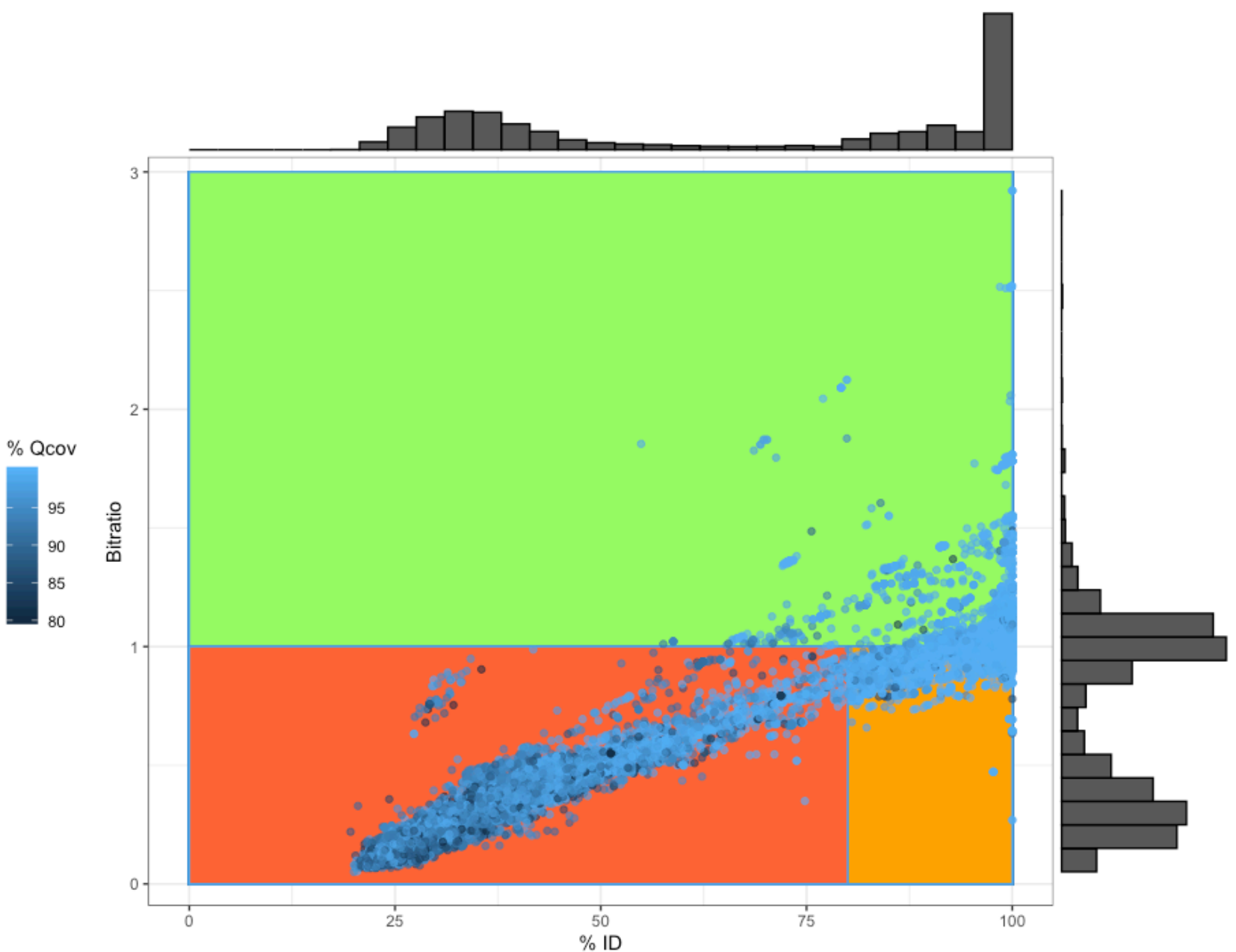


743 least $80 \%$ query coverage and an E-value of 10E-10. Subsequently, hits were filtered based on ARG-

744 specific bitscores (curated by CARD/RGI) and by minimum 80\% ID. Hits passing the RGI bitscore cutoff

745 are locateds in the green area, while hits passing the custom 80\% ID cutoff are located in the orange 746 area. Hits not passing either filter are located in the red area. Bitratio on Y-axis is calculated by 747 dividing individual bitscores by the curated bitscore-cutoffs from CARD/RGI. Histograms on the 748 outside of the plot show the distribution of both \%ID and bitratio.

750 Blastp hits passing the filters were investigated for phylogenetic distribution. By also including blastp 751 hits that are more than $80 \%$ identical to a CARD protein, we include additional 61,620 hits on top of 752 the 115,268 hits passing the RGI bitscore cutoffs. The major taxonomic orders are mostly equally 753 included by the RGI bitscore filter and the 80\% ID filter (Supplementary Fig. 2), with the biggest 754 order being Enterobacterales that constitutes $25 \%$ and $49 \%$ of blastp hits passing the ID and RGI 755 filter, respectively. 


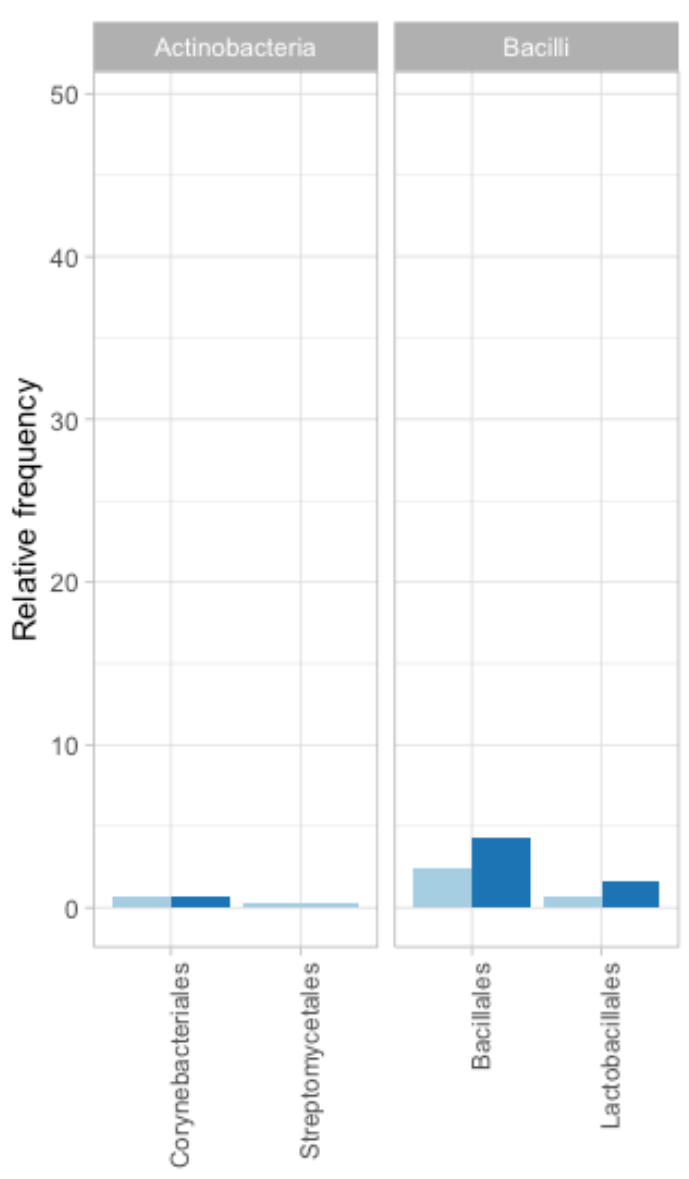
ID > 80\%).
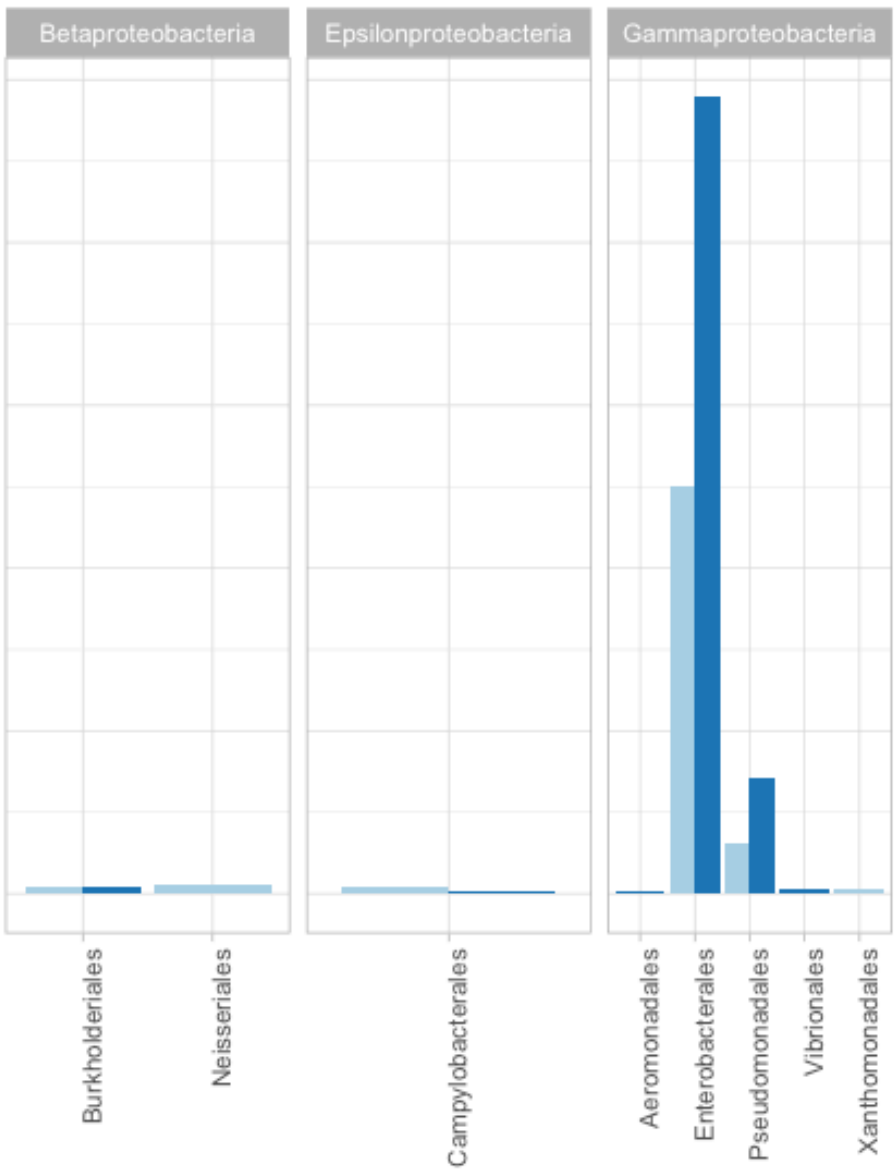

Filter

ID $>80 \%$

RGI bitscore

Supplementary Fig. 2. Major bacterial orders of ARG blastp hits passing either of the defined filters. On the $Y$-axis, the percentage of the total hits passing the respective filter is shown. Major orders are defined as orders that constitute more than $0.2 \%$ of the total data. In this plot, blastp hits can be in either ID filter or RGI filter, but not both (either bitscore $>$ threshold or bitscore $<$ threshold but

However, the minor bacterial orders are not as equally distributed in RGI and ID filters as the major orders (Supplementary Fig. 3). Specifically, the orders Micrococcales, Pseudonocardiales, Rhodospirillales, Aeromonadales, Alteromonadales, Legionellales, and Vibrionales are passing the ID filter more than the RGI bitscore cutoff. This shows that including hits passing the additional ID filter expands the scope of this study to incorporate more environmental bacteria. 

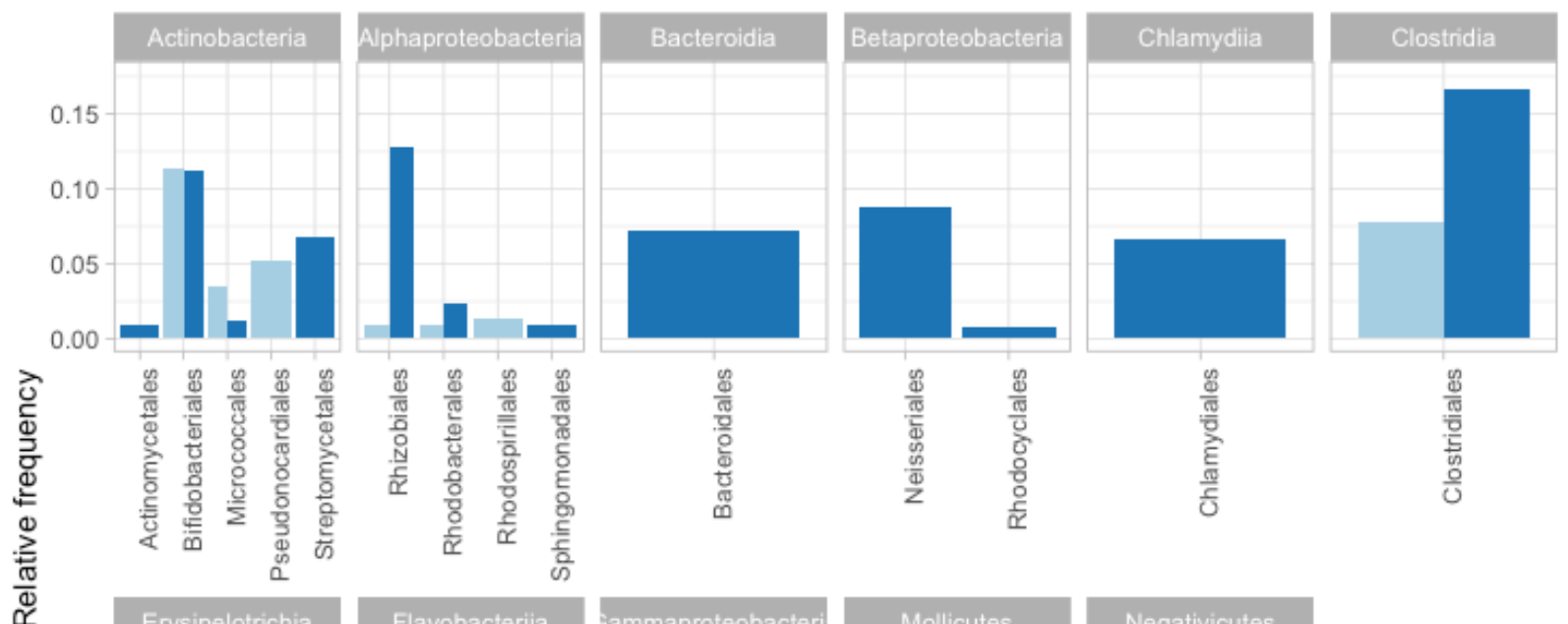

Filter

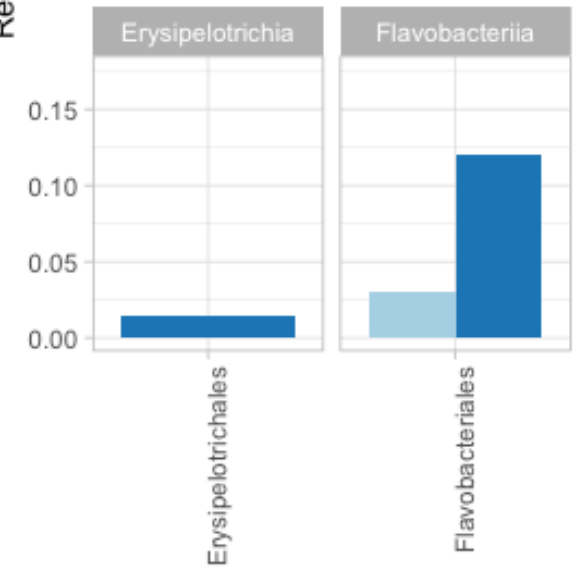

Supplementary Fig. 3. Minor bacterial orders of ARG blastp hits passing either of the defined filters.

771 On the $Y$-axis, the percentage of the total hits passing the respective filter is shown. Minor orders 772 are defined as orders that constitute less than $0.2 \%$ of the total data but only orders with more than 77310 hits are shown here. In this plot, blastp hits can be in either ID filter or RGI filter, but not both 774 (either bitscore $>$ threshold or bitscore $<$ threshold but ID $>80 \%$ ). 


\section{Mean lengths of composite and unit transposons}

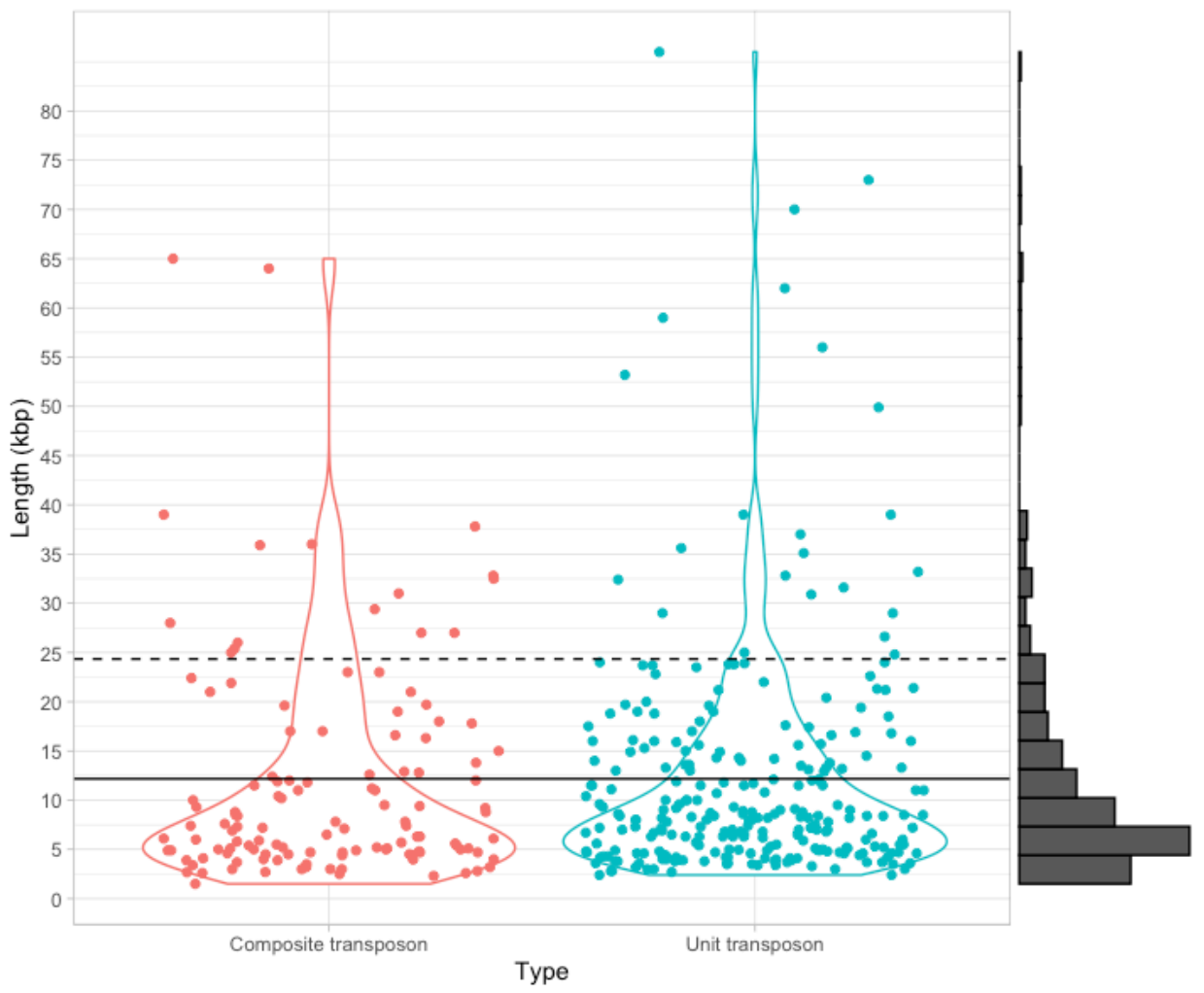

Supplementary Fig. 4. Violin plot of sequence lengths of 313 unit transposons and 136 composite transposons from The Transposon Registry1. The mean length (12.17 kbp) for both transposon types is shown as a solid line, and the maximum length of a genetic region with a putative ARG (24.34 kbp) is shown with a dashed line. A histogram of both length distributions combined is shown to the right. The individual mean lengths are 12.37 and $11.74 \mathrm{kbp}$ for unit and composite transposons, respectively.

Supplementary Table 1. Cutoffs and search criteria for ARG and IS element prediction.

\begin{tabular}{|l|l|l|}
\hline Rule & ARG hit & IS hit \\
\hline Bitscore (DIAMOND) & Higher than RGI cutoffs & NA \\
\hline E-value (DIAMOND) & $10 \mathrm{E}-10$ & $10 \mathrm{E}-30$ \\
\hline Query coverage (DIAMOND) & $80 \%$ & $90 \%$ \\
\hline Percent ID (DIAMOND) & $80 \%$ (if bitscore < RGI cutoff) & NA \\
\hline
\end{tabular}




\begin{tabular}{|l|l|}
\hline Maximum distance ARG-IS (SI) & $\begin{array}{l}\text { No higher than the mean size (12.17 kbp) of all unit and } \\
\text { composite transposons in The Transposon Registry }{ }^{1} . \text { The } \\
\text { maximum distance is investigated in both directions of ARGs for } \\
\text { IS elements and integrons. }\end{array}$ \\
\hline
\end{tabular}

\section{Supplementary Note 2: Distance between all 16S rRNA genes and closest IS elements}

Using barrnap ${ }^{2}, 80,141$ 16S rRNA genes were identified in 15,790 strains in RefSeq complete genomes (mean 5.08165 genes/genome). Only IS elements within $100 \mathrm{kbp}$ of $16 \mathrm{~S}$ rRNA genes were considered. Out of the 80,141 16S rRNA genes 4,480 had one or more IS elements within $12.17 \mathrm{kbp}$, corresponding to $5.59 \%$ (Supplementary Fig. 5). This gives an acceptable accuracy of $94.61 \%$ when predicting associations between ARGs and IS elements.

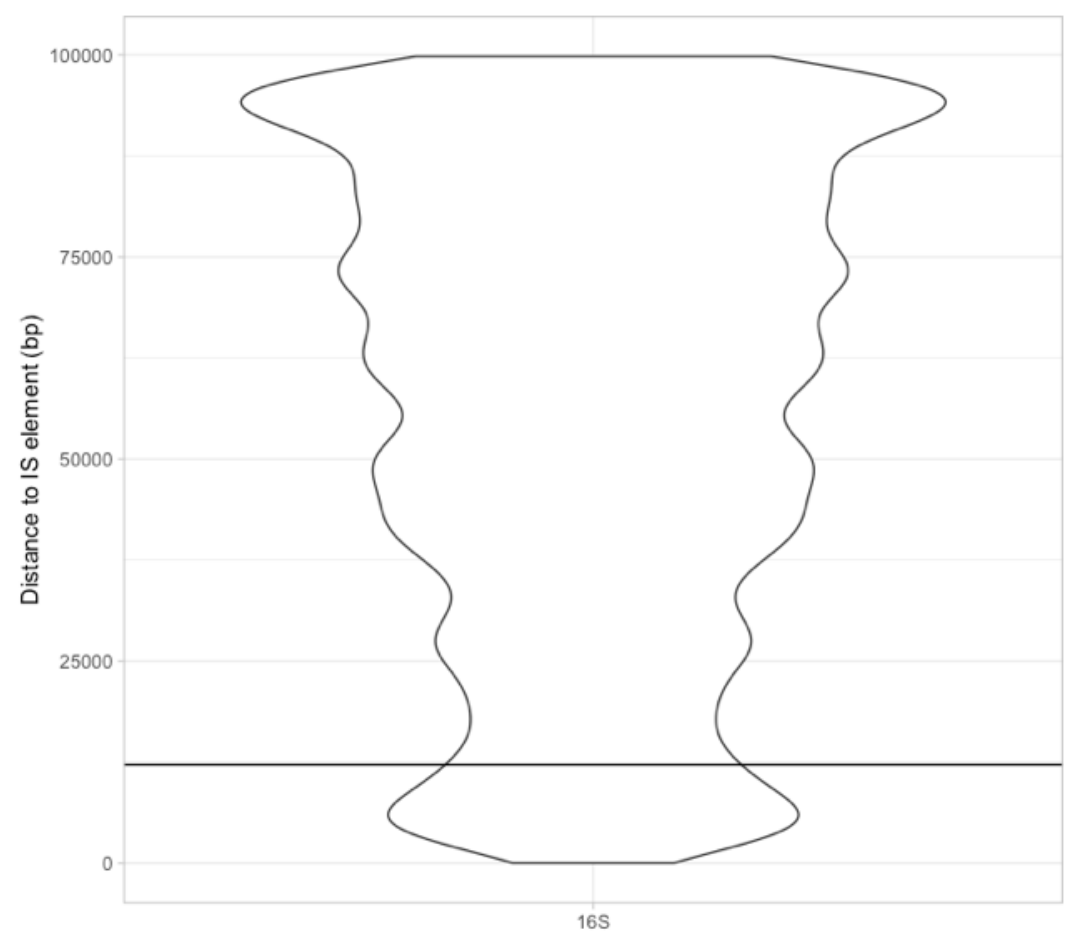

Supplementary Fig. 5. Violin plot of distances between all 16S rRNA genes and the closest IS element. 


\section{Supplementary Note 3: Database biases}

In the RefSeq complete genomes, bacteria belonging to the Enterobacterales order make up the biggest order in the database and represent 20.42\% of entries (Supplementary Fig. 6). Likewise, specialized functional gene databases, such as CARD and ISfinder, can be assumed to be biased towards certain taxonomic groups. Together, these biases will likely bias the analyses presented here synergistically by the combined biases the RefSeq, CARD, and ISfinder databases.

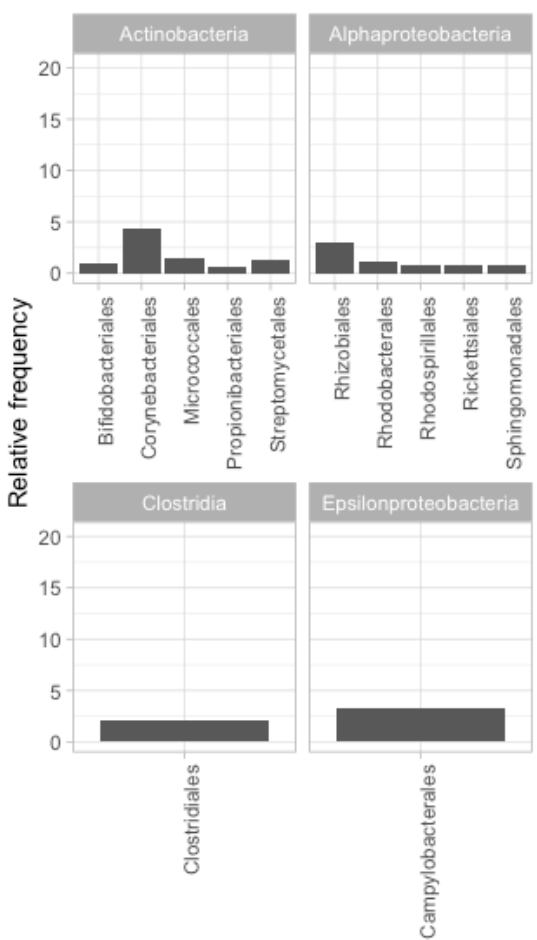

Supplementary Fig. 6. Frequencies (\%) of RefSeq genomes by order taxonomic level. Only orders making up at least $0.5 \%$ of the total abundance are shown. Orders are organized by phylum.

Proteins in the CARD database are representatives from single strains of bacteria. Comparing relative abundances of represented genera in the CARD protein homology database with the relative abundances of the same genera in the RefSeq complete genome database shows that the two databases do not have an equal distribution of genera (Supplementary Fig. 7). 


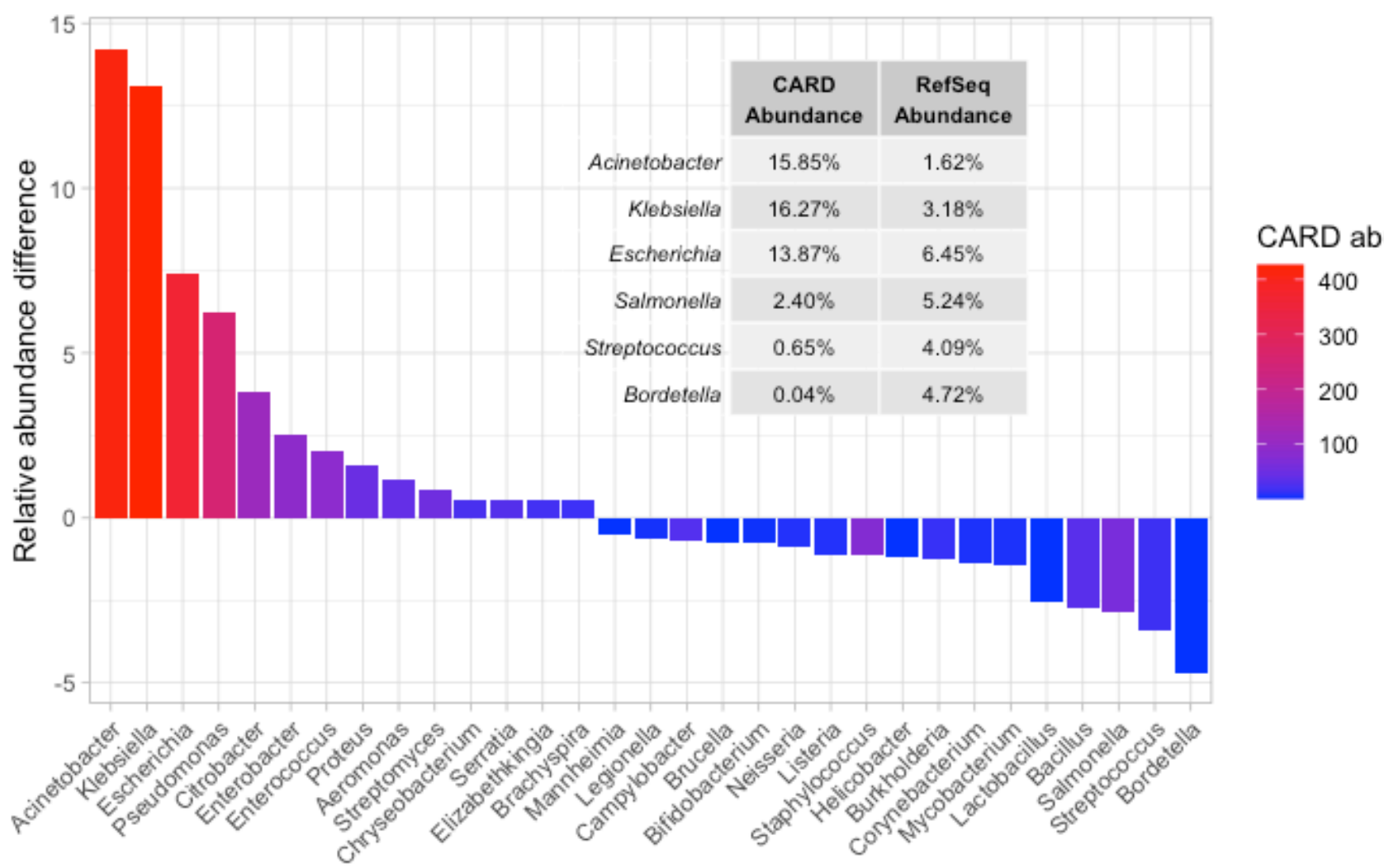

Genus

815 Supplementary Fig. 7. Differences in relative abundance in representation of genera in CARD and RefSeq complete genomes databases. Positive difference indicates higher abundance in CARD than in RefSeq and vice versa. Only genera present in both databases and with difference in relative abundance of at least $0.5 \%$ are shown.

The 6 most extreme cases with biggest difference in relative abundance between CARD and RefSeq databases are highlighted in a table within Supplementary Fig. 7. Putative ARGs from Acinetobacter strains represent $15.6 \%$ of data in CARD, while Acinetobacter only make up $1.6 \%$ of entries in RefSeq complete genomes. Conversely, Bordetella genomes constitute $4.7 \%$ of RefSeq complete genomes,

824 but are only represented by $0.04 \%$ of the CARD proteins. Streptococcus is likewise less abundant in 825 CARD. All of the top 6 most different genera are all of potential clinical relevance and there is no 826 apparent logical reason why one of these genera should have fewer or more inherent or acquired 827 ARGs than the other. Considering the absolute abundance of the CARD genera, it seems likely that 828 the differences in relative abundance between CARD and RefSeq is largely due to biased 829 representation in CARD proteins. ARG prediction in underrepresented genera such as Bordetella 
and Streptococcus is therefore likely to be less accurate and encompassing than in e.g. Acinetobater, Klebsiella, and Escherichia.

\section{Supplementary Note 4: Ameliorating database biases by clustering}

835 Most publicly available sequence databases are biased in entries towards organisms that have 836 gathered the most research interest, usually human-associated bacteria such as enterobacteria. It 837 is therefore assumed that both CARD and RefSeq databases are heavily biased, but not towards the 838 same genera. These biases will, naturally, affect analyses performed in this study. However, as one 839 of the most curated and widely used ARG databases, CARD is the only obvious choice for this study. 840 Likewise, RefSeq complete genomes comprise a large and well-curated database of publicly 841 available genomes and is the natural choice for this study. Both RefSeq and CARD databases are 842 generally biased towards the Enterobacteriales order but RefSeq is more skewed towards 843 Bordetella, Streptococcus, and Salmonella than CARD. On the other hand, CARD entries are 844 overrepresented by Acinetobacter, Klebsiella, and Escherichia compared to RefSeq (Supplementary 845 Figs. 7,8a). 
a

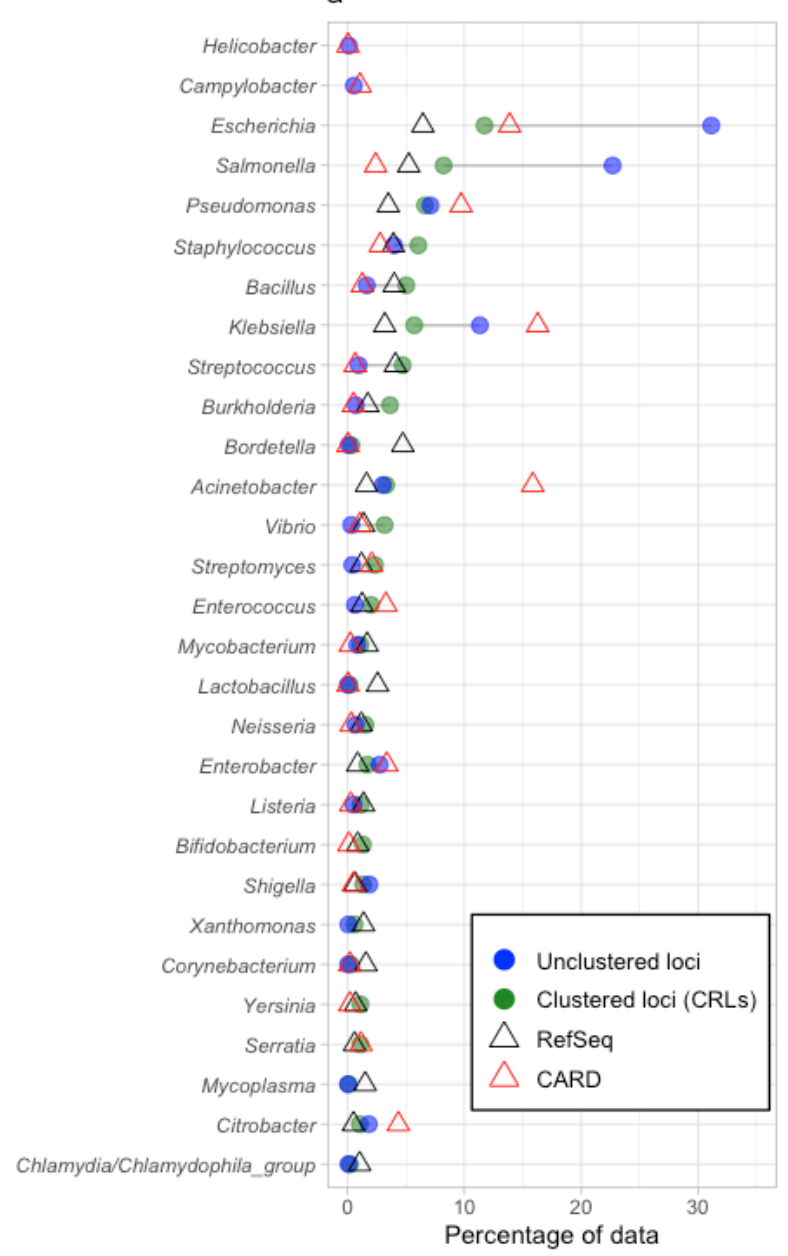

b

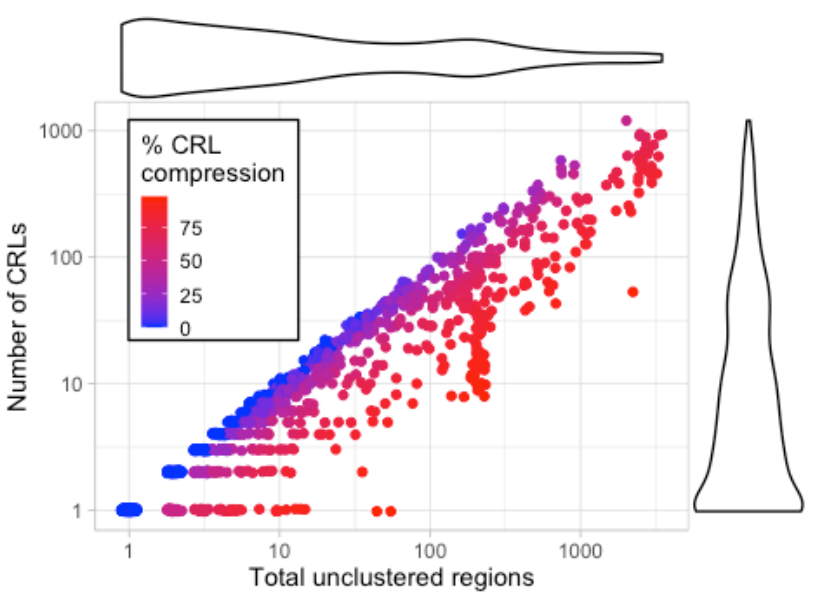

C

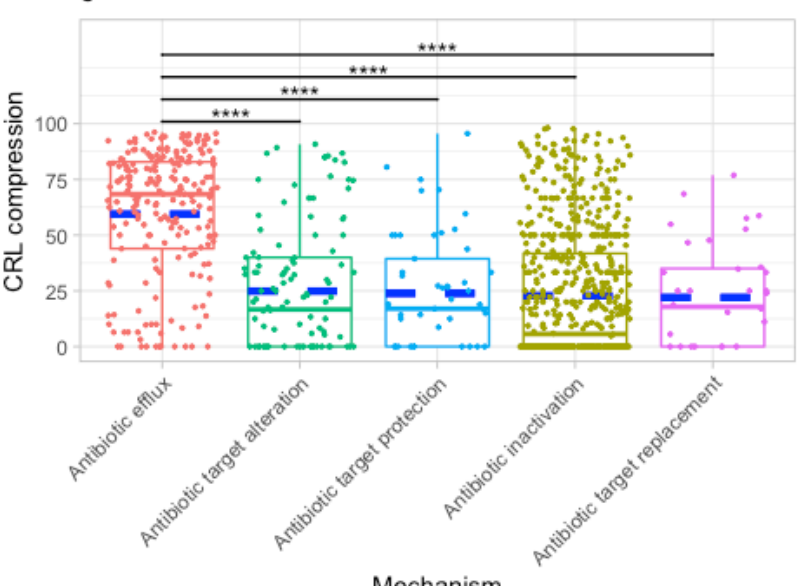

Mechanism

Supplementary Fig. 8: Effect of clustering ARG loci to CRLs. a, Database content of most abundant genera and the effect of clustering to CRLs. Clustered loci (CRLs; green circles), divided into the parent genus, generally come closer to the relative abundance of the given genus in the RefSeq complete genomes database (black triangles), than the unclustered, redundant ARG loci (blue circles). The relative abundance of genera from the CARD database are also shown (red triangles). Only genera that make up at least $1 \%$ of the total dataset of either unclustered loci, CRLs, or RefSeq complete genomes are shown. The displayed genera constitute $92.68 \%, 78.43 \%, 61.64 \%$, and $81.52 \%$ of the total datasets for unclustered loci, CRLs, RefSeq, and CARD databases, respectively. $b$, Compression effect of clustering ARG loci to CRLs. Each dot represents a single ARO category where its position on the $x$-axis indicates the number of identified unclustered loci and its position on the $y$-axis is the number of CRLs produced from clustering. The colour of the points shows the compression rate of clustering in percentage, calculated by dividing number of CRLs with the number of unclustered loci. 
Red points indicate that there are many, almost identical, ARG loci for a given ARO category, resulting in a low number of CRLs relative to the number of unclustered loci. Vice versa, blue points indicate that most of the unclustered loci are unique and clustering results in a number of CRLs that is close to the number of total unclustered loci. Violin plots are shown for both axes on the outside of the plot. Note that the position of points have been jittered very slightly to improve visualization. c, The CRL compression rate per major resistance mechanism (the very low abundance 'Reduced permeability to antibiotic' category is not shown). Each jittered point is a unique ARO CRL. Boxplot beneath CRL points show interquartile range and median as solid horizontal line. The dashed blue line indicates the mean. Whiskers extend to 1.5 * of the interquartile ranges and outliers of this range are not highlight. Difference in means of CRL compression per resistance mechanism were tested with Mann-Whitney U-test with FDR correction for multiple testing. Only significant differences are shown $(* * * * P<0.0001)$.

In order to minimize the impact of oversampling of almost identical genomes from RefSeq (e.g. $E$. coli substrains), ARG loci were clustered with USEARCH to $99 \%$ sequence similarity over at least $90 \%$ of the region length (length of ARG $+12,170 \mathrm{bp}$ in both directions). 176,688 loci with ARGs passed all filters and were clustered to 53,895 CRLs that represent 1,176 individual CARD ARO categories out of a total of 2,617 in the CARD protein homolog database (Supplementary Table 2). The missing AROs are located in bacteria that do not yet have completed genomes or are so similar to one of the 1,176 identified AROs that they were not included, since only the best ARO match per query protein was considered. Indeed, removing the initial 1,176 AROs from the CARD database and performing the analyses again resulted in 336 AROs that were not included in the main analysis (results not shown). 
Supplementary Table 2 . Of the 176,888 loci passing filters, 115,268 pass the RGI bitscore cutoff while 61,620 pass the $80 \%$ ID filter. All hits have at least $80 \%$ query coverage against the CARD ARGs.

\begin{tabular}{|l|l|l|l|}
\hline $\begin{array}{l}\text { Blastp hits against } \\
\text { CARD } \\
\left(\begin{array}{l}80 \% \text { Qcov, E-value } \\
10 \mathrm{E}-10)\end{array}\right.\end{array}$ & $\begin{array}{l}\text { DNA loci with ARG } \\
\text { hits passing filters } \\
\text { (RGI and/or ID > 80\%) }\end{array}$ & Clustered CRLs & $\begin{array}{l}\text { Total number of AROs } \\
\text { represented }\end{array}$ \\
\hline $1,341,463$ & 176,888 & 53,895 & 1,176 \\
\hline
\end{tabular}

Genetic loci with ARGs are summarized based on the genus of the strain they occur in (Supplementary Fig. 8a). The relative abundance of each genus with ARGs are compared with the relative abundance in the RefSeq complete genome database. The difference between relative abundances per genus (given in percent of total database size) are summarized, resulting in a Euclidean distance of 30.89 (373 genera) between unclustered loci and RefSeq. When clustering these loci to CRLs, the Euclidean distance of relative genera abundances compared to RefSeq abundance is reduced to 10.26 (370 genera), which shows that clustering reduces effect of oversampling of e.g. almost clonal E. coli strains. The most abundant genera, which make up at least $1 \%$ of either unclustered loci, CRLs, or RefSeq, are shown in Supplementary Fig. 8a. Almost all of the 29 genera shown are associated with either human pathogens or other anthropogenic activity (Lactobacillus, Bifidobacterium), showing that bacteria in these ecological niches are overrepresented in the both the RefSeq and the CARD database. The CARD database is even more biased towards known pathogens including Escherichia, Pseudomonas, Klebsiella, Acinetobacter, and a few others (Supplementary Fig. 8a). This bias is sure to have a major effect on ARG prediction and leads to high ARG estimates in these genera compared to others (e.g. environmental bacteria). Clustering nearly identical ARG loci to CRLs definitely helps to smoothen this skew but it cannot completely even out the biases discussed here. Furthermore, clustering to CRLs reduces the overemphasis on the human-associated genera shown in Supplementary Fig. 8a from $92.68 \%$ of the total ARG loci to $78.43 \%$, which slightly improves representation of other genera. These database biases are not surprising, since many researchers and clinicians are interested in human pathogens or closely related bacteria that can develop and transfer antimicrobial resistance. Therefore, we 
accept these biases in the present study but are aware that environmental bacteria and their potential resistance genes are underrepresented here.

918 Not all ARO categories are compressed equally in relative abundance by clustering loci to CRLS 919 (Supplementary Fig. 8b,c). Some AROs are represented only by completely unique DNA loci in the 920 unclustered dataset, resulting in a number of CRLs that is the same as the number of unclustered 921 loci (low compression rate). On the other hand, other AROs are represented by a large number of 922 nearly identical DNA loci which either stem from biased oversampling of e.g. almost clonal E. coli or 923 by clonal expansion of one or more DNA loci by HGT (high compression rate).

925 Generally, loci with efflux pump resistance determinants have a significantly higher average CRL 926 compression rate than the other functional categories (Figure 1, Supplementary Fig. 8c; P $<0.0001$ ), 927 which means that there are many almost clonal DNA loci with efflux pumps in the RefSeq complete 928 genome database. The other general functional categories do not have a significantly different CRL 929 compression rate, signifying that AROs belonging to these categories are located in more diverse 930 DNA loci than efflux pumps are. 


\section{Smoothed kernel density estimate} and ARG-MOB groupings

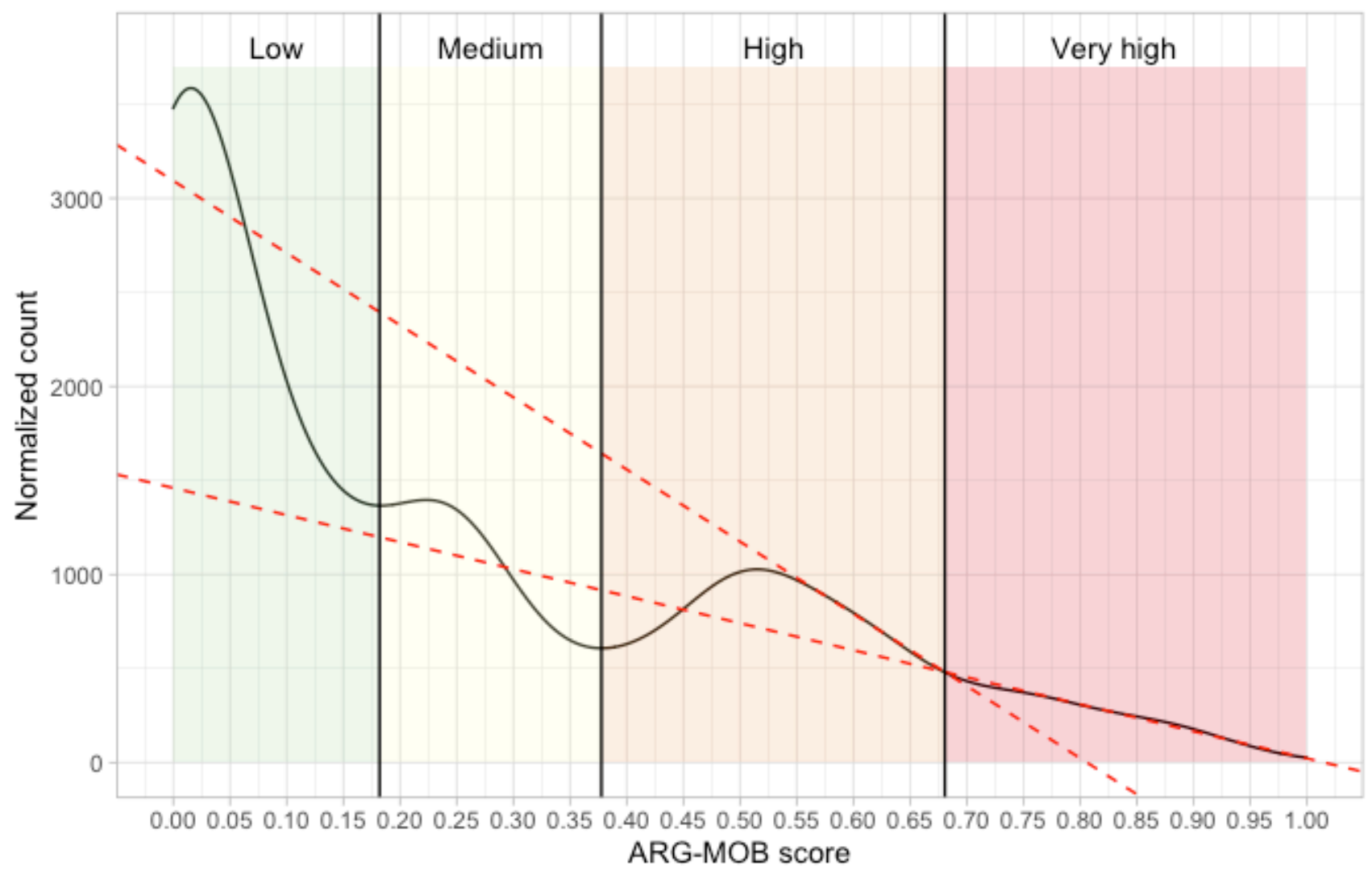

Supplementary Fig. 9. Smoothed kernel density estimates of all AROs and their ARG-MOB values. ARG-MOB values defining the categories were computed by identifying local minima in $R$. The High/Very high intersection is not found by this method. Instead, linear models were fitted to High interval 0.55-0.65 and Very high interval 0.7-1. These intervals are approximately linear. The intersection between the two models (0.685) is used as the High-Very high limit. 


\section{Supplementary Note 5: IS element families in major mechanisms}

The IS elements occurring in the proximity of putative ARGs were classified by their IS families and tested for significantly different relative abundances between resistance mechanisms (Supplementary Fig. 10). Among the 17 most abundant IS families, IS1, IS110, IS1595, IS1380, IS200/IS605, IS21, IS3, IS30, IS4, IS5, IS6, IS66, IS91, ISL3, and Tn3 occur with significantly different frequencies in $\mathrm{Al}$ and $\mathrm{AE}$ loci, with lower median frequencies in $\mathrm{AE}$. These families are therefore more active in decontextualizing putative $A I A R G$ s than $A E$, accompanying the observation that $A l$ is generally more mobilized than AE. CRLs of the ATR mechanism are significantly less associated with a lack of IS elements, which in turn means that there are more CRLs of this mechanism with IS elements in proximity. This is also shown in Figure 3 and Supplementary Fig. 11 where it is apparent that this mechanism is very often found in association with IS elements and often on plasmids.

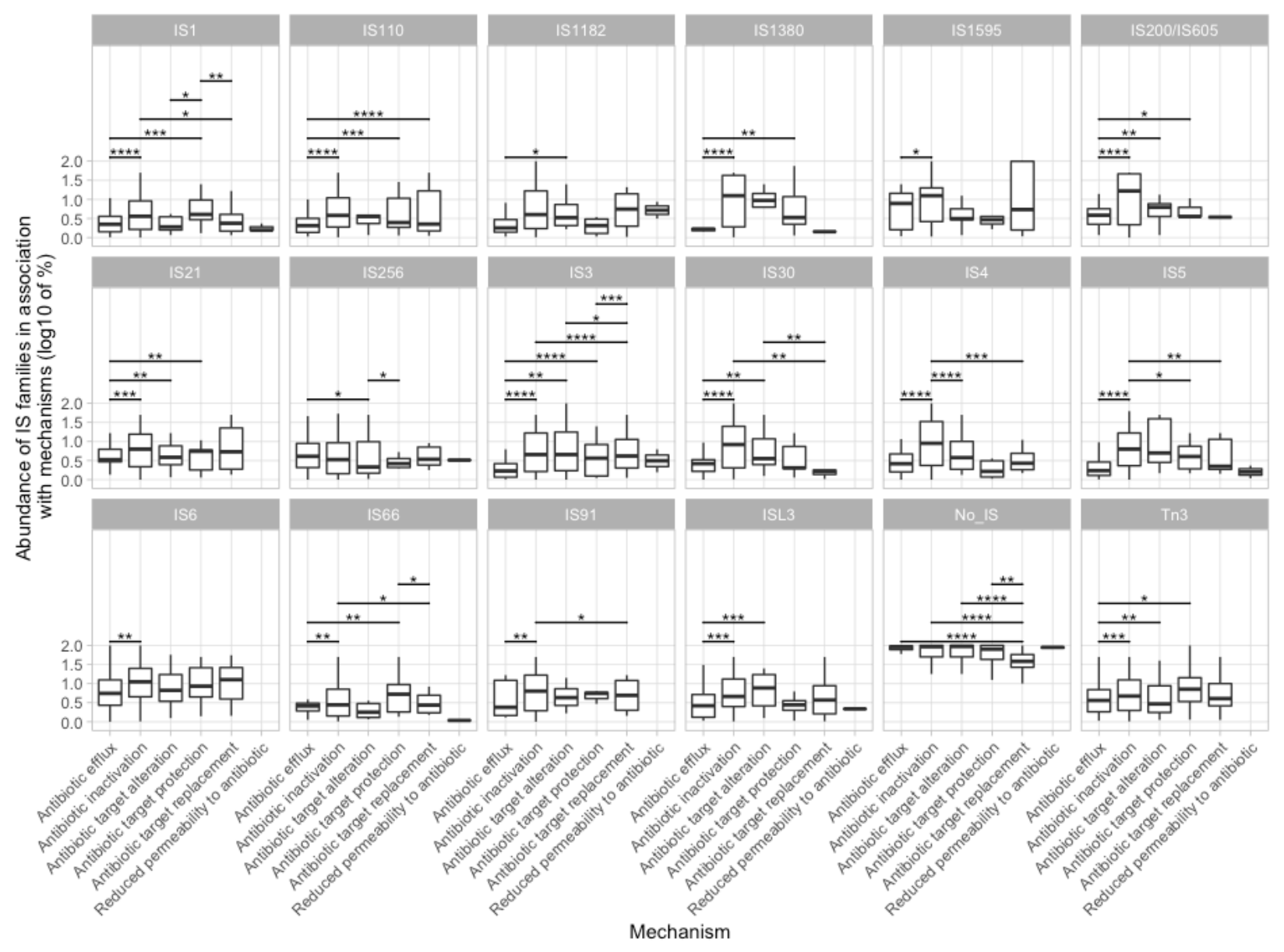


Supplementary Fig. 10. Relative abundance of 17 IS families and the lack of IS elements (No_IS) in ARO mechanisms. Only IS families with significant differences in mean value between mechanisms are shown. Log10 of relative abundance on $Y$-axes is derived from the frequency of IS families per mechanism category. Boxes indicate first and third quartiles (25\% and $75 \%$ of data) and horizontal line in box shows the median. Whiskers extend to 1.5 * of the interquartile ranges. Above boxplots, 962 bars indicate significant differences in mean between mechanisms (Mann-Whitney test with FDR correction). Only significant differences are displayed (****: $p<=0.0001)$.

\section{Supplementary Note 6: Antibiotic efflux ARGs are more loosely associated with IS elements}

966 Within the $12,170 \mathrm{bp}$ investigated in both directions of identified ARGs, the distance to nearest IS 967 element might be thought to be an indicator of how "tightly" associated a given ARG is with an IS 968 element. Likely, there are some false positive associations between ARGs and IS elements found in 969 the extremes of the $12,170 \mathrm{bp}$ maximum distance. The mean distance between ARGs and IS 970 elements per ARO is shown on the Y-axes in Supplementary Fig. 11a (mean distance per ARO is 971 calculated from unique CRLS). For $A E$, the bulk of the AROs have low IS ratios and a mean distance 972 to closest IS elements of just over $5000 \mathrm{bp}$. This is significantly different from 4 of the 5 other major 973 mechanisms (Supplementary Fig. 11b), with the exception for the very low abundance Reduced 974 permeability to antibiotic mechanism. The AE AROs that do have higher IS ratios also have shorter 975 distances to closest IS elements, which is comparable to AROs of other mechanisms that are also 976 high mobilized. For these mobilized AROs, the mean distance between ARGs and IS elements is closer to $2,500 \mathrm{bp}$. 
a

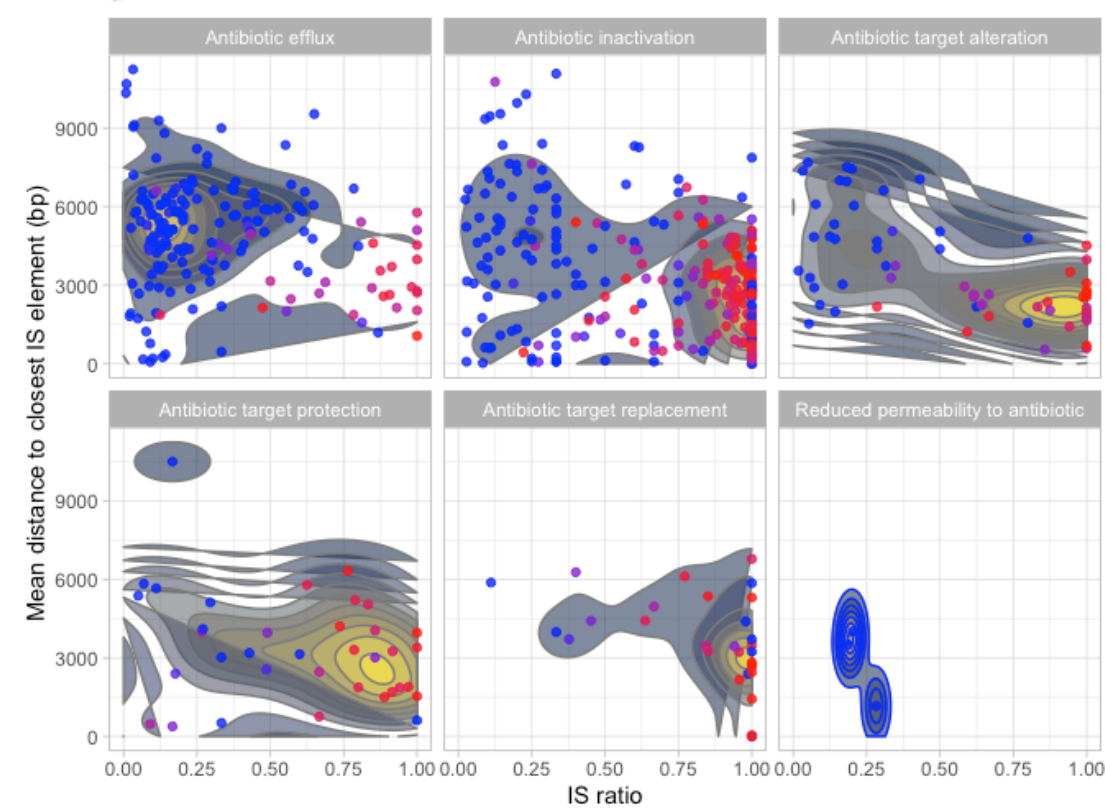

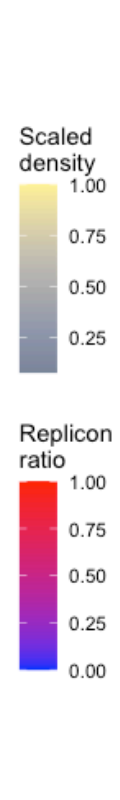

979

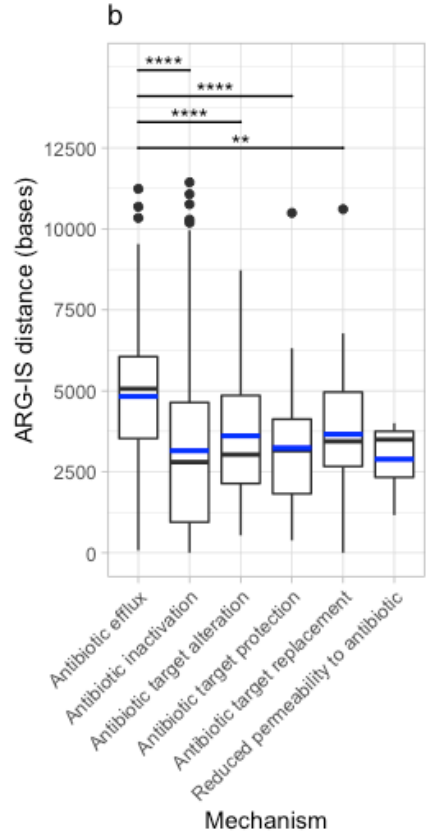

Supplementary Fig. 11: Distance between ARGs and closest IS element. a, Density plots of IS ratio against the mean distance in bases to the nearest IS element in both directions. Each point represents a unique ARO category. Plots are divided into the individual mechanisms and are colored according to the replicon ratio, where a high ratio (red) indicates that an ARO is more often found on plasmids and a low ratio (blue) indicates that an ARO is more on chromosomes. Density estimates are calculated with two-dimensional kernel density estimation, as implemented in the stat_density_2d function under the ggplot $R$ package. $b$, Boxplot of median distance (bases) between ARGs and closest IS elements. Mean is shown with blue dashed lines. Boxes indicate first and third quartiles (25\% and $75 \%$ of data) and horizontal line in box shows the median. Whiskers extend to $1.5 *$ of the interquartile ranges. Outliers are shown as black dots. Above boxplots, bars indicate significant differences in mean between mechanisms (Mann-Whitney test with FDR correction). Only significant differences are displayed (**: $p<=0.01, * * * *: p<=0.0001)$. 


\section{Supplementary Note 7: Taxonomic investigation}

995 Within Proteobacteria, Enterobacteriaceae have a higher median IS ratio than 996 Campylobacteriaceae, and Burkholderiaceae but lower than Aeromonadaceae, Pasterurellaceae and Morganellaceae (Supplementary Fig. 12). However, CRLs in Enterobacteriaceae are more often found on plasmids than for Campylobacteraceae, Moraxellaceae, Morganellaceae, Neisseriaceae, Pasteurellaceae, Pseudomonadaceae, and Burkholderiaceae (Supplementary Fig. 13; MWU test; P < 0.01), showing that many putative ARGs in Enterobacteriaceae are highly mobilized by both IS elements and plasmids.

1002 Within Enterobacteriaceae, many putative ARG loci have been mobilized both by IS elements and plasmids, especially within genera Shigella, Escherichia, Salmonella, Klebsiella, Enterobacter, and to a lesser degree Citrobacter (Supplementary Fig. 12c). Other genera in Enterobacteriaceae show lower mean mobilization degrees (Significance values in Supplementary Table 3). Enterobacteriaceae genera with highly mobilized ARGs all have members of significant importance to human health and persistent fixation of mobilized ARGs are likely a consequence of human interference with pathogenic bacteria ${ }^{3}$.

Phyla whose members are more associated with the environment, such as Actinobacteria and Bacteriodetes, have lower median IS ratios than Proteobacteria (MWU test; $\mathrm{P}<0.01$ ). However, within e.g. Firmicutes, whose IS ratio is not different from Proteobacteria, some families are also associated with human activities such as Enterococcaceae and Staphylococcaceae. These also harbour highly mobilized ARGs, while environmentally associated Firmicutes, such as Bacillaceae, barely have ARGs mobilized by either IS elements or plasmids (Supplementary Fig. 14). This exemplifies how homologs of ARGs can be found in both environmental and clinically relevant genera, but that they have been decontextualized more in the latter. 
Phyla

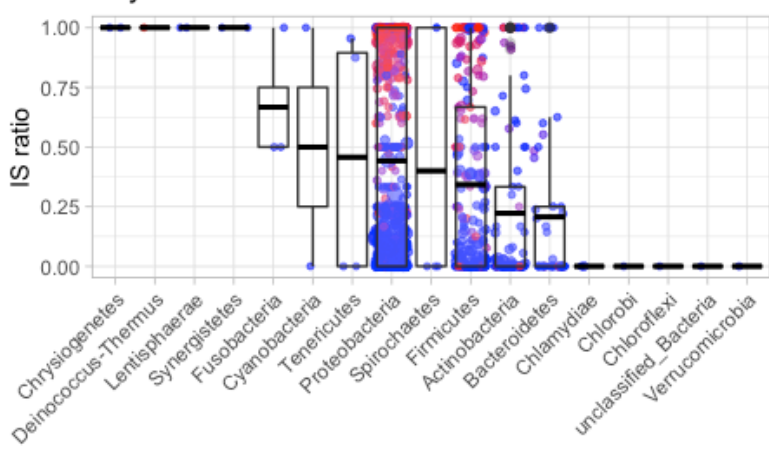

Phylum
Top 10 Proteobacteria families
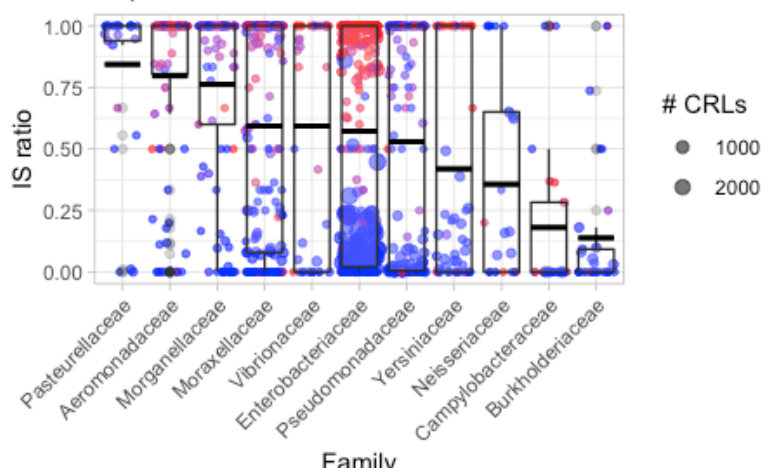

Top 15 Enterobacteriaceae genera

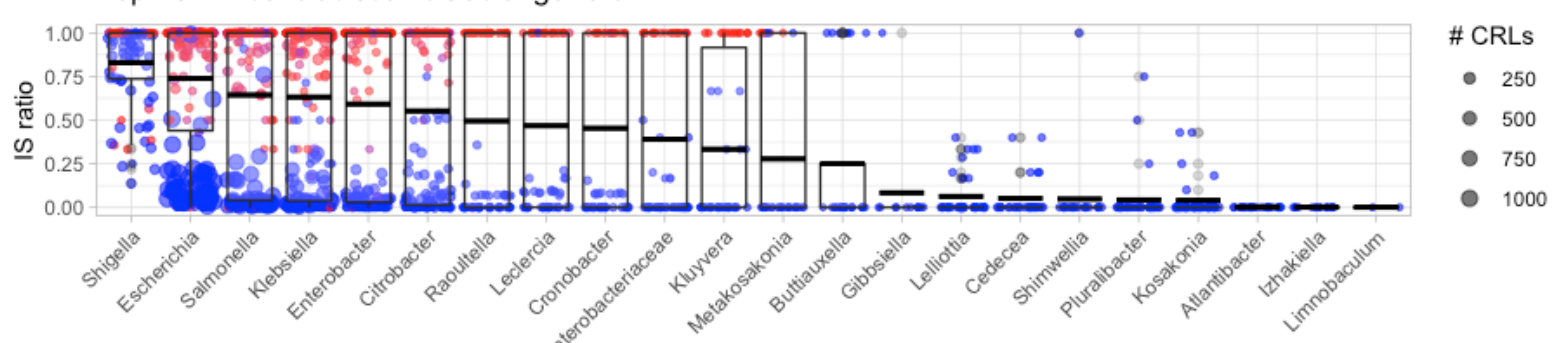

Genus

1021 Supplementary Fig. 12:. Taxonomic distribution of ARO categories. Boxplots and dots show IS ratio per taxonomic group. The size of the points indicates the number of unique CRLs in a given ARO, while the colour is the replicon ratio with highest (red) indicating more plasmid than chromosome placement of CRLS. Focus is placed on the Proteobacteria for plotting of families and genera. Boxes indicate first and third quartiles (25\% and $75 \%$ of data) and horizontal line in box shows the median. Whiskers extend to 1.5 * of the interquartile ranges. 


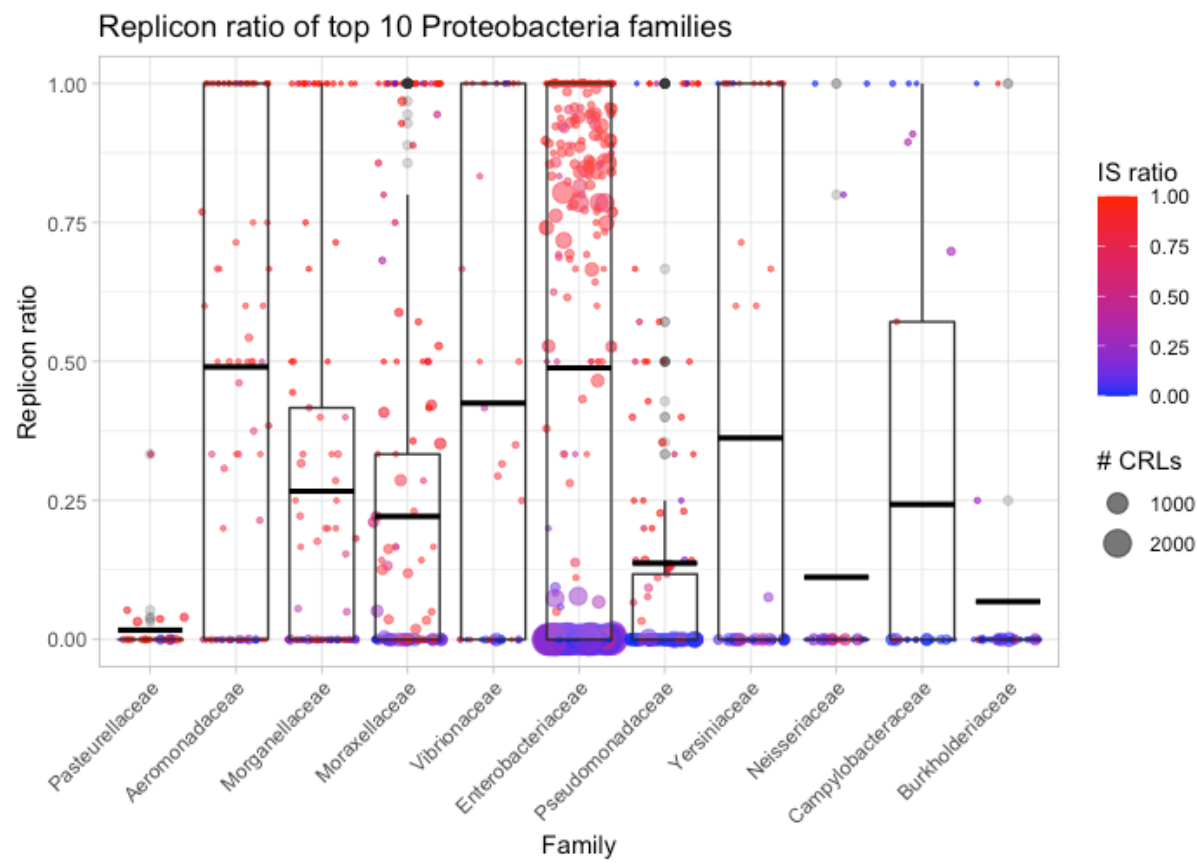

Supplementary Fig. 13. Replicon ratio of the top 10 most abundant Proteobacterial families is shown

Firmicutes families

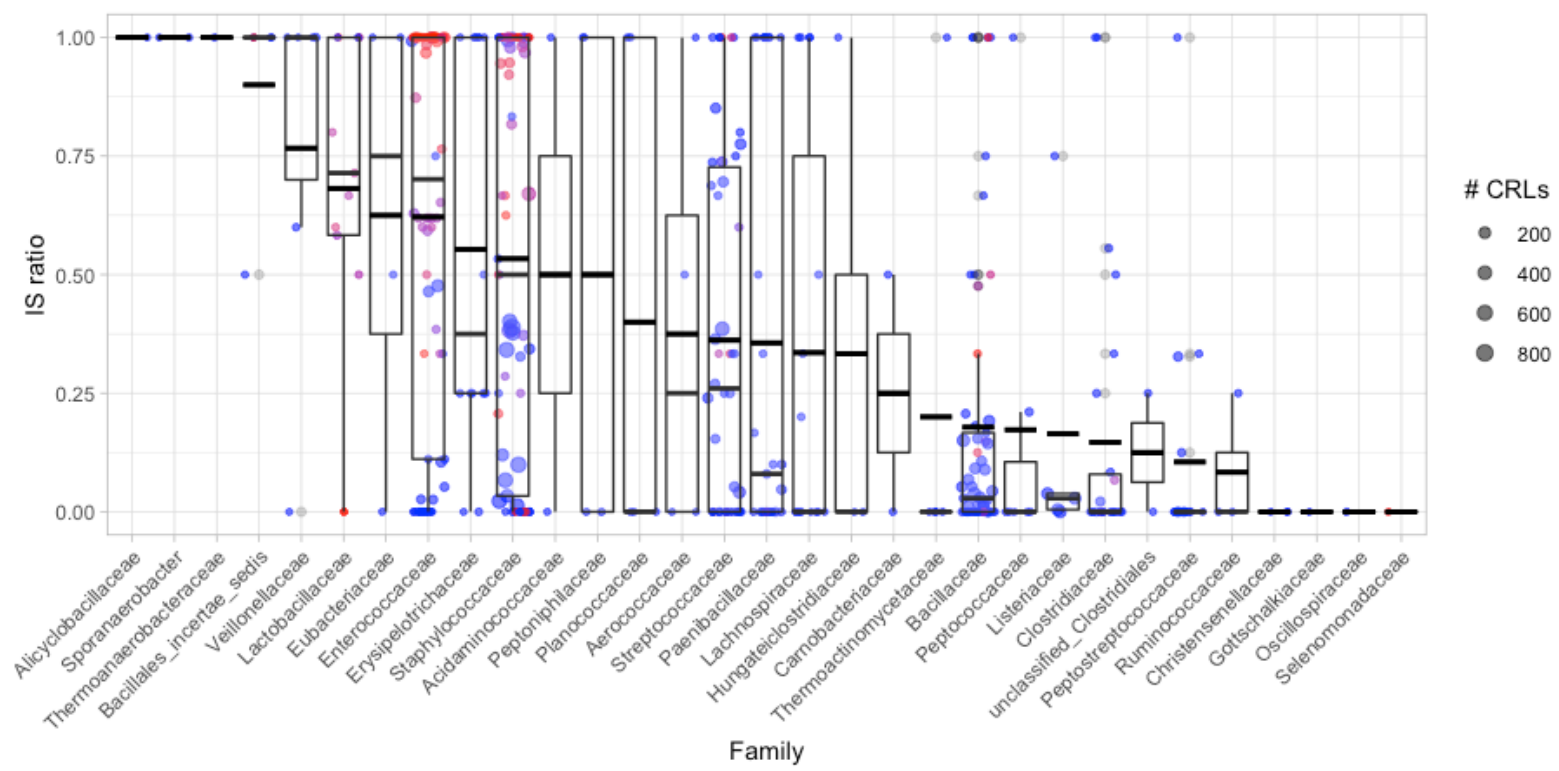

1033 Supplementary Fig. 14. Taxonomic distribution of ARO categories within the Firmicutes phylum. 1034 Boxplots and dots show IS ratio per taxonomic group. The size of the points indicates the number of unique CRLs in a given ARO, while the colour is the replicon ratio with highest (red) indicating more 
plasmid than chromosome placement of CRLs Boxes indicate first and third quartiles (25\% and 75\%

1037 of data) and horizontal line in box shows the median. Whiskers extend to 1.5 * of the interquartile 1038 ranges.

1039

1040 Supplementary Table 3. Pairwise Mann-Whitney tests for IS and Replicon ratios in 1041 Enterobacteriaceae with FDR correction for multiple testing. Only significant comparisons are listed 1042 here. Significance levels are ${ }^{*}: p<0.05 ;^{* *}: p<0.01 ; * * * p<0.001 ; * * * *: p<0.0001$

\begin{tabular}{|c|c|c|c|c|c|}
\hline Genus 1 & Genus 2 & IS p & IS sign. & Replicon p & Replicon sign. \\
\hline Cedecea & Citrobacter & 4.70E-08 & 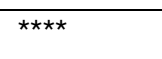 & $8.10 \mathrm{E}-06$ & 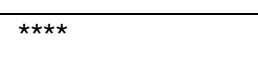 \\
\hline Cedecea & Cronobacter & 3.00E-04 & *** & 0.00026 & *** \\
\hline Cedecea & Enterobacter & 6.00E-09 & 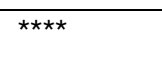 & $2.50 \mathrm{E}-07$ & 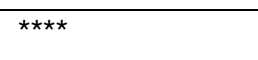 \\
\hline Cedecea & Escherichia & $1.70 \mathrm{E}-14$ & 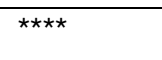 & 2.20E-09 & 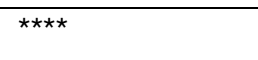 \\
\hline Cedecea & Klebsiella & $4.60 \mathrm{E}-10$ & 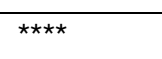 & $2.30 \mathrm{E}-08$ & 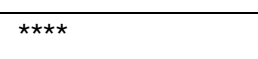 \\
\hline Cedecea & Kluyvera & 0.027 & * & 0.0078 & ** \\
\hline Cedecea & Leclercia & 3.60E-05 & 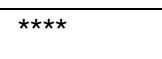 & 0.00021 & 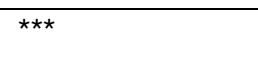 \\
\hline Cedecea & Raoultella & 3.60E-05 & $* \star \star \star \star *$ & $2.70 \mathrm{E}-05$ & 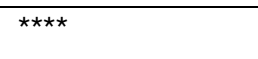 \\
\hline Cedecea & Salmonella & $5.20 \mathrm{E}-12$ & 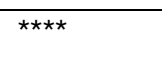 & 8.20E-08 & 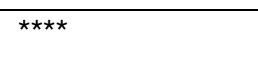 \\
\hline Cedecea & Shigella & $5.30 \mathrm{E}-14$ & 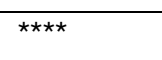 & 0.00027 & 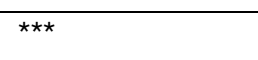 \\
\hline Cedecea & $\begin{array}{l}\text { unclassified_En } \\
\text { terobacteriacea } \\
\text { e }\end{array}$ & 0.0048 & ** & 0.00084 & 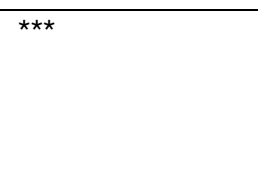 \\
\hline Citrobacter & Escherichia & 0.00023 & $* \star \star \star$ & 0.0023 & ** \\
\hline Citrobacter & Kluyvera & 0.0012 & $\star \star$ & 0.045 & * \\
\hline Citrobacter & Kosakonia & $1.50 \mathrm{E}-10$ & 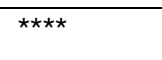 & $4.20 \mathrm{E}-07$ & 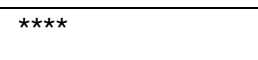 \\
\hline Citrobacter & Lelliottia & 1.60E-09 & $* \star \star \star *$ & 1.60E-07 & 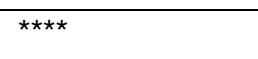 \\
\hline Citrobacter & Pluralibacter & $2.40 \mathrm{E}-11$ & 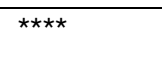 & 4.20E-07 & $\star \star \star \star \star ~$ \\
\hline Citrobacter & Shigella & 0.0098 & $\star *$ & NA & NA \\
\hline Citrobacter & $\begin{array}{l}\text { unclassified_En } \\
\text { terobacteriacea } \\
e\end{array}$ & 0.0071 & ** & NA & NA \\
\hline Cronobacter & Escherichia & 0.00082 & 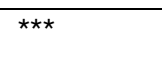 & NA & NA \\
\hline Cronobacter & Kosakonia & 1.80E-05 & $* * \star *$ & $4.20 \mathrm{E}-05$ & 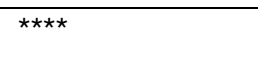 \\
\hline Cronobacter & Lelliottia & 0.00022 & $* * \star$ & 2.60E-05 & 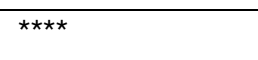 \\
\hline Cronobacter & Pluralibacter & $2.30 \mathrm{E}-06$ & 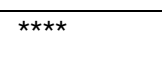 & 4.20E-05 & **** \\
\hline Cronobacter & Salmonella & 0.023 & * & NA & NA \\
\hline
\end{tabular}




\begin{tabular}{|c|c|c|c|c|c|}
\hline Cronobacter & Shigella & 0.006 & $\star \star *$ & NA & NA \\
\hline Enterobacter & Escherichia & 0.0037 & $\star \star$ & NA & NA \\
\hline Enterobacter & Kluyvera & 0.00019 & 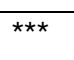 & 0.0031 & $\star \star \star *$ \\
\hline Enterobacter & Kosakonia & $1.30 \mathrm{E}-11$ & 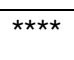 & 5.70E-09 & $\star \star * \star *$ \\
\hline Enterobacter & Lelliottia & $1.30 \mathrm{E}-10$ & 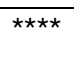 & 1.70E-09 & 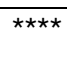 \\
\hline Enterobacter & Pluralibacter & $1.50 \mathrm{E}-12$ & 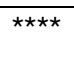 & 5.70E-09 & 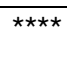 \\
\hline Enterobacter & $\begin{array}{l}\text { unclassified_En } \\
\text { terobacteriacea } \\
e\end{array}$ & 0.0015 & 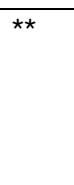 & 0.032 & * \\
\hline Escherichia & Klebsiella & 0.0041 & *夫 & NA & NA \\
\hline Escherichia & Kluyvera & $3.40 \mathrm{E}-08$ & $\overline{k \star \star \star \star}$ & 0.00022 & $\star \star \star *$ \\
\hline Escherichia & Kosakonia & $4.90 \mathrm{E}-18$ & $\star \star \star \star ~$ & $3.70 \mathrm{E}-11$ & 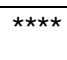 \\
\hline Escherichia & Leclercia & 0.0012 & $\star \star$ & NA & NA \\
\hline Escherichia & Lelliottia & 8.50E-18 & 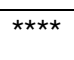 & $1.60 \mathrm{E}-11$ & 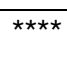 \\
\hline Escherichia & Pluralibacter & $2.70 \mathrm{E}-18$ & 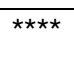 & $3.70 \mathrm{E}-11$ & 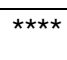 \\
\hline Escherichia & Raoultella & 0.0018 & ** & NA & NA \\
\hline Escherichia & $\begin{array}{l}\text { unclassified_En } \\
\text { terobacteriacea } \\
e\end{array}$ & $2.40 \mathrm{E}-07$ & 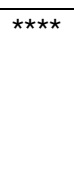 & 0.0029 & ** \\
\hline Klebsiella & Kluyvera & 6.80E-05 & 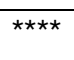 & 0.00087 & $\star \star \star$ \\
\hline Klebsiella & Kosakonia & $5.80 \mathrm{E}-13$ & 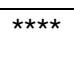 & $4.40 \mathrm{E}-10$ & 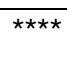 \\
\hline Klebsiella & Lelliottia & $2.30 \mathrm{E}-12$ & 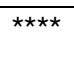 & $1.10 \mathrm{E}-10$ & $\star \star \star \star *$ \\
\hline Klebsiella & Pluralibacter & $1.10 \mathrm{E}-13$ & 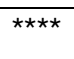 & $4.40 \mathrm{E}-10$ & 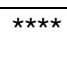 \\
\hline Klebsiella & $\begin{array}{l}\text { unclassified_En } \\
\text { terobacteriacea } \\
e\end{array}$ & 0.00053 & $\star \star \star$ & 0.0099 & $* \star$ \\
\hline Kluyvera & Kosakonia & 0.0058 & ** & 0.0025 & $\star \star$ \\
\hline Kluyvera & Leclercia & 0.034 & * & NA & NA \\
\hline Kluyvera & Lelliottia & 0.029 & * & 0.0017 & ** \\
\hline Kluyvera & Pluralibacter & 0.0017 & $\star \star$ & 0.0025 & ** \\
\hline Kluyvera & Raoultella & 0.028 & * & 0.032 & * \\
\hline Kluyvera & Salmonella & $2.50 \mathrm{E}-06$ & 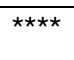 & 0.0034 & ** \\
\hline Kluyvera & Shigella & $1.20 \mathrm{E}-06$ & 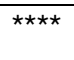 & NA & NA \\
\hline Kosakonia & Leclercia & $1.00 \mathrm{E}-06$ & $\overline{k \star \star \star}$ & 3.30E-05 & 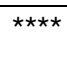 \\
\hline Kosakonia & Raoultella & $1.20 \mathrm{E}-06$ & 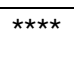 & $2.50 \mathrm{E}-06$ & 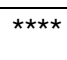 \\
\hline Kosakonia & Salmonella & $2.40 \mathrm{E}-15$ & 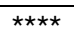 & 1.70E-09 & 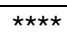 \\
\hline
\end{tabular}




\begin{tabular}{|c|c|c|c|c|c|}
\hline Kosakonia & Shigella & $1.10 \mathrm{E}-16$ & 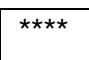 & $4.20 \mathrm{E}-05$ & 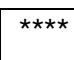 \\
\hline Kosakonia & $\begin{array}{l}\text { unclassified_En } \\
\text { terobacteriacea } \\
e\end{array}$ & 0.00061 & 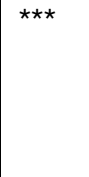 & 0.00016 & 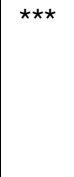 \\
\hline Leclercia & Lelliottia & 1.70E-05 & 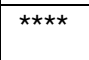 & 1.80E-05 & 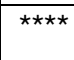 \\
\hline Leclercia & Pluralibacter & $1.10 \mathrm{E}-07$ & 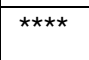 & 3.30E-05 & 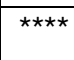 \\
\hline Leclercia & Shigella & 0.0041 & ** & NA & NA \\
\hline Lelliottia & Raoultella & $1.50 \mathrm{E}-05$ & 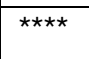 & 1.10E-06 & $\bar{k}^{* \star \star \star \star}$ \\
\hline Lelliottia & Salmonella & $2.30 \mathrm{E}-14$ & 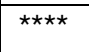 & $4.60 \mathrm{E}-10$ & 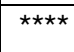 \\
\hline Lelliottia & Shigella & $2.10 \mathrm{E}-17$ & $* * * \star$ & $2.60 \mathrm{E}-05$ & 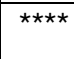 \\
\hline Lelliottia & $\begin{array}{l}\text { unclassified_En } \\
\text { terobacteriacea } \\
e\end{array}$ & 0.0037 & $* *$ & 8.90E-05 & 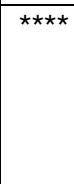 \\
\hline Pluralibacter & Raoultella & $1.60 \mathrm{E}-07$ & 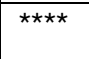 & $2.50 \mathrm{E}-06$ & 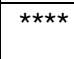 \\
\hline Pluralibacter & Salmonella & $3.00 \mathrm{E}-16$ & 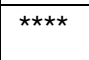 & 1.70E-09 & $* * \star *$ \\
\hline Pluralibacter & Shigella & $2.00 \mathrm{E}-16$ & 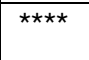 & $4.20 \mathrm{E}-05$ & $\bar{*}^{* \star \star \star \star}$ \\
\hline Pluralibacter & $\begin{array}{l}\text { unclassified_En } \\
\text { terobacteriacea } \\
e\end{array}$ & 0.00015 & 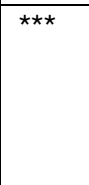 & 0.00016 & 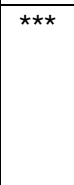 \\
\hline Raoultella & Shigella & 0.02 & * & NA & NA \\
\hline Salmonella & $\begin{array}{l}\text { unclassified_En } \\
\text { terobacteriacea } \\
e\end{array}$ & $2.40 \mathrm{E}-05$ & $* * * *$ & 0.043 & * \\
\hline Shigella & $\begin{array}{l}\text { unclassified_En } \\
\text { terobacteriacea } \\
e\end{array}$ & 2.80E-05 & 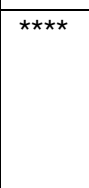 & NA & NA \\
\hline Citrobacter & Klebsiella & NA & NA & 0.011 & * \\
\hline Enterobacter & Shigella & NA & NA & 0.011 & * \\
\hline Escherichia & Salmonella & NA & NA & 0.036 & * \\
\hline Escherichia & Shigella & NA & NA & 0.00026 & $* \star \star$ \\
\hline Klebsiella & Shigella & NA & NA & 0.0016 & $* *$ \\
\hline Salmonella & Shigella & NA & NA & 0.016 & * \\
\hline
\end{tabular}


Supplementary Note 8: Supplementary information to integron analyses
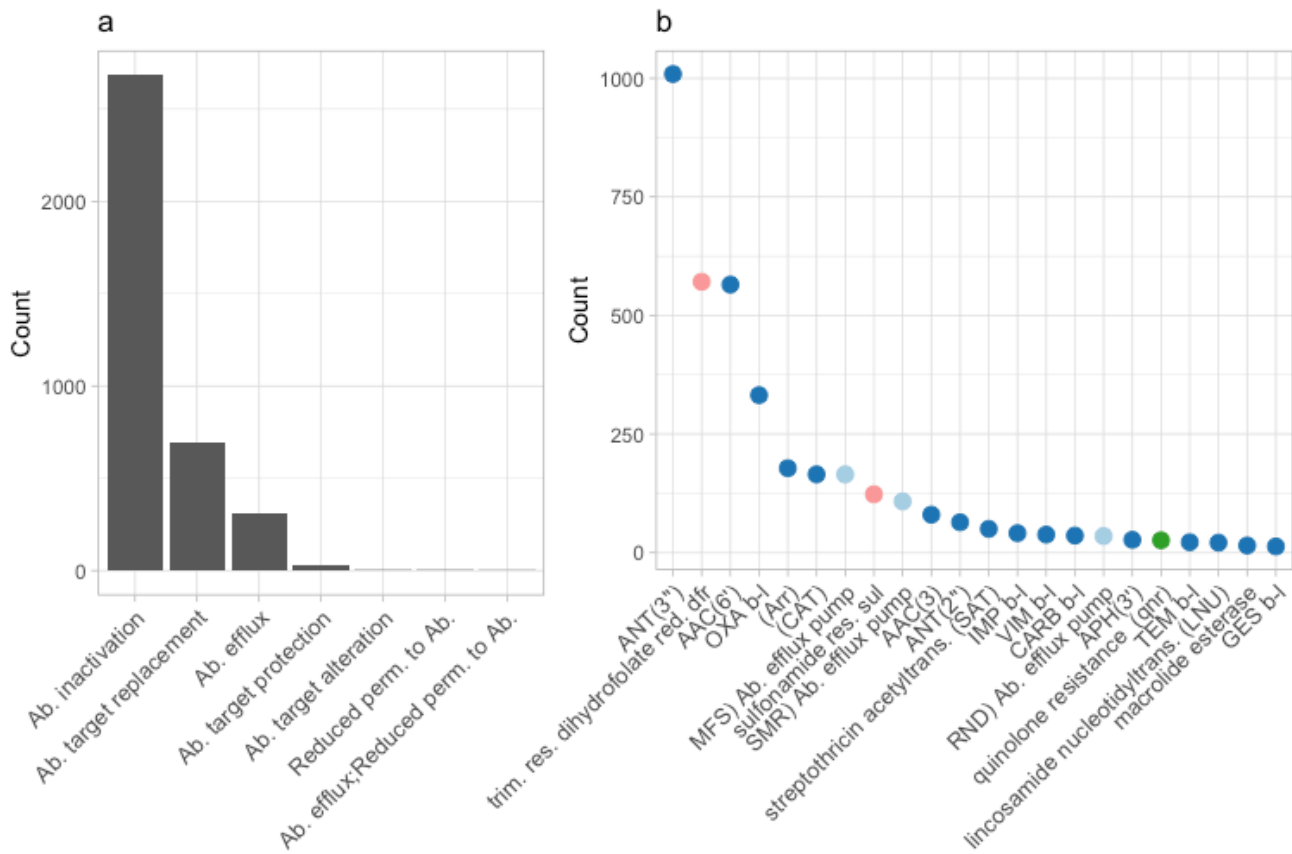
Mechanism
- $\mathrm{Ab}$. inactivation (Al)
- Ab. target replacement (ATR)
- Ab. efflux (AE)
- Ab. target protection (ATP)

Supplementary Fig. 15: ARGs associated with integrons. a, Major mechanisms associated with integrons. The count is in unique CRLs belonging to each mechanism. $b, C R L s$ where the identified $A R G$ is inserted in an integron, summarized by submechanism category. Each point can include multiple ARO categories. Only submechanisms with more than 10 CRLs are shown.

The 5 ANT AROs (aadA, aadA2, aadA5, ANT(3")-Ila, and ANT(2")-la) shown in Supplementary

1054 Table 4 all display integron associations in at least $54.24 \%$ of their total CRL occurrences. Similarly, the aminoglycoside acetyltransferase $A A C\left(6^{\prime}\right)$ AROs $\left(A A C\left(6^{\prime}\right)-I b-c r, A A C\left(6^{\prime}\right)-I b 10, A A C\left(6^{\prime}\right)-I b 7\right.$, and $\left.A A C\left(6^{\prime}\right)-I b 9\right)$ are here mostly found in integrons, agreeing with previous description of this class of $\mathrm{ARGs}^{4}$. The sulfonamide resistance sul ARO (3000410), which is canonically associated with Class 1 integrons, are actually only found in association with integrons in $10.49 \%$ of the total identified CRL occurrences ( $n=1,173$ ), while it is located outside of integrons in $89.51 \%$ of its CRLs. 
1061 Supplementary Table 4. The top 20 AROs associated with integrons.

\begin{tabular}{|c|c|c|c|c|c|c|}
\hline Gene & ARO & Mechanism & $\begin{array}{l}\text { Submechan } \\
\text { ism }\end{array}$ & $\begin{array}{l}\text { Total CRL } \\
\text { occurrence } \\
\text { s }\end{array}$ & $\begin{array}{l}\text { CRLs } \\
\text { associated } \\
\text { with an } \\
\text { integron (\% } \\
\text { of ARO } \\
\text { total) }\end{array}$ & $\begin{array}{l}\text { Percentage } \\
\text { of total } \\
\text { integron } \\
\text { occurrence } \\
\text { s }\end{array}$ \\
\hline $\operatorname{aad} A$ & 3002601 & $\begin{array}{l}\text { Antibiotic } \\
\text { inactivation }\end{array}$ & ANT(3'') & 429 & $370(86.2 \%)$ & $13.24 \%$ \\
\hline $\operatorname{aad} A 2$ & 3002602 & $\begin{array}{l}\text { Antibiotic } \\
\text { inactivation }\end{array}$ & ANT(3' $)$ & 399 & 346 (86.7\%) & $12.38 \%$ \\
\hline $\begin{array}{l}A A C\left(6^{\prime}\right)-I b- \\
c r\end{array}$ & 3002547 & $\begin{array}{l}\text { Antibiotic } \\
\text { inactivation }\end{array}$ & $\mathrm{AAC}\left(6^{\prime}\right)$ & 224 & 224 (100\%) & $8.01 \%$ \\
\hline dfrA12 & 3002858 & $\begin{array}{l}\text { Antibiotic } \\
\text { target } \\
\text { replacement }\end{array}$ & $\begin{array}{l}\text { trimethoprim } \\
\text { resistant } \\
\text { dihydrofolat } \\
\text { e reductase } \\
\text { dfr }\end{array}$ & 221 & $221(100 \%)$ & $7.91 \%$ \\
\hline$O X A-1$ & 3001396 & $\begin{array}{l}\text { Antibiotic } \\
\text { inactivation }\end{array}$ & $\begin{array}{l}\text { OXA beta- } \\
\text { lactamase }\end{array}$ & 277 & 177 (63.9\%) & $6.33 \%$ \\
\hline dfrA14 & 3002859 & $\begin{array}{l}\text { Antibiotic } \\
\text { target } \\
\text { replacement }\end{array}$ & $\begin{array}{l}\text { trimethoprim } \\
\text { resistant } \\
\text { dihydrofolat } \\
\text { e reductase } \\
\text { dfr }\end{array}$ & 166 & $138(83.1 \%)$ & $4.94 \%$ \\
\hline aadA5 & 3002605 & $\begin{array}{l}\text { Antibiotic } \\
\text { inactivation }\end{array}$ & ANT(3'') & 141 & 138 (97.9\%) & $4.94 \%$ \\
\hline$A A C\left(6^{\prime}\right)-I b 10$ & 3002581 & $\begin{array}{l}\text { Antibiotic } \\
\text { inactivation }\end{array}$ & $\mathrm{AAC}\left(6^{\prime}\right)$ & 144 & 135 (93.7\%) & $4.83 \%$ \\
\hline arr-3 & 3002848 & $\begin{array}{l}\text { Antibiotic } \\
\text { inactivation }\end{array}$ & $\begin{array}{l}\text { rifampin } \\
\text { ADP- } \\
\text { ribosyltransf } \\
\text { erase (Arr) }\end{array}$ & 126 & 125 (99.2\%) & $4.47 \%$ \\
\hline sul1 & 3000410 & $\begin{array}{l}\text { Antibiotic } \\
\text { target } \\
\text { replacement }\end{array}$ & $\begin{array}{l}\text { sulfonamide } \\
\text { resistant sul }\end{array}$ & 1173 & 123 (10.5\%) & $4.40 \%$ \\
\hline
\end{tabular}




\begin{tabular}{|c|c|c|c|c|c|c|}
\hline dfrA1 & 3002854 & $\begin{array}{l}\text { Antibiotic } \\
\text { target } \\
\text { replacement }\end{array}$ & $\begin{array}{l}\text { trimethoprim } \\
\text { resistant } \\
\text { dihydrofolat } \\
\text { e reductase } \\
\text { dfr }\end{array}$ & 134 & $123(91.8 \%)$ & $4.40 \%$ \\
\hline$q a c H$ & 3003836 & $\begin{array}{l}\text { Antibiotic } \\
\text { efflux }\end{array}$ & $\begin{array}{l}\text { small } \\
\text { multidrug } \\
\text { resistance } \\
\text { (SMR) } \\
\text { antibiotic } \\
\text { efflux pump }\end{array}$ & 109 & 108 (99.1\%) & $3.86 \%$ \\
\hline catB3 & 3002676 & $\begin{array}{l}\text { Antibiotic } \\
\text { inactivation }\end{array}$ & $\begin{array}{l}\text { chloramphe } \\
\text { nicol } \\
\text { acetyltransfe } \\
\text { rase (CAT) }\end{array}$ & 101 & 100 (99\%) & $3.58 \%$ \\
\hline$c m I A 1$ & 3002693 & $\begin{array}{l}\text { Antibiotic } \\
\text { efflux }\end{array}$ & $\begin{array}{l}\text { major } \\
\text { facilitator } \\
\text { superfamily } \\
\text { (MFS) } \\
\text { antibiotic } \\
\text { efflux pump }\end{array}$ & 84 & 84 (100\%) & $3.01 \%$ \\
\hline$A A C\left(6^{\prime}\right)-I b 9$ & 3002580 & $\begin{array}{l}\text { Antibiotic } \\
\text { inactivation }\end{array}$ & $\operatorname{AAC}\left(6^{\prime}\right)$ & 98 & 74 (75.5\%) & $2.65 \%$ \\
\hline ANT(3')-Ila & 3004089 & $\begin{array}{l}\text { Antibiotic } \\
\text { inactivation }\end{array}$ & ANT(3' $)$ & 94 & $67(71.3 \%)$ & $2.40 \%$ \\
\hline OXA-9 & 3001404 & $\begin{array}{l}\text { Antibiotic } \\
\text { inactivation }\end{array}$ & $\begin{array}{l}\text { OXA beta- } \\
\text { lactamase }\end{array}$ & 67 & 66 (98.5\%) & $2.36 \%$ \\
\hline$A N T\left(2^{\prime \prime}\right)-l a$ & 3000230 & $\begin{array}{l}\text { Antibiotic } \\
\text { inactivation }\end{array}$ & ANT(2' $)$ & 118 & 64 (54.2\%) & $2.29 \%$ \\
\hline$A A C\left(6^{\prime}\right)-I b 7$ & 3002578 & $\begin{array}{l}\text { Antibiotic } \\
\text { inactivation }\end{array}$ & $\mathrm{AAC}\left(6^{\prime}\right)$ & 81 & $58(71.6 \%)$ & $2.08 \%$ \\
\hline cmIA5 & 3002695 & $\begin{array}{l}\text { Antibiotic } \\
\text { efflux }\end{array}$ & $\begin{array}{l}\text { major } \\
\text { facilitator } \\
\text { superfamily } \\
\text { (MFS) } \\
\text { antibiotic } \\
\text { efflux pump }\end{array}$ & 58 & $54(93.1 \%)$ & $1.93 \%$ \\
\hline
\end{tabular}




\section{Supplementary Note 9: Pearson correlation coefficient analyses for major mechanisms}

Per major mechanism, Pearson correlation coefficients were also calculated pairwise between each of the four MOB parameters (Supplementary Figs. 16-20). For all mechanisms, IS and Replicon ratios correlate with similar correlation coefficients. ARG-IS association likewise correlates with ARGintegron association, except for the ATA mechanism. For mechanisms Al and AE, all correlations are positive and significant. For ATR, the Simpson index correlates with neither IS nor integron ratio. For ATA and ATP, four and two correlations were not significant, respectively. Numerically, AE CRLs are most often found on chromosomes with no association with IS elements or integrons (Supplementary Fig. 21). Contrary to this, Al and ATR CRLs are very often found on plasmids in association with both IS elements and integrons. Overall, there is also an evident association between integrons and IS elements and integrons without IS elements is a rare combination.

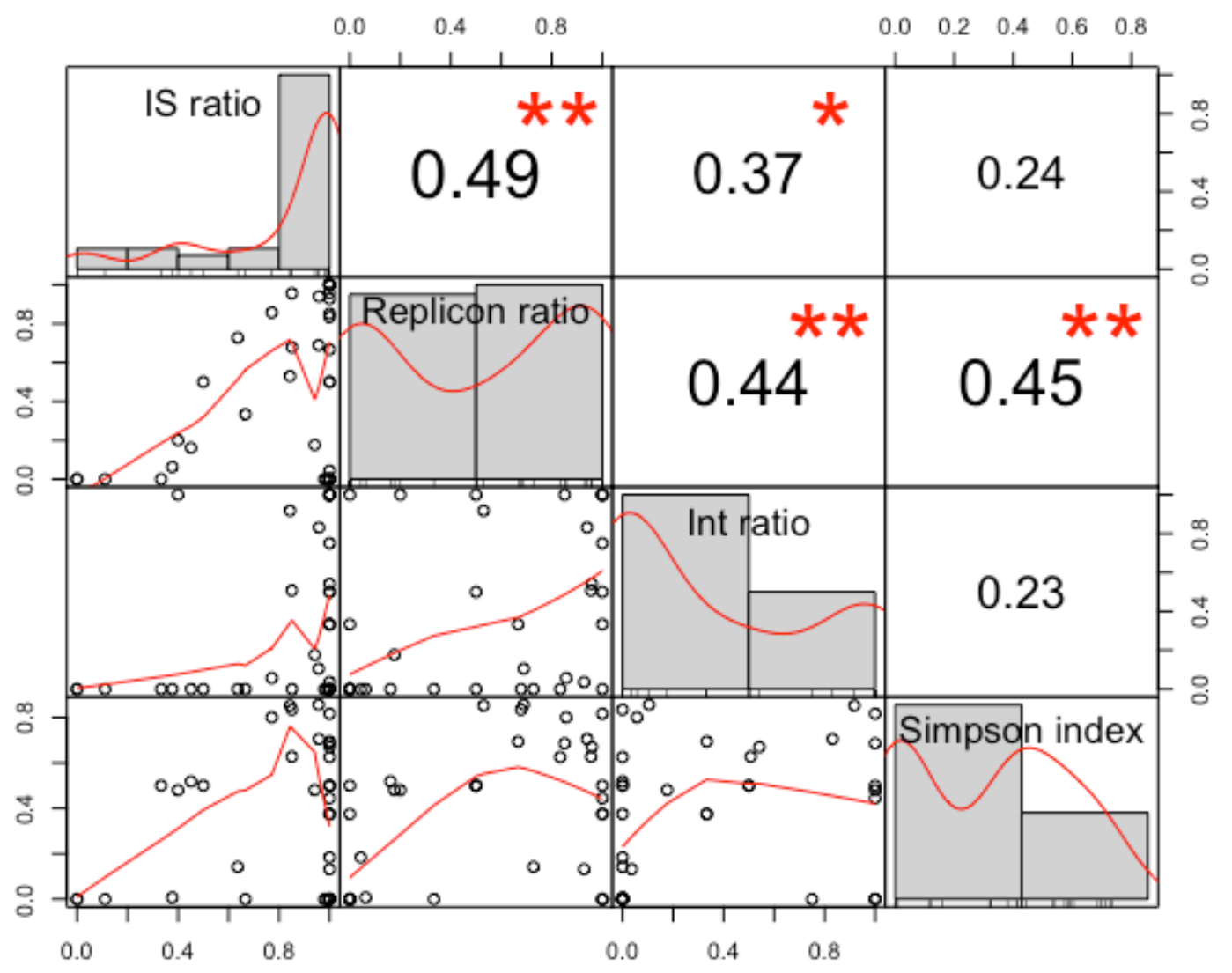




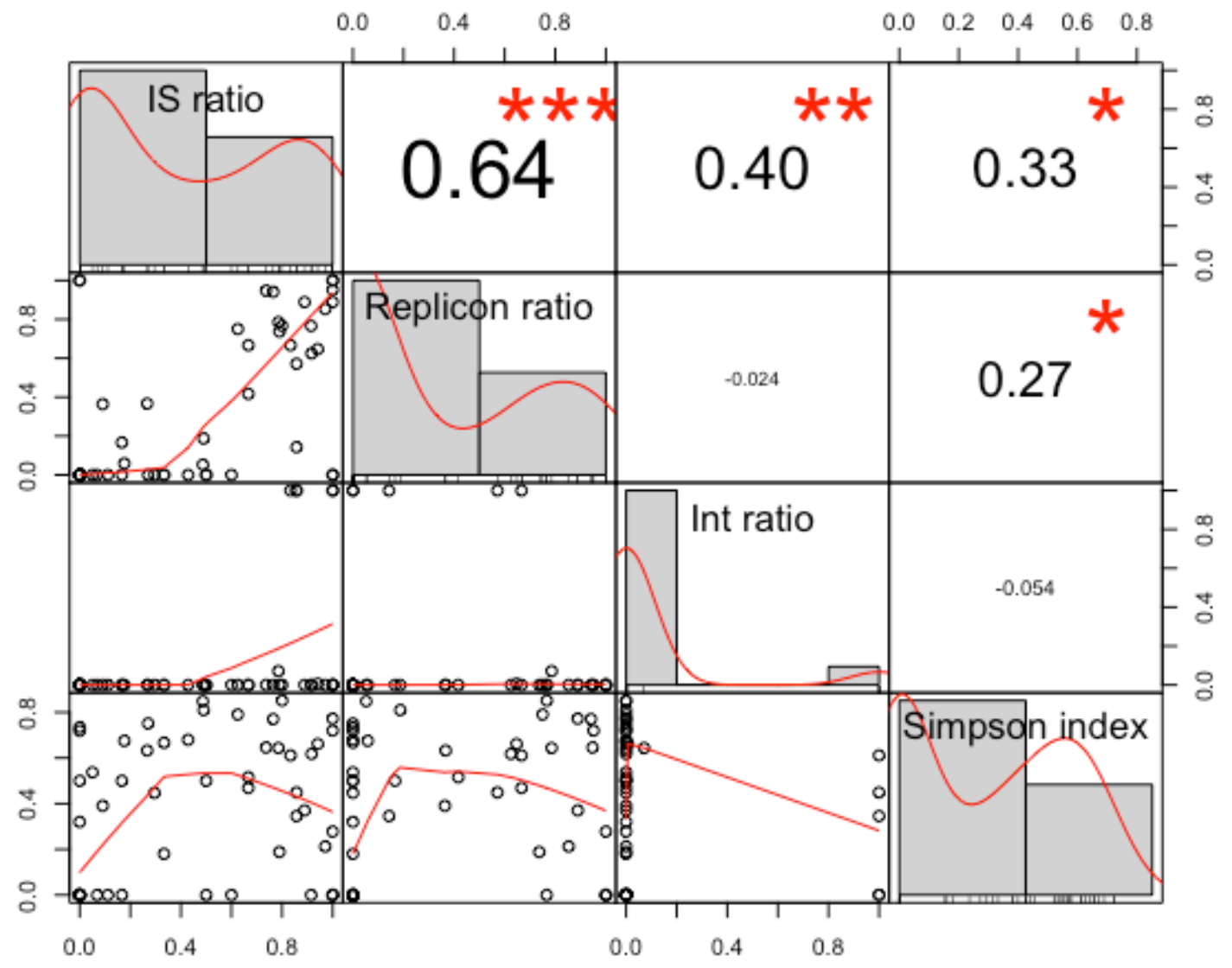




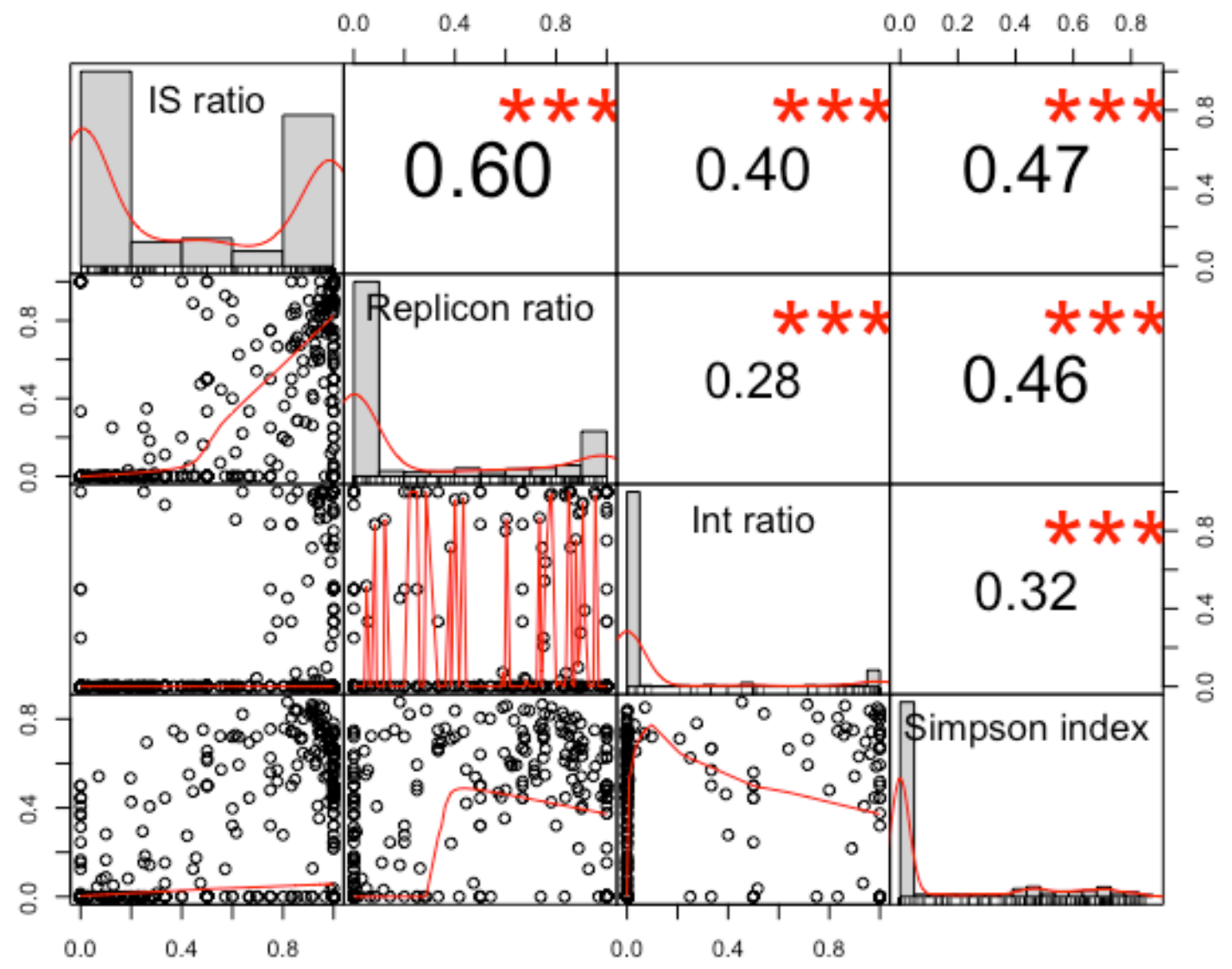




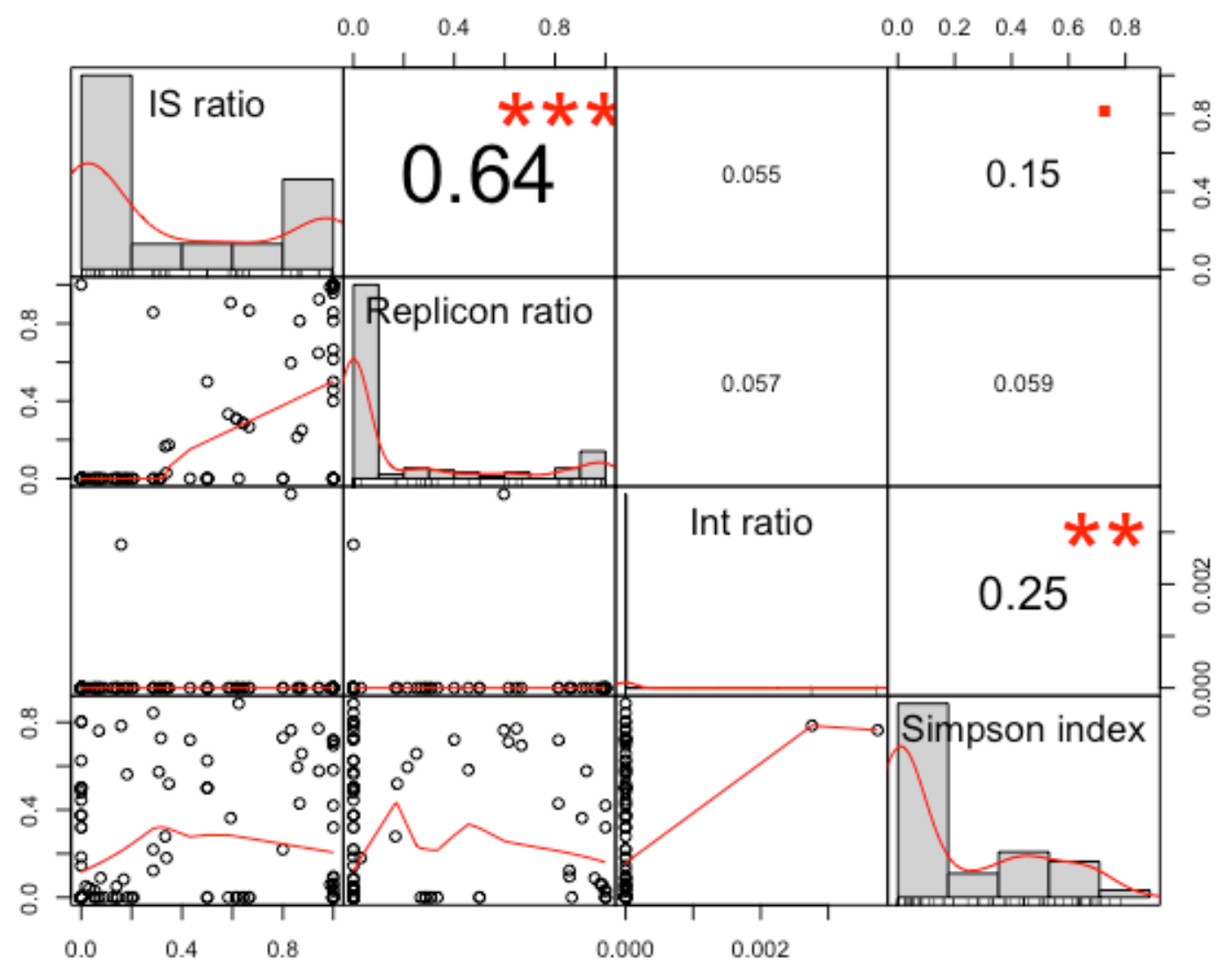




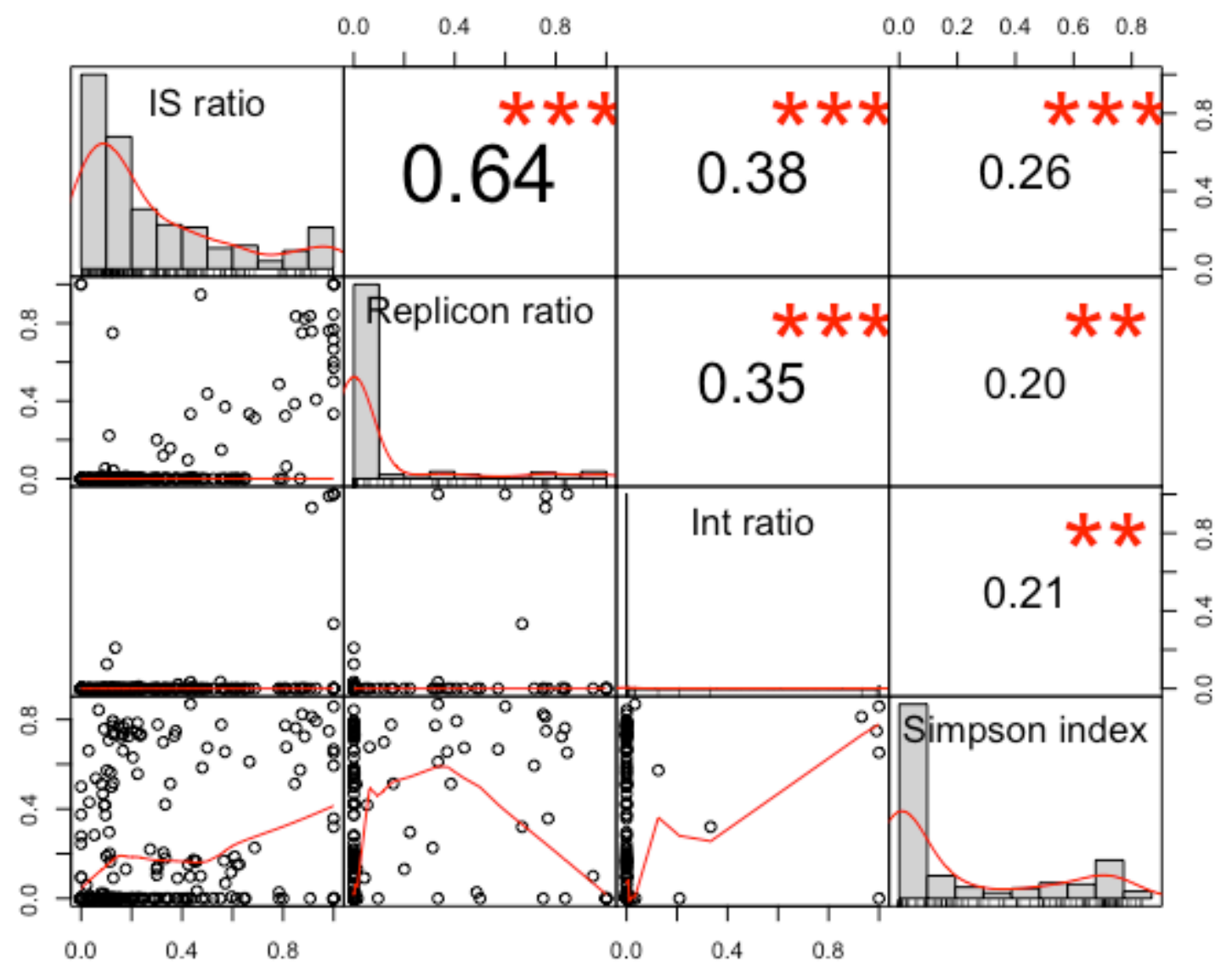



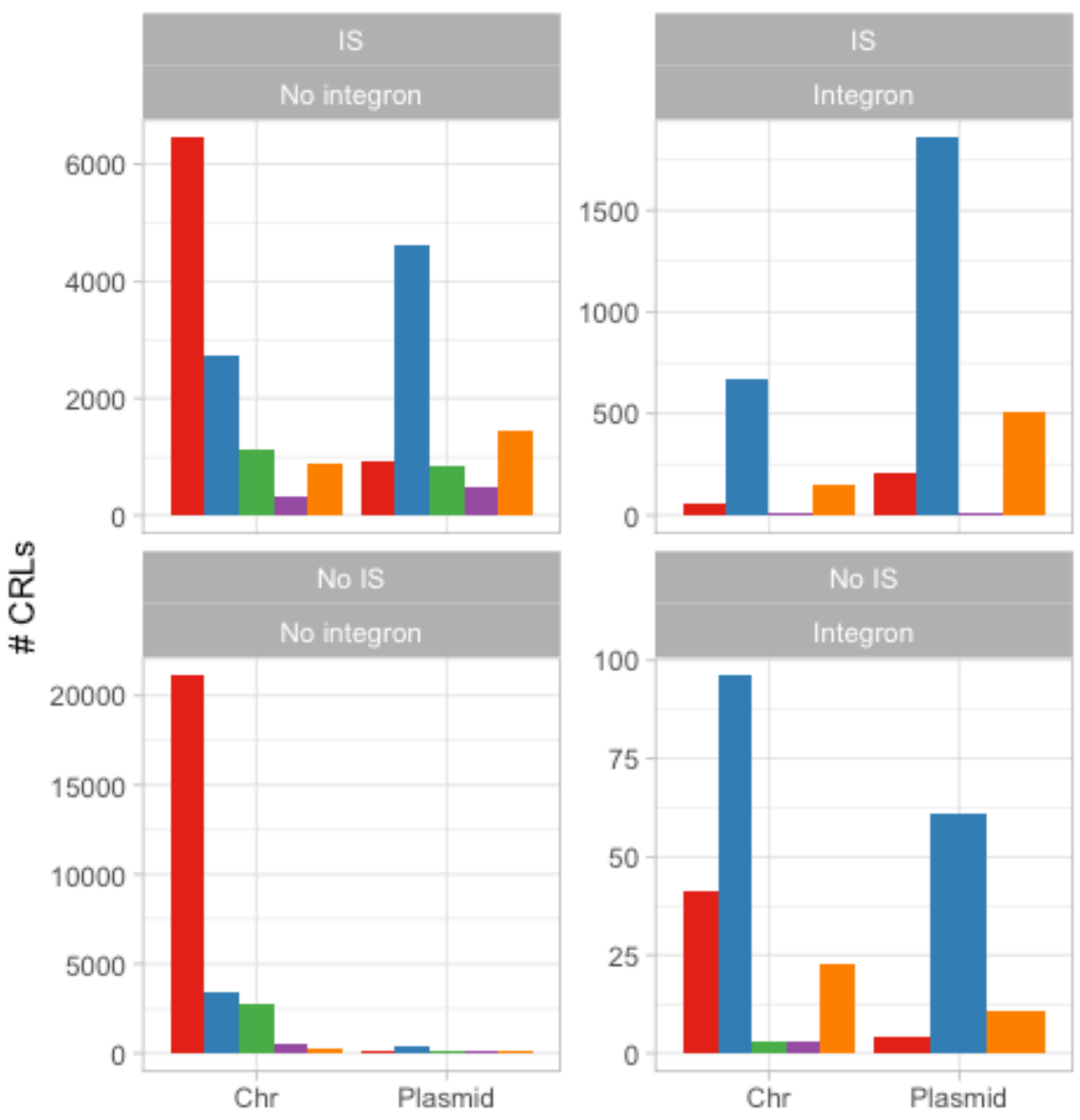

\section{Mechanism}

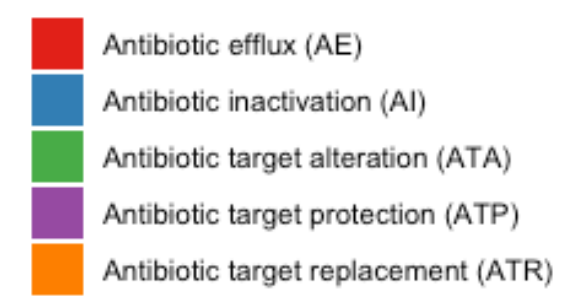

Supplementary fig. 21: Counts of CRLS separated by replicon type, and IS-and integron association.

Bars are colored by resistance mechanism. Y-axis scales are different between each subplot.

\section{Supplementary Note 10: Some AROs are highly divergent in mobilization}

1089 The mean IS and Replicon ratios per ARO are calculated across all genera they are found in. 1090 However, upon closer scrutiny of AROs per genus it becomes obvious that some AROs have a high 1091 spread from their IS and Replicon ratio means. For Supplementary Fig. 22, the genus-specific ratios 1092 were calculated per ARO and their difference from the mean global ARO ratio was calculated per genus. For each ARO, the summed positive and negative differences (for each genus with a given ARO) are shown below. A positive difference from the ARO mean indicates that there are genera in which ARGs of the given ARO are more mobilized than the ARO mean. Vice versa, a negative 
summed difference from the mean shows that ARGs of a given ARO are less mobilized in some genera than the ARO mean.

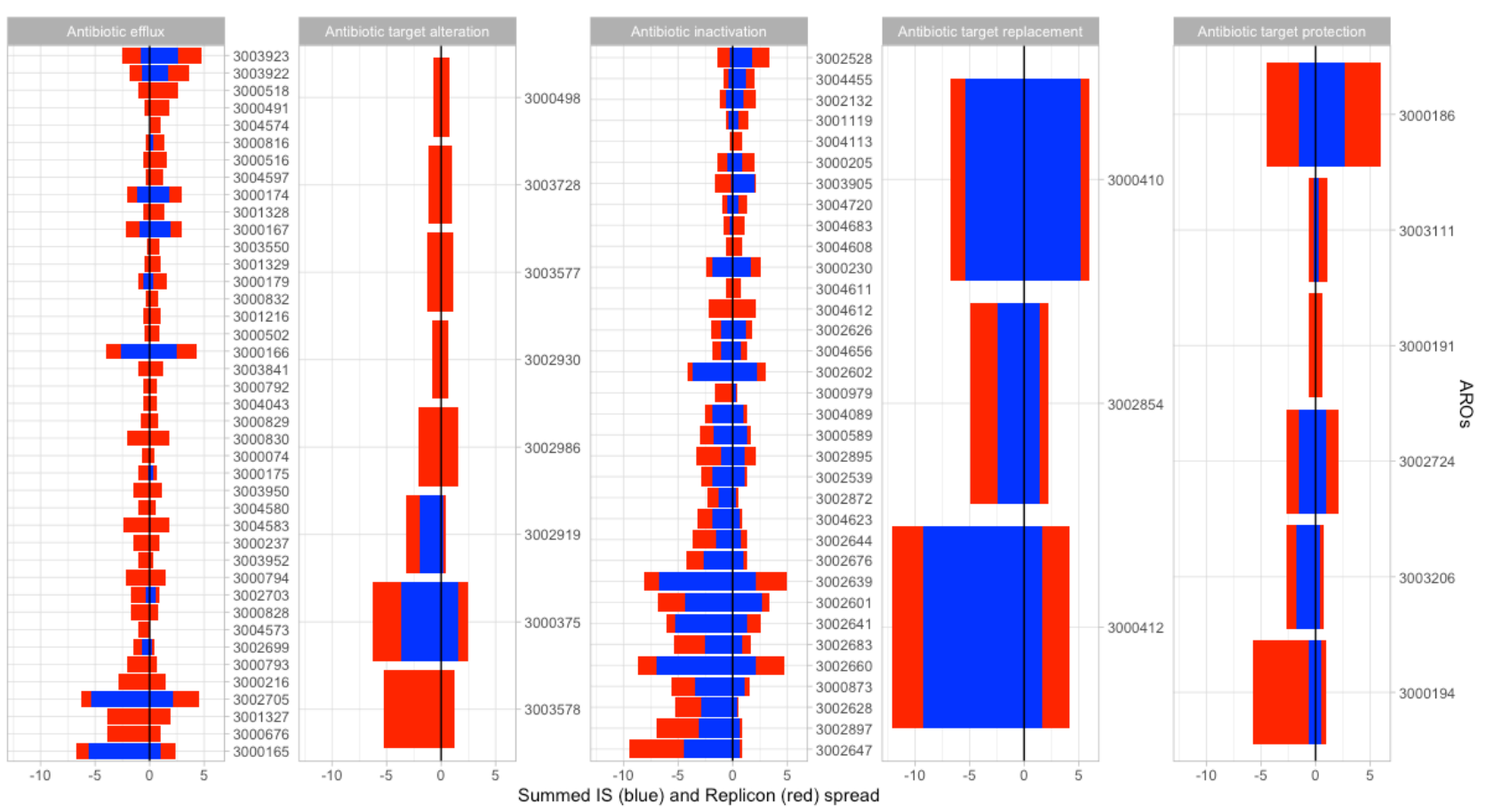

Supplementary Fig. 22: Summed spread from mean IS and Replicon ratios from the ARO means. Differences from the mean per ARO were summed by the genera in which the AROs were identified. A positive summed difference from ARO mean indicates that some genera have more mobilized ARGs of a given ARO than the ARO mean. Mobilization by both IS elements (left) and plasmids (right) are shown. Before calculating the genus-specific IS and Replicon ratio per ARO, genus:ARO combinations with only 1 occurrence in the dataset were filtered, since they are a source of noise in this context. Only AROs with a summed negative plus positive spread from mean of at least 1 are shown.

From the same dataset, genera are plotted with their summed spread from the mean of all AROs found within the respective genera (Supplementary Fig. 23). Especially Al AROs in Shigella are highly mobilized by IS elements compared to the ARO means. Interestingly, the AI AROs are not very mobilized by plasmids in Shigella, indicating that AI ARGs often associated with IS elements in Shigella but mostly on chromosomes. Also worth noting, Proteus, Pseudomonas, Morganella, Acinetobacter, and a few other genera have large negative summed Replicon spreads from mean, 
indicating that chromosomes in these may act as reservoirs for yet unmobilized ARGs. On the other

1116 hand, these putative and potential ARGs are not decontextualized and will likely occur as false-

1117 positive resistance genes in studies applying (q)PCR and metagenomic sequencing in environmental 1118 samples with these bacteria.

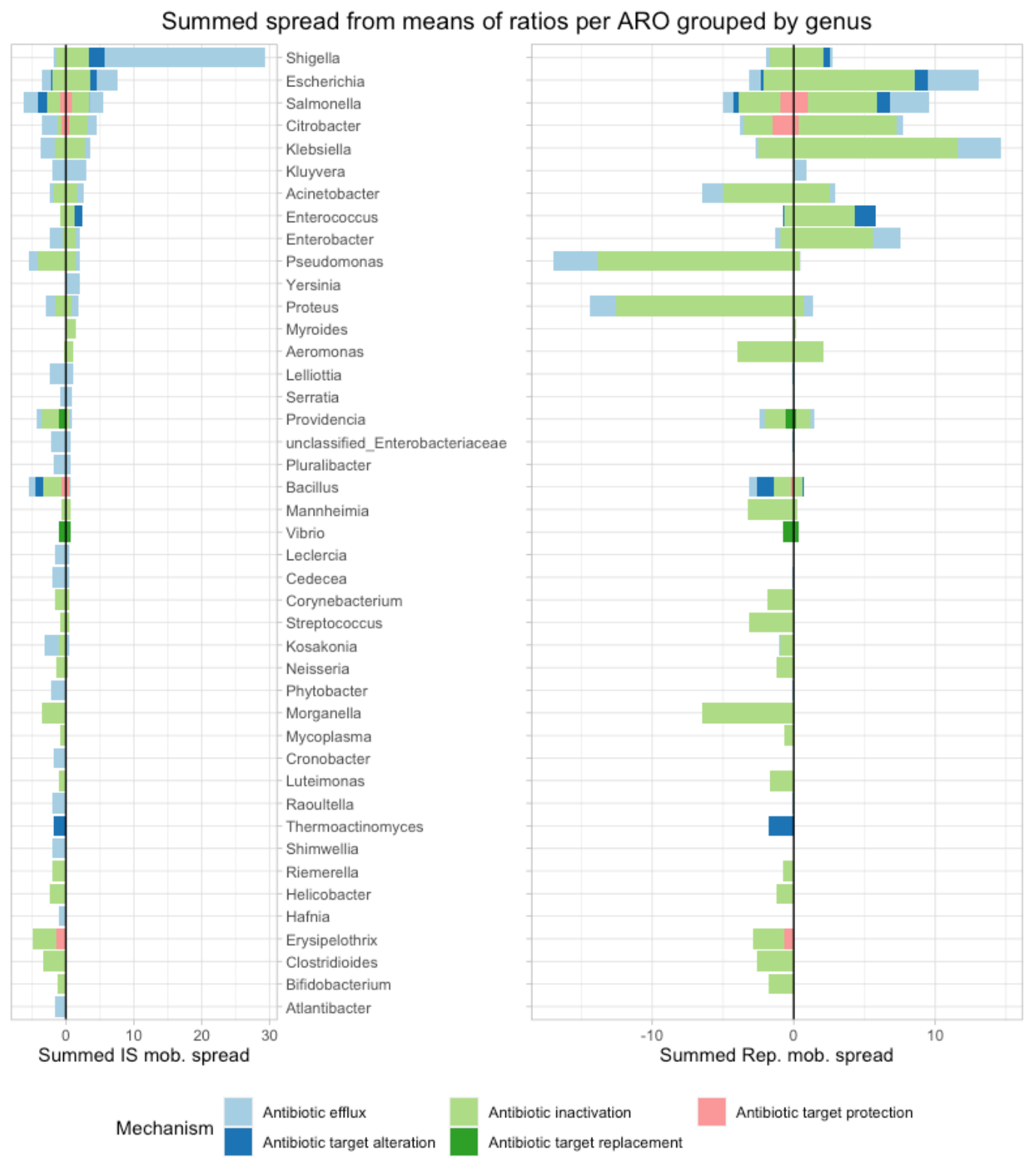

1121 Supplementary Fig. 23: Summed spread from mean IS and Replicon ratios from the ARO means 1122 grouped by genera. The same dataset as for figure Supplementary Fig. 22 is used here, but the 1123 summed spreads are grouped by genera. A large positive summed spread indicates that a given 1124 genus has AROs that are more mobilized, by either IS elements or plasmids, than the ARO mean. 
1126 In Supplementary Table 5, an example of an ARO that is highly differential in IS and plasmid 1127 mobilization, depending on the genus it is found in. The OqxAB efflux pump is encoded by the 1128 neighboring genes $\operatorname{oq} x A$ and $\operatorname{oq} \times B$ that have the same mobilization characteristics. Only results for 1129 oqxA are shown in this example, since oqxB has similar results (not shown). In Klebsiella genomes, 1130 oqxA is found in association with IS elements in $15.5 \%$ of cases and it is only on plasmids in $0.2 \%$. As 1131 such, it should be considered a housekeeping genes, since it does not usually confer resistance 1132 unless highly overexpressed ${ }^{5-7}$.

1134 Supplementary Table 5. Information about the oqxA ARO.

\begin{tabular}{|c|c|c|c|c|c|c|}
\hline Genus & $\begin{array}{l}\text { \#Identified in } \\
\text { total }\end{array}$ & $\begin{array}{l}\text { \# Found on } \\
\text { chromosome }\end{array}$ & $\begin{array}{l}\text { \# Found on } \\
\text { plasmid }\end{array}$ & $\begin{array}{l}\text { \# No IS } \\
\text { associati } \\
\text { on }\end{array}$ & IS Ratio & $\begin{array}{l}\text { Replicon } \\
\text { ratio }\end{array}$ \\
\hline Klebsiella & 458 & 457 & 1 & 387 & 0.155 & 0.002 \\
\hline Enterobacter & 124 & 123 & 1 & 111 & 0.105 & 0.008 \\
\hline Escherichia & 36 & 2 & 34 & 0 & 1.000 & 0.944 \\
\hline Salmonella & 20 & 3 & 17 & 0 & 1.000 & 0.850 \\
\hline Raoultella & 14 & 14 & 0 & 13 & 0.071 & 0.000 \\
\hline Citrobacter & 8 & 8 & 0 & 8 & 0.000 & 0.000 \\
\hline Kosakonia & 7 & 7 & 0 & 4 & 0.429 & 0.000 \\
\hline Lelliottia & 6 & 6 & 0 & 6 & 0.000 & 0.000 \\
\hline Cedecea & 5 & 5 & 0 & 3 & 0.400 & 0.000 \\
\hline Phytobacter & 2 & 2 & 0 & 2 & 0.000 & 0.000 \\
\hline $\begin{array}{l}\text { Unclassified } \\
\text { Enterobacteriaceae }\end{array}$ & 2 & 2 & 0 & 2 & 0.000 & 0.000 \\
\hline
\end{tabular}




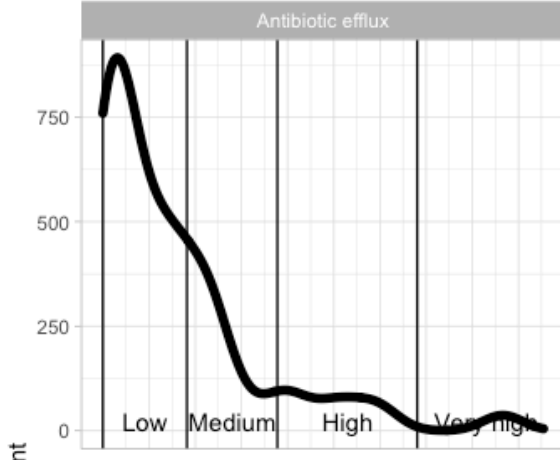

范

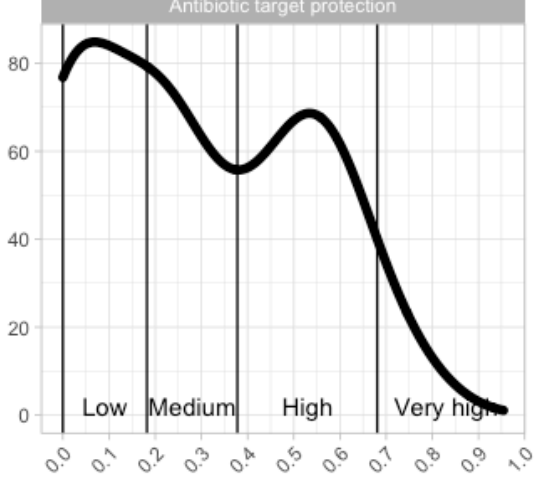

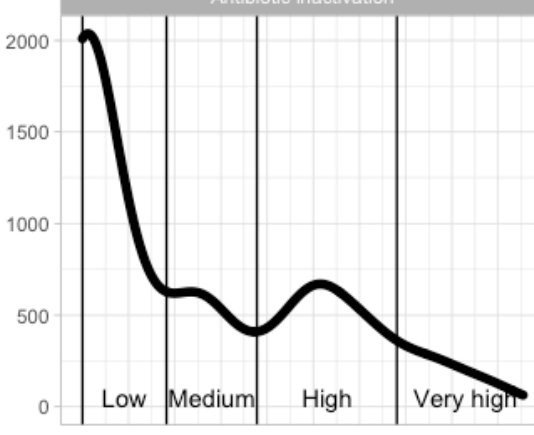

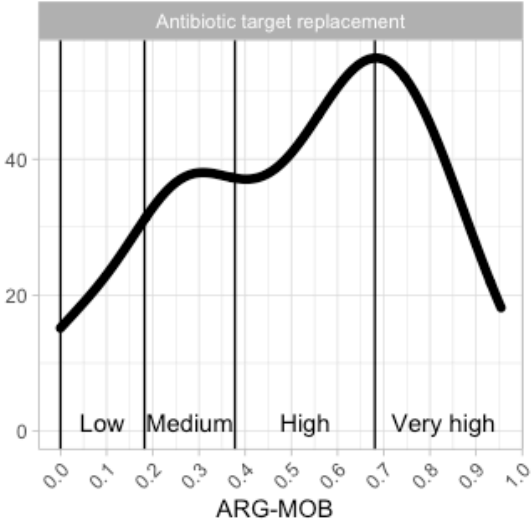

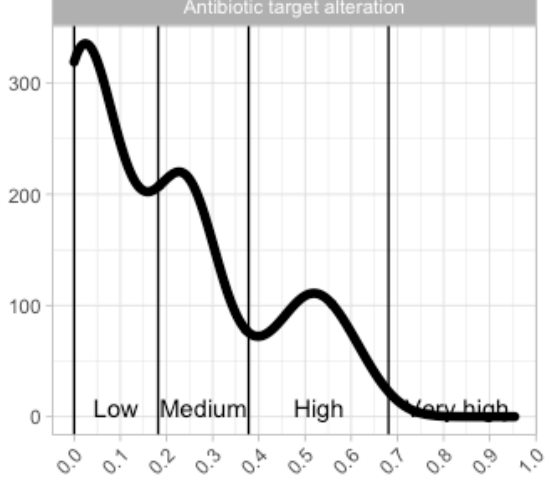

1139 Supplementary Fig. 24: Count density of ARG-MOB per mechanism. Y-axes are not on the same scale 1140 between subplots. The global ARG-MOB category definitions are shown with vertical lines. 
Supplementary Note 11: Examples of Low and High ARG-MOB AROs

\section{Low ARG-MOB examples}

1143 ARG-MOB=0; 3003785 (ATA) MurA transferase: Shown to confer resistance when cloned onto pBAD18 under overexpression in E. coli from Chlamydia ${ }^{8}$

ARG-MOB=0; 3000421 (AE) NorB major facilitator superfamily (MFS) antibiotic efflux pump: Requires overexpression for resistance to quinolones, tetracycline and others. Was cloned from Staphylococci into E. coli for overexpression to give resistance ${ }^{9}$.

ARG-MOB=0; 3000462 (Al) MgtA mgt macrolide glycotransferase resistance: Was shown to confer resistance to macrolides in Streptomyces lividans under overexpression from multicopy plasmid pLST21 with constitutive expression ${ }^{10}$.

ARG-MOB=0; 3004143 ( $A E$ ) resistance-nodulation-cell division (RND) antibiotic efflux pump: The RND efflux pump AxyXY-OprZ is repressed by upstream transcriptional repressor axyZ. Gene deletion mutation of axyz leads to increased axyZ transcription and increased MICs of multiple antibiotics (fluoroquinolones, cefepime, tetracyclines) in Achromobacter ${ }^{11}$. The AxyXY are orthologs of MexX and MexY in Pseudomonas aeruginosa where mutations in the mexZ transcriptional regulator leads overexpression of the MexXY efflux pump and increased resistance ${ }^{12}$. These pumps are likely naturally occurring pumps that require significant overexpression to yield problematic resistance.

ARG-MOB=0; 3004775 (AI) CME beta-lactamase: CME-1 was cloned from a Flavobacterium and overexpressed from a cloning vector in $E$. coli to provide resistance to cefuroxime ${ }^{13}$. The strain was not a clinical isolate.

ARG-MOB=0; 3003035 (ATP) MfpA quinolone resistance protein (qnr): MfpA from Mycobacterium tuberculosis strain H37Rv protects DNA gyrase from fluoroquinolone inhibition. It was found to inherently resistant to fluoroquinolones ${ }^{15}$. 
ARG-MOB=0; 3002646 (Al) APH(3') aminoglycoside phosphotransferase: Aph(3')-Ilc was cloned and overexpressed from the chromosome of Stenotrophomonas maltophilia in E. coli to confer increased MICs to amikacin, butirosin, kanamycin, lividomycin, neomycin, paromomycin, and tobramycin. In this study, Aph(3')-Ilc was knocked out on the chromosome of the WT strain and MICs to butirosin, kanamycin, neomycin and paromomycin were decreased, showing the this gene indeed confers intrinsic resistance those antibiotics. While expression of Aph(3')-Ilc was evaluated with qPCR in the WT strain, the expression levels were not reported and it is thus not known if resistance from this gene is due to mutation in an expression regulator gene in the clinical Stenotrophomonas isolate ${ }^{16}$.

\section{$\underline{\text { High ARG-MOB examples }}$}

ARG-MOB=0.95; 3003013 (ATR) dfrA15 (trimethoprim resistant dihydrofolate reductase dfr): $A$ Class 1 integron with $d f r A 15$ is widespread in Vibrio cholera isolates in Africa, causing resistance to trimethoprim. It was furthermore found on a conjugative plasmid ${ }^{17}$. In this study, it is the ARO with the highest risk, due to the fact that it was only found to be associated with IS elements, integrons and plasmids (all ratios $=1$ ). It has a Simpson index of 0.82 and the 7 CRLs are dispersed across 6 genera (Vibrio, Salmonella, Enterobacter, Leclercia, Klebsiella, and Escherichia).

ARG-MOB=0.91; 3002271 (AI) VIM-1 (VIM beta-lactamase): VIM-1 was originally isolated from a multiresistant E. coli from a patient in Greece. It was inserted in a class 1 integron and found on a conjugative plasmid ${ }^{18}$. It has since been seen in multiple Enterobacteriaceae, typically in association with integrons and plasmids, and is globally spread. Here, it scores the fourth-highest ARG-MOB= of 
ARG-MOB=0.89; 3004635 (Al) $\mathbf{A A C}\left(6^{\prime}\right)$ : During an Enterobacter outbreak in Venezuela, the multiresistance encoding conjugative plasmid pBWH301 was isolated. Amongst other ARGs, aacA7 was 1202 found to encode $A A C\left(6^{\prime}\right)-I$ aminoglycoside acetyltransferase in an integron ${ }^{19}$. In RefSeq complete genomes, there are 12 CRLs which are all on plasmids and associated with IS elements. Furthermore, it is inserted in integrons in $92 \%$ of cases. It is dispersed across 3 genera for a Simpson index of 0.63.

1205 Several other AROs for AAC (6') subtypes are among the highest ARG-MOB scoring AROs, resulting 1206 in one of the highest mean ARG-MOB scores for any Antibiotic Inactivation submechanism ().

ARG-MOB=0.86; 3002847 (Al) rifampin ADP-ribosyltransferase (Arr): From a multi-resistant clinical $P$. aeruginosa isolate, a class I integron was cloned into an expression vector and transformed into E. coli to screen for rifampin resistance ${ }^{20}$. Although an expression vector was used to identify rifampin resistance genes under heterologous expression, it was described that the DNA insert of the clone carrying rifampin resistance gene arr-2 also carried a class I integron with arr-2 inserted as a gene cassette. This corroborates our finding that arr-2 is a high risk putative ARG where it was associated with IS elements in $94 \%$ of CRLs, found on plasmids on $87 \%$, and found as integron gene cassettes in $96 \%$ of CRLs ( $n=54)$. Furthermore, it was found in 8 distinct genera, for a Simpson index of 0.64 (Acinetobacter, Citrobacter, Escherichia, Klebsiella, Proteus, Pseudomonas, Salmonella, and Shewanella). 


\section{Supplementary References}

1219 1. Tansirichaiya, S. The Transposon Registry. Mobile DNA (2019) doi:10.1186/s13100-019-

$1220 \quad$ 0182-3.

$12212 . \quad$ Seemann, T. barrnap 0.9 : rapid ribosomal RNA prediction.

1222 https://github.com/tseemann/barrnap.

1223 3. Gillings, M. R. Genomics and the evolution of antibiotic resistance. Ann. N. Y. Acad. Sci. (2017) doi:10.1111/nyas.13268.

4. $\quad$ Ramirez, M. S. Aminoglycoside modifying enzymes. Drug Resist. Updat. (2010) doi:10.1016/j.drup.2010.08.003.

5. $\mathrm{Li}, \mathrm{J}$. The nature and epidemiology of $\operatorname{OqxAB}$, a multidrug efflux pump. Antimicrobial Resistance and Infection Control (2019) doi:10.1186/s13756-019-0489-3.

6. Perez, F. OqxAB, a quinolone and olaquindox efflux pump, is widely distributed among

7. Yuan, J. Prevalence of the oqxAB gene complex in Klebsiella pneumoniae and Escherichia coli clinical isolates. J. Antimicrob. Chemother. (2012) doi:10.1093/jac/dks086.

8. McCoy, A. J. In vitro and in vivo functional activity of Chlamydia MurA, a UDP-Nacetylglucosamine enolpyruvyl transferase involved in peptidoglycan synthesis and fosfomycin resistance. J. Bacteriol. (2003) doi:10.1128/JB.185.4.1218-1228.2003.

9. Truong-Bolduc, Q. C. MgrA is a multiple regulator of two new efflux pumps in Staphylococcus aureus. J. Bacteriol. (2005) doi:10.1128/JB.187.7.2395-2405.2005.

11. Bador, J. Role of AxyZ transcriptional regulator in overproduction of AxyXY-OprZ multidrug

12. Morita, Y. Primary mechanisms mediating aminoglycoside resistance in the multidrugresistant Pseudomonas aeruginosa clinical isolate PA7. Microbiology (2012) doi:10.1099/mic.0.054320-0.

13. Rossolini, G. M. Cloning of a Chryseobacterium (Flavobacterium) meningosepticum 
chromosomal gene (blaA(CME)) encoding an extended-spectrum class a $\beta$-lactamase related to the Bacteroides cephalosporinases and the VEB-1 and PER $\beta$-lactamases. Antimicrob. Agents Chemother. (1999) doi:10.1128/aac.43.9.2193.

14. Hegde, S. S. Biochemistry: A fluoroquinolone resistance protein from Mycobacterium tuberculosis that mimics DNA. Science (80-. ). (2005) doi:10.1126/science.1110699.

15. Poissy, J. Should moxifloxacin be used for the treatment of extensively drug-resistant tuberculosis? An answer from a murine model. Antimicrob. Agents Chemother. (2010) doi:10.1128/AAC.00968-10.

16. Okazaki, A. Aph( $\left.3^{\prime}\right)-I l c$, an aminoglycoside resistance determinant from Stenotrophomonas maltophilia. Antimicrob. Agents Chemother. (2007) doi:10.1128/AAC.00795-06.

17. Ceccarelli, D. Prevalence of aadA1 and dfrA15 class 1 integron cassettes and SXT circulation in Vibrio cholerae $\mathrm{O} 1$ isolates from Africa. Journal of Antimicrobial Chemotherapy (2006) doi:10.1093/jac/dkl352.

18. Miriagou, V. Escherichia coli with a self-transferable, multiresistant plasmid coding for metallo- $\beta$-lactamase VIM-1. Antimicrob. Agents Chemother. (2003) doi:10.1128/AAC.47.1.395-397.2003.

19. Bunny, K. L. New mobile gene cassettes containing an aminoglycoside resistance gene, aacA7, and a chloramphenicol resistance gene, catB3, in an integron in pBWH301. Antimicrob. Agents Chemother. (1995) doi:10.1128/AAC.39.3.686.

20. Tribuddharat, C. Integron-mediated rifampin resistance in Pseudomonas aeruginosa. 